\title{
Symmetric single-impurity Kondo model on a tight-binding chain: Comparison of analytical and numerical ground-state approaches
}

\author{
Gergely Barcza, ${ }^{1}$ Kevin Bauerbach $\odot,{ }^{2}$ Fabian Eickhoff, ${ }^{3}$ Frithjof B. Anders $\odot,{ }^{3}$ Florian Gebhard $\odot,{ }^{2,}{ }^{*}$ and Örs Legeza ${ }^{1}$ \\ ${ }^{1}$ Strongly Correlated Systems Lendület Research Group, Institute for Solid State Physics and Optics, MTA Wigner Research Centre for \\ Physics, P.O. Box 49, H-1525 Budapest, Hungary \\ ${ }^{2}$ Fachbereich Physik, Philipps-Universität Marburg, D-35032 Marburg, Germany \\ ${ }^{3}$ Theoretische Physik 2, Technische Universität Dortmund, D-44221 Dortmund, Germany
}

(Received 19 November 2019; revised manuscript received 27 January 2020; accepted 27 January 2020; published 24 February 2020)

\begin{abstract}
We analyze the ground-state energy, local spin correlation, impurity spin polarization, impurity-induced magnetization, and corresponding zero-field susceptibilities of the symmetric single-impurity Kondo model on a tight-binding chain with bandwidth $W=2 \mathcal{D}$ where a spin- $\frac{1}{2}$ impurity at the chain center interacts with coupling strength $J_{\mathrm{K}}$ with the local spin of the bath electrons. We compare perturbative results and variational upper bounds from Yosida, Gutzwiller, and first-order Lanczos wave functions to the numerically exact extrapolations obtained from the density-matrix renormalization group (DMRG) method and from the numerical renormalization group (NRG) method performed with respect to the inverse system size and Wilson parameter, respectively. In contrast to the Lanczos and Yosida wave functions, the Gutzwiller variational approach becomes exact in the strong-coupling limit $J_{\mathrm{K}} \gg W$, and reproduces the ground-state properties from DMRG and NRG for large couplings $J_{\mathrm{K}} \gtrsim W$, with a high accuracy. For weak coupling, the Gutzwiller wave function describes a symmetry-broken state with an oriented local moment, in contrast to the exact solution. We calculate the impurity spin polarization and its susceptibility in the presence of magnetic fields that are applied globally or only locally to the impurity spin. The Yosida wave function provides qualitatively correct results in the weak-coupling limit. In DMRG, chains with about $10^{3}$ sites are large enough to describe the susceptibilities down to $J_{\mathrm{K}} / \mathcal{D} \approx 0.6$. For smaller Kondo couplings, only the NRG provides reliable results for a general host-electron density of states $\rho_{0}(\epsilon)$. To compare with results from Bethe ansatz that become exact in the wide-band limit, we study the impurity-induced magnetization and zero-field susceptibility. For small Kondo couplings, the zero-field susceptibilities at zero temperature approach $\chi_{0}\left(J_{\mathrm{K}} \ll \mathcal{D}\right) /\left(g \mu_{\mathrm{B}}\right)^{2} \approx$ $\exp \left[1 /\left(\rho_{0}(0) J_{\mathrm{K}}\right)\right] /\left[2 C \mathcal{D} \sqrt{\pi e \rho_{0}(0) J_{\mathrm{K}}}\right]$, where $\ln (C)$ is the regularized first inverse moment of the density of states. Using NRG, we determine the universal subleading corrections up to second order in $\rho_{0}(0) J_{\mathrm{K}}$.
\end{abstract}

DOI: 10.1103/PhysRevB.101.075132

\section{INTRODUCTION}

\section{A. Kondo problem and Kondo model}

Magnetic moments that couple antiferromagnetically to electron spins of a metallic host pose a difficult many-particle problem because the spin-flip scattering of the host electrons off the impurity spin couples the bath degrees of freedom in an intricate way. Experimentally, this leads to surprising phenomena such as the Kondo resistance minimum around some characteristic low-temperature energy scale $T_{\mathrm{K}}$ [1], often referred to as the Kondo temperature.

Using standard high-temperature perturbation theory to third order in the coupling between the impurity spin and the host electrons, Kondo was able to explain the resistance minimum [2]. However, within standard perturbation theory the resistivity and many other physical quantities like the zero-field magnetic susceptibility diverge logarithmically at

*florian.gebhard@physik.uni-marburg.de zero temperature. A summation of the leading logarithmically diverging terms in the perturbation expansion leads to a divergence at $T_{\mathrm{K}}$ [3,4]. Consequently, approaches beyond perturbation theory are required to describe adequately the ground state of the coupled system of impurity spin and host electrons [4].

This "Kondo problem" inspired the development of scaling concepts that were eventually formalized in Wilson's renormalization group (RG) [5]. Since the Wilson RG can be carried out analytically only to a limited degree, it found its widespread implementation as numerical renormalization group (NRG) method which is best suited for the study of impurity problems [5-7]; for a review, see Ref. [8].

At zero temperature, the impurity spin and the electrons in its surrounding "Kondo cloud" form a "Kondo singlet" as the many-particle ground state; its elementary excitations describe a Fermi liquid [9]. The Bethe ansatz permits the exact solution of the Kondo model with infinite bandwidth (see the reviews by Tsvelick and Wiegmann [10] and by Andrei, Furuya, and Lowenstein [11]). The Bethe ansatz confirms the findings of $(\mathrm{N}) \mathrm{RG}$, and provides analytical formulas, e.g., 
for the impurity magnetization at finite temperatures, and for the Kondo temperature in terms of the Bethe-ansatz parameters. Since NRG provides explicit results also for dynamical quantities at finite temperatures, the Kondo problem could be declared "solved."

The Kondo problem poses one of the fundamental challenges in theoretical many-body physics. Therefore, one might think that the ground-state properties of the Kondo model have been studied in very much detail. Surprisingly, this is not the case. For example, the dependence of the ground-state energy on the Kondo coupling is largely unknown, apart from a study by Mancini and Mattis who used the Lanczos approach [12]. To the best of our knowledge, the large-coupling limit of the Kondo model has not been analyzed extensively yet. Moreover, more elaborate variational states such as the Gutzwiller wave function were not applied to the Kondo model thus far.

It was not until recently that Schnack and Höck used the NRG to investigate the magnetization and zero-field susceptibility for some weak couplings [13]. They emphasized that the impurity spin polarization differs from the impurity-induced magnetization for the whole system, as derivable from the free energy. Moreover, they revived the question how the Bethe-ansatz results can be used for comparison with NRG data because the Kondo couplings in the Bethe ansatz and for a lattice model are related in a nontrivial way. For the series expansion of the Bethe-ansatz coupling $J_{\mathrm{K}}^{\mathrm{BA}}$ in terms of the bare model parameter $J_{\mathrm{K}}$, only the leading-order terms are known analytically from scaling arguments [4] and Wilson's RG [5].

With our work, we fill some of the gaps in the quantitative analysis of the symmetric Kondo model at zero temperature. We study the ground-state energy, the local spin correlation function, the impurity spin polarization and the impurityinduced magnetization as a function of a global and a local magnetic field, and the corresponding zero-field susceptibilities. In the absence of an external field, we perform weakcoupling and strong-coupling perturbation theory. We employ three analytical variational approaches (first-order Lanczos $[12,14,15]$, Yosida [16,17], and Gutzwiller states [18,19]), and perform numerical calculations using the density-matrix renormalization group (DMRG) [20-22] and the numerical renormalization group methods $[5-8,13]$. We compare to Bethe-ansatz $[10,11]$ results where possible.

\section{B. Outline}

Our work is organized as follows. In Sec. II we define the Kondo Hamiltonian on a chain and the ground-state properties that we investigate in the thermodynamic limit, namely, the ground-state energy, local spin correlation function, impurity spin polarization and impurity-induced magnetization, and the corresponding zero-field magnetic susceptibilities.

In Sec. III we employ perturbation theory as first analytical method to derive the ground-state energy and the local spin correlation for weak and strong Kondo couplings. These results provide a benchmark test for all approximate analytical and numerical methods.

Next, in Sec. IV, we derive a variational bound for the ground-state energy from the first-order Lanczos state. As the energy bound is poor, we refrain from calculating magnetic properties for this state.

As a more suitable variational state, we study the Yosida wave function in Sec. V. When properly generalized to finite magnetic fields, it permits the analytic calculation of magnetic ground-state properties in the presence of a local and a global magnetic field. Although the Yosida state gives a poor estimate for the ground-state energy, it provides a qualitatively correct description of the zero-field magnetic susceptibilities at small Kondo couplings.

As a third analytic variational approach, we study the Gutzwiller wave function in Sec. VI. It can be viewed as a Hartree-Fock ground state for the Kondo model where the condition of a spin on the impurity is guaranteed. From the Hartree-Fock perspective, it is not too surprising that the Gutzwiller state contains an artificial transition from a phase with a broken local symmetry at small Kondo couplings to a phase with a local spin singlet at large Kondo couplings. Apart from this flaw, the ground-state energy and the local spin correlation are in very good agreement with numerically exact data from NRG and DMRG. The Gutzwiller state becomes exact for strong couplings.

As the last analytic approach, we recall results from the Bethe ansatz in Sec. VII. The Bethe ansatz solves a related Kondo model that has a linear dispersion relation with an infinite bandwidth so that it is a nontrivial task to establish the link to the parameters in the lattice model. This is accomplished by Wilson's renormalization group, and we use perturbation theory to calculate analytically the leading-order terms for the zero-field impurity-induced susceptibility.

In Sec. VIII we discuss two numerically exact approaches to the Kondo problem, namely, the numerical renormalization group (NRG) and the density-matrix renormalization group (DMRG) methods. The DMRG treats finite chains with up to $L \approx 1000$ sites with a very high numerical accuracy. Thereby, DMRG provides excellent variational upper bounds for the ground-state energy and the local spin correlation function. Since it has an essentially constant energy resolution over the whole band, our present version of DMRG cannot access the exponentially small Kondo scale that develops for small Kondo couplings. The NRG was developed and designed to treat these Kondo scales and therefore provides access to small Kondo couplings as well.

In Sec. IX we compare the results of all methods. The Gutzwiller approach provides the best analytic variational state for the ground-state energy and the local spin correlation function. The Gutzwiller wave function becomes the exact ground state for large Kondo couplings, and reliably describes the physics when the Kondo coupling becomes larger than the host-electron bandwidth. The DMRG provides excellent values for the ground-state properties, and our analysis of the finite-size data only fails to describe magnetic properties when the Kondo energy scale becomes exponentially small. The NRG is found to work very well for all cases. In particular, it permits to determine the different subleading terms of the zero-field magnetic susceptibilities when they become exponentially large as a function of the Kondo coupling.

In Sec. X, we summarize and briefly discuss our findings. We defer technical details to Appendix A and provide 
extensive calculations in the Supplemental Material [23], as listed in Appendix B.

\section{SINGLE-IMPURITY KONDO MODEL ON A CHAIN}

We start our investigation with the definition of the model Hamiltonian. Next, we list the ground-state quantities that we study in this work.

\section{A. Hamiltonian of the single-impurity Kondo model}

In the strong-coupling limit, a Schrieffer-Wolff transformation maps the symmetric single-impurity Anderson model (SIAM) to the the $s-d$ (or single-impurity Kondo) model (SIKM) $[4,24,25]$

$$
\hat{H}_{\mathrm{K}}=\hat{T}+\hat{V}_{\mathrm{sd}}+\hat{H}_{\mathrm{m}} .
$$

We consider a chain with an odd number of sites $L, n=$ $-(L-1) / 2, \ldots,(L-1) / 2$, and we choose $L$ such that $(L+$ $3) / 2$ is even. The operator for the kinetic energy of the conduction electrons reads as

$$
\hat{T}=-t \sum_{n=-(L-1) / 2, \sigma}^{(L-3) / 2}\left(\hat{c}_{n, \sigma}^{+} \hat{c}_{n+1, \sigma}+\hat{c}_{n+1, \sigma}^{+} \hat{c}_{n, \sigma}\right)
$$

In the absence of an external magnetic field, we address a paramagnetic half-filled system $N_{\uparrow}=N_{\downarrow}=(L+1) / 2$.

The impurity couples purely locally at the center of the chain. For a local hybridization in the symmetric SIAM and for strong coupling, the Kondo coupling becomes

$$
\begin{aligned}
\hat{V}_{\mathrm{sd}}= & J_{\mathrm{K}} \mathbf{s}_{0} \cdot \mathbf{S}, \\
\mathbf{s}_{0} \cdot \mathbf{S}= & \frac{1}{2}\left(\hat{c}_{0, \uparrow}^{+} \hat{c}_{0, \downarrow} \hat{d}_{\downarrow}^{+} \hat{d}_{\uparrow}+\hat{c}_{0, \downarrow}^{+} \hat{c}_{0, \uparrow} \hat{d}_{\uparrow}^{+} \hat{d}_{\downarrow}\right) \\
& +\frac{1}{4}\left(\hat{d}_{\uparrow}^{+} \hat{d}_{\uparrow}-\hat{d}_{\downarrow}^{+} \hat{d}_{\downarrow}\right)\left(\hat{c}_{0, \uparrow}^{+} \hat{c}_{0, \uparrow}-\hat{c}_{0, \downarrow}^{+} \hat{c}_{0, \downarrow}\right) .
\end{aligned}
$$

The host electron spin $\mathbf{s}_{0}$ at site $n=0$ interacts locally with the impurity spin $\mathbf{S}$ with coupling strength $J_{\mathrm{K}} \geqslant 0$. Note that in Eq. (3) it is implicitly understood that $\hat{H}_{\mathrm{K}}$ only acts in the subspace of singly occupied $d$ levels.

To study the magnetization and magnetic susceptibility, we add an external magnetic field $\mathcal{H}>0$,

$$
\hat{H}_{\mathrm{m}}=-B\left(\hat{d}_{\uparrow}^{+} \hat{d}_{\uparrow}-\hat{d}_{\downarrow}^{+} \hat{d}_{\downarrow}+\sum_{n=-(L-1) / 2}^{(L-1) / 2} \hat{c}_{n, \uparrow}^{+} \hat{c}_{n, \uparrow}-\hat{c}_{n, \downarrow}^{+} \hat{\iota}_{n, \downarrow}\right),
$$

where we denote the magnetic energy by

$$
B=g_{e} \mu_{\mathrm{B}} \mathcal{H} / 2>0,
$$

$g_{e} \approx 2$ is the electronic gyromagnetic factor, and $\mu_{\mathrm{B}}$ is the Bohr magneton. For completeness, we shall also consider the case where the magnetic field is applied only at the impurity site

$$
\hat{H}_{\mathrm{m}, \mathrm{loc}}=-B\left(\hat{d}_{\uparrow}^{+} \hat{d}_{\uparrow}-\hat{d}_{\downarrow}^{+} \hat{d}_{\downarrow}\right) .
$$

The kinetic energy of the host electrons is diagonal in momentum space (see Appendix A 1)

$$
\hat{T}=\sum_{k=1, \sigma}^{L} \epsilon_{k} \hat{a}_{k, \sigma}^{+} \hat{a}_{k, \sigma}
$$

with the dispersion relation $\epsilon_{k}$. The corresponding density of states is defined by

$$
\rho_{0}(\omega)=\frac{1}{L} \sum_{k=1}^{L} \delta\left(\omega-\epsilon_{k}\right)=\frac{1}{\pi} \frac{1}{\sqrt{(2 t)^{2}-\omega^{2}}}
$$

for $|\omega|<2 t$. We use half the bandwidth as our unit of energy, $2 t \equiv 1, W \equiv 2$, to make a direct contact with the Bethe-ansatz calculations. In numerical DMRG calculations, the model is mapped onto a half-chain with the impurity at the left chain end. This is done in Appendix A 2.

For some of our analytic calculations, we shall treat $\rho_{0}(\omega)$ as a selectable quantity. In all cases, the density of states is supposed to be regular at the Fermi energy $0<\rho_{0}(0)<$ $\infty$. This rules out, e.g., a tight-binding model for the host electrons in two dimensions with its logarithmic van Hove singularity at the Fermi energy. Note that, for $J_{\mathrm{K}} \rightarrow 0$, it is mostly $\rho_{0}(0)$ that governs the physics of the SIKM. Therefore, the results for a one-dimensional or a constant density of states are representative for the symmetric SIKM at weak coupling.

\section{B. Ground-state properties}

In this work, we are interested in the excess ground-state energy due to the presence of the coupled impurity spin, the local spin correlation the impurity spin polarization for a global and a local field, and the corresponding susceptibilities. Moreover, for comparison with Bethe ansatz, we also address the impurity-induced magnetization and zero-field susceptibility for global and local fields.

\section{Ground-state energy and local spin correlation}

We calculate the excess ground-state energy due to the presence of the impurity, i.e., the impurity-induced change of the ground-state energy of free electrons,

$$
e_{0}\left(J_{\mathrm{K}}, L\right)=E_{0}\left(J_{\mathrm{K}}, L\right)-E_{\mathrm{FS}}(L) .
$$

The impurity-induced energy contribution $e_{0}\left(J_{\mathrm{K}}, L\right)$ is of order unity and $e_{0}\left(J_{\mathrm{K}}=0, L\right)=0$. Eventually, we extrapolate to the thermodynamic limit

$$
e_{0}\left(J_{\mathrm{K}}\right)=\lim _{L \rightarrow \infty} e_{0}\left(J_{\mathrm{K}}, L\right) .
$$

This is done explicitly using DMRG. In contrast, the NRG discretizes the continuum model in energy space (see Sec. VIII). The analytic calculations are directly performed in the thermodynamic limit.

Another quantity of interest is the local spin correlation function in the ground state

$$
C_{0}^{S}\left(J_{\mathrm{K}}\right)=\left\langle\mathbf{s}_{0} \cdot \mathbf{S}\right\rangle .
$$

It can either be calculated directly, or from the HellmannFeynman theorem (see Appendix B 1) [26,27]:

$$
C_{0}^{S}\left(J_{\mathrm{K}}\right)=\frac{\partial e_{0}\left(J_{\mathrm{K}}\right)}{\partial J_{\mathrm{K}}} .
$$

In turn, we may calculate the ground-state energy from the local spin correlation using

$$
e_{0}\left(J_{\mathrm{K}}\right)=\int_{0}^{J_{\mathrm{K}}} d J C_{0}^{S}(J) .
$$


Therefore, Eq. (13) can be used to check the consistency of the ground-state calculations because Eqs. (12) and (13) hold for the exact ground state. Note that the HellmannFeynman theorem also applies to variational approaches (see Appendix B 1).

\section{Ground-state impurity spin polarization, impurity-induced magnetization, and zero-field susceptibilities}

a. Global external field. In the presence of an external magnetic field $\mathcal{H}$, the spin on the impurity orients itself so that the spin projection into the direction of the external field becomes finite. We denote the impurity spin polarization as

$$
\begin{aligned}
m^{S}\left(J_{\mathrm{K}}, B\right) & =g_{\mathrm{e}} \mu_{\mathrm{B}} S^{z}\left(J_{\mathrm{K}}, B\right), \\
S^{z}\left(J_{\mathrm{K}}, B\right) & =\left\langle\hat{S}^{z}\right\rangle=\frac{1}{2}\left\langle\hat{n}_{d, \uparrow}-\hat{n}_{d, \downarrow}\right\rangle .
\end{aligned}
$$

Correspondingly, we define the impurity spin susceptibility via the relation

$$
\chi^{S}\left(J_{\mathrm{K}}, B\right)=\frac{\partial m^{S}\left(J_{\mathrm{K}}, B\right)}{\partial \mathcal{H}}=\frac{g_{e} \mu_{\mathrm{B}}}{2} \frac{\partial m^{S}\left(J_{\mathrm{K}}, B\right)}{\partial B} .
$$

The impurity spin polarization and susceptibility can straightforwardly be calculated for our various ground-state approaches.

The impurity spin polarization must not be confused with the thermodynamic magnetization of the system,

$$
m\left(J_{\mathrm{K}}, B, T\right)=-\frac{\partial \mathcal{F}\left(J_{\mathrm{K}}, B, T\right)}{\partial \mathcal{H}}=g_{e} \mu_{\mathrm{B}} S_{\mathrm{tot}}^{z}\left(J_{\mathrm{K}}, B, T\right),
$$

where $T=1 / \beta$ is the temperature, and the total spin projection in the direction of the external field is

$$
\begin{aligned}
S_{\mathrm{tot}}^{z}\left(J_{\mathrm{K}}, B, T\right) & =S^{z}\left(J_{\mathrm{K}}, B, T\right)+s^{z}\left(J_{\mathrm{K}}, B, T\right), \\
S^{z}\left(J_{\mathrm{K}}, B, T\right) & =\left\langle\hat{S}^{z}\right\rangle=\frac{1}{2}\left\langle\hat{n}_{d, \uparrow}-\hat{n}_{d, \downarrow}\right\rangle, \\
s^{z}\left(J_{\mathrm{K}}, B, T\right) & =\left\langle\hat{s}^{z}\right\rangle=\sum_{n=-(L-1) / 2}^{(L-1) / 2} \frac{\left\langle\hat{c}_{n, \uparrow}^{+} \hat{c}_{n, \uparrow}-\hat{c}_{n, \downarrow}^{+} \hat{c}_{n, \downarrow}\right\rangle}{2},
\end{aligned}
$$

where the angular brackets imply the thermal average.

Since $s^{z}\left(J_{\mathrm{K}}, B, T\right)$ is proportional to the system size, the thermodynamic magnetization is not a useful quantity because the impurity spin contribution $S^{z}\left(J_{\mathrm{K}}, B, T\right)$ is only of order unity. Therefore, it is more sensible to define impurityinduced changes to thermodynamic quantities due to the presence of the impurity $[10,11,13]$. The impurity-induced free energy is defined by

$$
\begin{aligned}
\mathcal{F}^{\mathrm{ii}}\left(J_{\mathrm{K}}, B, T\right)= & -T \ln \operatorname{Tr}\left[\exp \left(-\beta \hat{H}_{\mathrm{K}}\right)\right] \\
& +T \ln \operatorname{Tr}\left[\exp \left(-\beta\left(\hat{T}+\hat{H}_{\mathrm{m}}\right)\right)\right],
\end{aligned}
$$

where the chemical potential is $\mu(T)=0$ for the particlehole-symmetric Kondo and free-fermion Hamiltonians at all temperatures [28]. The derivative with respect to $\mathcal{H}$ gives the impurity-induced magnetization

$$
m^{\mathrm{ii}}\left(J_{\mathrm{K}}, B, T\right)=g_{e} \mu_{\mathrm{B}}\left(S_{\mathrm{tot}}^{z}\left(J_{\mathrm{K}}, B, T\right)-s^{z, \text { free }}(B, T)\right) .
$$

It is of the order unity.

In Eq. (18) we have $\mathcal{F}^{\mathrm{ii}}\left(J_{\mathrm{K}}, B, T=0\right)=e_{0}\left(J_{\mathrm{K}}, B\right)$ at zero temperature so that we can obtain the impurity-induced magnetization also from the excess ground-state energy

$$
m^{\mathrm{ii}}\left(J_{\mathrm{K}}, B\right)=-\frac{g_{e} \mu_{\mathrm{B}}}{2} \frac{\partial e_{0}\left(J_{\mathrm{K}}, B\right)}{\partial B} .
$$

The impurity-induced magnetic susceptibility at zero temperature follows as

$$
\begin{aligned}
\chi^{\mathrm{ii}\left(J_{\mathrm{K}}, B\right)} & =\frac{g_{e} \mu_{\mathrm{B}}}{2} \frac{\partial m^{\mathrm{ii}}\left(J_{\mathrm{K}}, B\right)}{\partial B} \\
& =-\left(\frac{g_{e} \mu_{\mathrm{B}}}{2}\right)^{2} \frac{\partial^{2} e_{0}\left(J_{\mathrm{K}}, B\right)}{\partial B^{2}} .
\end{aligned}
$$

We abbreviate the impurity-induced susceptibility at zero field as $\chi_{0}^{\mathrm{ii}}\left(J_{\mathrm{K}}\right) \equiv \chi^{\mathrm{ii}}\left(J_{\mathrm{K}}, B=0\right)$.

b. Local external field. When the magnetic field is applied only at the impurity site, we denote the corresponding quantities by an extra lower index "loc," e.g., $m_{\mathrm{loc}}^{S}\left(J_{\mathrm{K}}, B\right)$ and $\chi_{\mathrm{loc}}^{S}\left(J_{\mathrm{K}}, B\right)$. For a local field, the impurity spin polarization and susceptibility are the proper thermodynamic quantities. They can be calculated from the ground-state energy in the presence of a local field:

$$
\begin{aligned}
m_{\mathrm{loc}}^{S}\left(J_{\mathrm{K}}, B\right) & =-\frac{g_{e} \mu_{\mathrm{B}}}{2} \frac{\partial e_{0, \mathrm{loc}}\left(J_{\mathrm{K}}, B\right)}{\partial B}, \\
\chi_{\mathrm{loc}}^{S}\left(J_{\mathrm{K}}, B\right) & =\frac{g_{e} \mu_{\mathrm{B}}}{2} \frac{\partial m_{\mathrm{loc}}^{S}\left(J_{\mathrm{K}}, B\right)}{\partial B} \\
& =-\left(\frac{g_{e} \mu_{\mathrm{B}}}{2}\right)^{2} \frac{\partial^{2} e_{0, \mathrm{loc}}\left(J_{\mathrm{K}}, B\right)}{\partial B^{2}} .
\end{aligned}
$$

For a local field, the free host electron system is unpolarized. Therefore, the impurity-induced magnetization in the presence of a local field describes the impurity spin polarization plus the induced magnetization of the host electrons and thus is of order unity,

$$
\begin{aligned}
m_{\mathrm{loc}}^{\mathrm{ii}}\left(J_{\mathrm{K}}, B\right) & =g_{e} \mu_{\mathrm{B}} S_{\mathrm{tot}}^{z}\left(J_{\mathrm{K}}, B\right) \\
& =g_{e} \mu_{\mathrm{B}}\left(S^{z}\left(J_{\mathrm{K}}, B\right)+s^{z}\left(J_{\mathrm{K}}, B\right)\right)
\end{aligned}
$$

at zero temperature. In general, the impurity-induced magnetization is smaller than the impurity spin polarization because it is reduced by the contribution of the bath electron screening cloud $s^{z}\left(J_{\mathrm{K}}, B\right)<0$.

c. Zero-field susceptibilities. There are four different susceptibilities at finite fields but only three different zero-field susceptibilities because

$$
\chi_{0}^{S}\left(J_{\mathrm{K}}, T\right)=\chi_{0, \text { loc }}^{\mathrm{ii}}\left(J_{\mathrm{K}}, T\right)
$$

holds for all temperatures. To see this, we recall the definition of the impurity-induced magnetization at finite local field $B$,

$$
\begin{aligned}
\frac{m_{\mathrm{loc}}^{\mathrm{ii}}\left(J_{\mathrm{K}}, B, T\right)}{g_{e} \mu_{\mathrm{B}}} & =\left\langle\hat{S}^{z}+\hat{S}^{z}\right\rangle \\
& =\frac{1}{\mathcal{Z}} \operatorname{Tr}\left[e^{-\beta\left(\hat{T}+\hat{V}_{\mathrm{sd}}-2 B \hat{S}^{z}\right)}\left(\hat{S}^{z}+\hat{s}^{z}\right)\right],
\end{aligned}
$$

so that from Eq. (19) we find

$$
\frac{\chi_{0, \text { loc }}^{\mathrm{ii}}\left(J_{\mathrm{K}}, T\right)}{\left(g_{e} \mu_{\mathrm{B}}\right)^{2}}=\frac{1}{T}\left\langle\hat{S}^{z}\left(\hat{S}^{z}+\hat{S}^{z}\right)\right\rangle,
$$

where we used that the system is unpolarized for $B=0$. 
On the other hand, the impurity spin polarization at finite global field $B$ is defined by

$$
\begin{aligned}
\frac{m^{S}\left(J_{\mathrm{K}}, B, T\right)}{g_{e} \mu_{\mathrm{B}}} & =\left\langle\hat{S}^{z}\right\rangle \\
& =\frac{1}{\mathcal{Z}} \operatorname{Tr}\left[e^{-\beta\left(\hat{T}+\hat{V}_{\mathrm{sd}}-2 B \hat{S}^{z}-2 B \hat{S}^{z}\right)} \hat{S}^{z}\right],
\end{aligned}
$$

so that from Eq. (15) we find

$$
\frac{\chi_{0}^{S}\left(J_{\mathrm{K}}, T\right)}{\left(g_{e} \mu_{\mathrm{B}}\right)^{2}}=\frac{1}{T}\left\langle\left(\hat{S}^{z}+\hat{s}^{z}\right) \hat{S}^{z}\right\rangle,
$$

where we used that the system is unpolarized for $B=0$. A comparison of Eqs. (26) and (28) proves Eq. (24).

Note that the equivalence (24) does not necessarily hold for approximate approaches. In Sec. V we shall see that it is not fulfilled for the Yosida variational approach. As shown in Sec. VI, it is obeyed in the paramagnetic Gutzwiller wave function. For the NRG, Eq. (24) provides a convenient tool to assess the accuracy of the numerical calculations.

\section{PERTURBATION THEORY FOR THE GROUND-STATE ENERGY}

In this section, we derive the excess ground-state energy and local spin correlation function from weak-coupling and strong-coupling perturbation theory at zero magnetic field.

\section{A. Weak-coupling perturbation theory}

When we ignore the coupling between the impurity spin and the bath electrons, the ground state is doubly degenerate. Since we are interested in the ground state, we work with the spin-singlet state

$$
\begin{aligned}
\left|\Phi_{0}\right\rangle & =\sqrt{\frac{1}{2}}\left(\hat{d}_{\downarrow}^{+} \hat{a}_{k_{\mathrm{F}} \downarrow}+\hat{d}_{\uparrow}^{+} \hat{a}_{k_{\mathrm{F}}, \uparrow}\right)|\mathrm{FS}\rangle\left|\mathrm{vac}_{d}\right\rangle \\
& \equiv \sqrt{\frac{1}{2}}(|A\rangle+|B\rangle),
\end{aligned}
$$

where $k_{\mathrm{F}}=(L+1) / 2$ is the Fermi number in the full chain. The state $\left|\Phi_{0}\right\rangle$ is normalized to unity. The ground state of the Kondo Hamiltonian for $J_{\mathrm{K}}=0$ and an empty $d$ level is given by the Fermi sea

$$
|\mathrm{FS}\rangle=\prod_{\sigma}\left|\mathrm{FS}_{\sigma}\right\rangle, \quad\left|\mathrm{FS}_{\sigma}\right\rangle=\prod_{k=1}^{k_{\mathrm{F}}} \hat{a}_{k, \sigma}^{+}|\mathrm{vac}\rangle .
$$

The calculations from standard perturbation theory are carried out in Appendix A 3.

In the thermodynamic limit, there is no first-order correction, and the excess ground-state to second order reads as

$$
e_{0}^{(2)}\left(J_{\mathrm{K}}\right)=-f b_{1}^{2}
$$

with

$$
\begin{gathered}
f=-4 \int_{-1}^{0} d \omega_{1} \int_{-1}^{0} d \omega_{2} \rho_{0}\left(\omega_{1}\right) \rho_{0}\left(\omega_{2}\right) \frac{1}{\omega_{1}+\omega_{2}}, \\
b_{1}^{2}=\left\langle\Phi_{0}\left|\hat{V}_{\mathrm{sd}}^{2}\right| \Phi_{0}\right\rangle .
\end{gathered}
$$

As shown in Appendix B 2, we have $b_{1}^{2}=3 J_{\mathrm{K}}^{2} / 32$, independent of the density of states. In one dimension, $f^{d=1}=1$ (see
Appendix A 3), so that our final result to second order is

$$
e_{0}^{(2)}\left(J_{\mathrm{K}}\right)=-\frac{3}{32} J_{\mathrm{K}}^{2}
$$

for the one-dimensional density of states (8).

For the local spin correlation function we thus find

$$
C_{0}^{S}\left(J_{\mathrm{K}} \ll 1\right)=-\frac{3}{16} J_{\mathrm{K}}+\mathcal{O}\left(J_{\mathrm{K}}^{2}\right)
$$

in the weak-coupling limit.

\section{B. Strong-coupling perturbation theory \\ 1. Leading order}

To leading order in $J_{\mathrm{K}}$, the impurity spin and the electron spin at the origin form a spin singlet. Since

$$
\mathbf{s}_{0} \cdot \mathbf{S}=\frac{1}{2}\left(\left(\mathbf{s}_{0}+\mathbf{S}\right)^{2}-\mathbf{s}_{0}^{2}-\mathbf{S}^{2}\right)=\frac{1}{2}\left(\mathbf{S}_{\mathrm{tot}}^{2}-\mathbf{s}_{0}^{2}-\mathbf{S}^{2}\right)
$$

and $S_{\text {tot }}=0, S=s_{0}=\frac{1}{2}$, we have

$$
e_{0}\left(J_{\mathrm{K}}\right)=-\frac{3 J_{\mathrm{K}}}{4}
$$

to leading order in $J_{\mathrm{K}}$.

\section{Next-to-leading order}

To obtain the correction to order $\left(J_{\mathrm{K}}\right)^{0}$, we realize that the host electrons experience a scattering center at the origin of infinite strengths. As shown in Appendix B 3, in the presence of a local impurity potential of strength $V$, spinless fermions experience the energy shift

$$
e_{0}^{\mathrm{ps}}(V)=-\frac{1}{\pi} \int_{-1-\eta}^{0} d \omega \omega \frac{\partial}{\partial \omega} \operatorname{Cot}^{-1}\left[\frac{1-V \Lambda_{0}(\omega)}{\pi V \rho_{0}(\omega)}\right],
$$

where $\rho_{0}(\omega)$ is the density of states of the free host electrons and $\Lambda_{0}(\omega)$ is its Hilbert transform

$$
\Lambda_{0}(\omega)=f_{-1}^{1} d \epsilon \frac{\rho_{0}(\epsilon)}{\omega-\epsilon} .
$$

Moreover, $\operatorname{Cot}^{-1}(x)=\cot ^{-1}(x)+\pi \theta_{\mathrm{H}}(-x)$ is continuous and differentiable across $x=0$, where $\theta_{\mathrm{H}}(x)$ is the Heaviside step function.

In one dimension, we obtain from Appendix B 3

$$
e_{0}^{\mathrm{ps}}(V)=\frac{1}{2}\left(1+V-\sqrt{1+V^{2}}\right)
$$

for the energy shift per spin species which reduces to

$$
e_{0}^{\mathrm{ps}}(V \rightarrow \infty)=\frac{1}{2}
$$

for $V \rightarrow \infty$. Summing over both spin species we obtain

$$
e_{0}\left(J_{\mathrm{K}}\right)=-\frac{3 J_{\mathrm{K}}}{4}+1
$$

for the strong-coupling limit of the Kondo model, with corrections of the order $\left(J_{\mathrm{K}}\right)^{-1}$.

For the local spin correlation function we thus find

$$
C_{0}^{S}\left(J_{\mathrm{K}} \gg 1\right)=-\frac{3}{4}+\mathcal{O}\left(J_{\mathrm{K}}^{-2}\right)
$$

in the strong-coupling limit. 


\section{LANCZOS VARIATIONAL APPROACH}

As a first variational approach, we consider the Lanczos theory and compile the results for the first-order Lanczos state. The calculations of higher orders quickly become cumbersome and prone to errors. Since the Yosida and Gutzwiller variational description are superior to the Lanczos approach, we only consider the Kondo model without an external magnetic field.

\section{A. Recursive construction}

The Lanczos approach starts from some initial state $\left|\Phi_{0}\right\rangle$, e.g., the state defined in Eq. (29). The next states are constructed recursively $[14,15]$,

$$
\left|\Phi_{n+1}\right\rangle=\hat{H}\left|\Phi_{n}\right\rangle-a_{n}\left|\Phi_{n}\right\rangle-b_{n}^{2}\left|\Phi_{n-1}\right\rangle, \quad n \geqslant 0
$$

where we set $b_{0} \equiv 0$, and

$$
\begin{aligned}
a_{n} & =\frac{\left\langle\Phi_{n}|\hat{H}| \Phi_{n}\right\rangle}{\left\langle\Phi_{n} \mid \Phi_{n}\right\rangle}, \\
b_{n}^{2} & =\frac{\left\langle\Phi_{n} \mid \Phi_{n}\right\rangle}{\left\langle\Phi_{n-1} \mid \Phi_{n-1}\right\rangle} \geqslant 0 .
\end{aligned}
$$

The states $\left|\Phi_{n}\right\rangle$ are not normalized to unity but they are orthogonal to each other (see Appendix B 2).

The real parameters $a_{l}, b_{l}>0$ define the elements of the $(M+1) \times(M+1)$ tridiagonal Hamilton matrix $\underline{\underline{H}}^{(M)}$ with the entries

$$
H_{l, m}=\delta_{l, m+1} b_{l}+\delta_{l, m} a_{l}+\delta_{l, m-1} b_{l+1}
$$

for $0 \leqslant l, m \leqslant M$. Its lowest eigenvalue $\Xi_{0}^{(M)}$ provides a variational upper bound to the ground-state energy $[14,15]$

$$
E_{0} \leqslant \Xi_{0}^{(M)} \leqslant \Xi_{0}^{(M-1)}
$$

for all $M \geqslant 1$. For completeness, we include a simple proof in Appendix B 2.

\section{B. Results for the first-order Lanczos state}

The variational Lanczos energy to leading order is

$$
\Xi_{0}^{(0)}=a_{0}=0
$$

[see Eq. (A21)]. The variational Lanczos energy to first order reads as

$$
\Xi_{0}^{(1)}=\frac{1}{2}\left[a_{1}-\sqrt{a_{1}^{2}+4 b_{1}^{2}}\right] .
$$

The matrix elements in one spatial dimension are calculated in Appendix B 2 with the result

$$
\Xi_{0}^{(1)}\left(J_{\mathrm{K}}\right)=\frac{1}{2}\left[-\frac{J_{\mathrm{K}}}{2}+\frac{4}{\pi}-\sqrt{\left(-\frac{J_{\mathrm{K}}}{2}+\frac{4}{\pi}\right)^{2}+\frac{3 J_{\mathrm{K}}^{2}}{8}}\right] .
$$

To second order in $J_{\mathrm{K}}$, the first-order Lanczos energy reads as

$$
\Xi_{0}^{(1)}\left(J_{\mathrm{K}} \ll 1\right)=-\frac{\pi}{4} \frac{3 J_{\mathrm{K}}^{2}}{32}+\mathcal{O}\left(J_{\mathrm{K}}^{3}\right) .
$$

In comparison with second-order perturbation theory [Eq. (34)], the Lanczos state accounts for $\pi / 4 \approx 78.5 \%$ of the exact second-order term.
For strong coupling, the first-order Lanczos state provides the bound

$$
\begin{aligned}
\Xi_{0}^{(1)}\left(J_{\mathrm{K}} \gg 1\right) & =\frac{1}{8}(-2-\sqrt{10}) J_{\mathrm{K}}+2 \frac{5+\sqrt{10}}{5 \pi} \\
& =-0.645 J_{\mathrm{K}}+1.04 .
\end{aligned}
$$

For $J_{\mathrm{K}} \gg 1$, the first-order Lanczos energy accounts for $86.0 \%$ of the exact ground-state energy given in Eq. (42).

\section{YOSIDA WAVE FUNCTION}

As the next variational theory, we study the Yosida variational state that we generalize to the case of a finite external field. The Yosida state gives a poor variational energy but recovers the exponentially large magnetic susceptibility for small Kondo couplings. Moreover, the calculations can be carried out analytically to a far degree.

\section{A. Yosida variational state}

\section{Definition}

Yosida $[16,17]$ extended $\left|\Phi_{0}\right\rangle$ in Eq. (29) in a generic way, and proposed the variational wave function

$$
\left|\Psi_{\mathrm{Y}}\right\rangle=\sqrt{\frac{1}{2 L}} \sum_{k, \epsilon_{k}>0} \alpha_{k}\left(\hat{a}_{k, \downarrow}^{+} \hat{d}_{\uparrow}^{+}-\hat{a}_{k, \uparrow}^{+} \hat{d}_{\downarrow}^{+}\right)|\mathrm{FS}\rangle\left|\operatorname{vac}_{d}\right\rangle .
$$

Here, $\alpha_{k}$ is real and of the order unity. Note that $\left|\Psi_{\mathrm{Y}}\right\rangle$ is a spin-singlet state.

To include a spin anisotropy at finite external field $B \geqslant 0$, we generalize the Yosida wave function

$$
\begin{aligned}
\left|\Psi_{\mathrm{Y}}(B)\right\rangle= & \sqrt{\frac{1}{2 L}}\left[\sum_{k}^{\prime} \alpha_{k, \downarrow} \hat{a}_{k, \downarrow}^{+} \hat{d}_{\uparrow}^{+}|\mathrm{FS}\rangle\left|\operatorname{vac}_{d}\right\rangle\right. \\
& \left.-\sum_{k}^{\prime \prime} \alpha_{k, \uparrow} \hat{a}_{k, \uparrow}^{+} \hat{d}_{\downarrow}^{+}|\mathrm{FS}\rangle\left|\operatorname{vac}_{d}\right\rangle\right] .
\end{aligned}
$$

Since the Fermi sea depends on the magnetic field, the prime on the sum restricts the $k$ values to $\epsilon_{k}>-\epsilon_{\mathrm{F}}$, the double prime indicates $\epsilon_{k}>\epsilon_{\mathrm{F}}>0$, where $\epsilon_{\mathrm{F}}$ is a function of the magnetic energy scale $B>0$.

\section{B. Variational ground-state energy}

\section{Energy equation}

The calculations are carried out in Appendix A4. We abbreviate the principal-value integral

$$
F_{1}(x, B)=f_{B}^{1} d \omega \rho_{0}(\omega) \frac{1}{\omega-x},
$$

whereby we assume throughout that $0 \leqslant B<1$, i.e., the host electrons are not fully polarized. Note that Eq. (A41) permits to set $\epsilon_{\mathrm{F}}=B$ in our further considerations.

The Yosida ground-state energy $\lambda=e_{0}^{\mathrm{Y}}\left(J_{\mathrm{K}}, B\right)$ follows from the solution of the implicit equation

$$
\left(1-\frac{J_{\mathrm{K}} F_{+}}{4}\right)\left(1-\frac{J_{\mathrm{K}} F_{-}}{4}\right)-\frac{J_{\mathrm{K}}^{2} F_{+} F_{-}}{4}=0,
$$

where we abbreviated $F_{+} \equiv F_{1}\left(\lambda+J_{\mathrm{K}} s_{0} / 2, B\right)$ and $F_{-} \equiv$ $F_{1}\left(\lambda-J_{\mathrm{K}} s_{0} / 2,-B\right)$. In one spatial dimension we have 
$s_{0}(B)=(1 / \pi) \arcsin (B)$ from Eq. (A34) and

$$
F_{1}(x, B)=\frac{1}{\pi \sqrt{1-x^{2}}} \ln \left[\frac{1-B x+\sqrt{\left(1-B^{2}\right)\left(1-x^{2}\right)}}{B-x}\right] .
$$

Equation (56) provides a solution only for $B \leqslant B_{\mathrm{c}}^{\mathrm{Y}}\left(J_{\mathrm{K}}\right)$ above which the Yosida state becomes unstable. This problem does not occur in the Gutzwiller description so that we do not extend the Yosida state to the region $B>B_{\mathrm{c}}^{\mathrm{Y}}\left(J_{\mathrm{K}}\right)$.

\section{Ground-state energy at zero field}

At $B=0$, Eq. (56) simplifies to

$$
\begin{aligned}
& F(\lambda)=\frac{4}{3 J_{\mathrm{K}}}, \\
& F(\lambda) \equiv F_{1}(\lambda, 0)=\frac{1}{\pi \sqrt{1-\lambda^{2}}} \ln \left[\frac{1+\sqrt{1-\lambda^{2}}}{-\lambda}\right]
\end{aligned}
$$

for the ground-state energy $\lambda=e_{0}^{\mathrm{Y}}\left(J_{\mathrm{K}}\right)<0$. In general, the solution of Eq. (58) must be determined numerically.

a. Small Kondo couplings. For small $|E|$, we can address a general density of states because

$$
\begin{aligned}
F(\lambda) & =\rho_{0}(0) \int_{0}^{1} d \omega \frac{1}{\omega-\lambda}+\int_{0}^{1} d \omega \frac{\rho_{0}(\omega)-\rho_{0}(0)}{\omega-\lambda} \\
& \approx \rho_{0}(0)[-\ln (-\lambda)+\ln (C)]+\mathcal{O}(\lambda)
\end{aligned}
$$

for $|\lambda| \ll 1$. Here we introduced the regularized first negative moment of the density of states

$$
\ln (C)=-\int_{-1}^{0} \frac{d \omega}{\omega}\left(\frac{\rho_{0}(\omega)}{\rho_{0}(0)}-1\right)
$$

For a constant density of states we have $C^{\text {const }}=1$ by definition. For the one-dimensional density of states (8) we find $C^{d=1}=2$.

To leading order we must solve

$$
-\rho_{0}(0) \ln (|E| / C)=\frac{4}{3 J_{\mathrm{K}}}
$$

so that

$$
e_{0}^{\mathrm{Y}}\left(J_{\mathrm{K}} \ll 1\right)=-C \exp \left(-\frac{4}{3 \rho_{0}(0) J_{\mathrm{K}}}\right)
$$

results from the Yosida wave function for small Kondo couplings. The structure of the density of states only enters via the prefactor $C$. A comparison with the exact second-order expression (34) shows that the exponentially small variational bound provided by the Yosida wave function is rather poor.

b. Large Kondo couplings. For large Kondo couplings, the structure of the density of states matters and we restrict ourselves to the one-dimensional case. For large $|E|$ we must solve to leading and next-to-leading order

$$
\frac{1}{2|E|}-\frac{1}{\pi E^{2}}=\frac{4}{3 J_{\mathrm{K}}}
$$

So that

$$
e_{0}^{\mathrm{Y}}\left(J_{\mathrm{K}} \gg 1\right)=-\frac{3 J_{\mathrm{K}}}{8}+\frac{2}{\pi}
$$

results from the Yosida wave function for large Kondo couplings. The comparison with the perturbative strong-coupling result (42) shows that the Yosida wave function does not become exact for $J_{K} \gg 1$. This indicates that the Yosida state does not properly describe the strong-coupling singlet state.

\section{Zero-field susceptibilities}

The calculations are carried out in Appendix A 5. Here, we summarize the results for the various zero-field susceptibilities.

\section{Zero-field impurity spin susceptibility}

To obtain the zero-field impurity spin susceptibilities, we can replace $e_{0}^{\mathrm{Y}}\left(J_{\mathrm{K}}, B\right)$ and $e_{0, \text { loc }}^{\mathrm{Y}}\left(J_{\mathrm{K}}, B\right)$ by $e_{0}^{\mathrm{Y}}\left(J_{\mathrm{K}}\right)$ in Eqs. (A51) and (A52); corrections are of the order $B^{2}$ because the impurity spin polarization vanishes at $B=0$. Using Mathematica [29] and Eq. (58) in Eq. (15) we find

$$
\frac{\chi_{0}^{S, \mathrm{Y}}\left(J_{\mathrm{K}}\right)}{\left(g_{e} \mu_{\mathrm{B}}\right)^{2}}=\left(3 J_{\mathrm{K}}\right) \frac{2 \pi\left(3-\left[e_{0}^{\mathrm{Y}}\left(J_{\mathrm{K}}\right)\right]^{2}\right)-3 J_{\mathrm{K}}}{128 \pi^{2}\left(1-\left[e_{0}^{\mathrm{Y}}\left(J_{\mathrm{K}}\right)\right]^{2}\right)\left|e_{0}^{\mathrm{Y}}\left(J_{\mathrm{K}}\right)\right|}
$$

for the zero-field impurity spin susceptibility in the presence of a global field, and

$$
\frac{\chi_{0, \text { loc }}^{S, \mathrm{Y}}\left(J_{\mathrm{K}}\right)}{\left(g_{e} \mu_{\mathrm{B}}\right)^{2}}=\frac{3\left(3 J_{\mathrm{K}}-4 \pi\left[e_{0}^{\mathrm{Y}}\left(J_{\mathrm{K}}\right)\right]^{2}\right)}{64 \pi\left(1-\left[e_{0}^{\mathrm{Y}}\left(J_{\mathrm{K}}\right)\right]^{2}\right)\left|e_{0}^{\mathrm{Y}}\left(J_{\mathrm{K}}\right)\right|}
$$

for the zero-field impurity spin susceptibility in the presence of a local field for the one-dimensional density of states (8).

a. Small Kondo couplings. The Yosida energy is exponentially small, $e_{0}^{\mathrm{Y}}\left(J_{\mathrm{K}}\right) \approx-C \exp \left[-4 /\left(3 \rho_{0}(0) J_{\mathrm{K}}\right)\right]$, so that we obtain

$$
\begin{aligned}
& \frac{\chi_{0}^{S, \mathrm{Y}}\left(J_{\mathrm{K}} \ll 1\right)}{\left(g_{e} \mu_{\mathrm{B}}\right)^{2}} \approx \frac{\chi_{0, \mathrm{loc}}^{S, \mathrm{Y}}\left(J_{\mathrm{K}} \ll 1\right)}{\left(g_{e} \mu_{\mathrm{B}}\right)^{2}}\left(1-\frac{\rho_{0}(0) J_{\mathrm{K}}}{2}\right), \\
& \frac{\chi_{0, \mathrm{loc}}^{S, \mathrm{Y}}\left(J_{\mathrm{K}} \ll 1\right)}{\left(g_{e} \mu_{\mathrm{B}}\right)^{2}} \approx \frac{9 \rho_{0}(0) J_{\mathrm{K}} e^{4 /\left(3 \rho_{0}(0) J_{\mathrm{K}}\right)}}{64 C},
\end{aligned}
$$

which are identical up to a correction factor that goes to unity for $\rho_{0}(0) J_{K} \rightarrow 0$. The zero-field impurity spin susceptibilities display an exponential increase for small Kondo couplings, as is characteristic for the Kondo model.

b. Large Kondo couplings. For large Kondo couplings $J_{\mathrm{K}} \gg 1$, we have $e_{0}^{\mathrm{Y}}\left(J_{\mathrm{K}}\right) \approx-3 J_{\mathrm{K}} / 8+2 / \pi$ in one dimension so that we find

$$
\begin{gathered}
\frac{\chi_{0}^{S, \mathrm{Y}}\left(J_{\mathrm{K}} \gg 1\right)}{\left(g_{e} \mu_{\mathrm{B}}\right)^{2}} \approx \frac{1}{8 \pi}+\frac{2}{\pi^{2} J_{\mathrm{K}}}, \\
\frac{\chi_{0, \mathrm{loc}}^{S, \mathrm{Y}}\left(J_{\mathrm{K}} \gg 1\right)}{\left(g_{e} \mu_{\mathrm{B}}\right)^{2}} \approx \frac{1}{2 J_{\mathrm{K}}} .
\end{gathered}
$$

Since the Yosida state does not become exact for large Kondo couplings, the zero-field impurity spin susceptibility for a global field does not vanish for $J_{\mathrm{K}} \rightarrow \infty$. The corresponding susceptibility for a local field behaves properly, and even reproduces the exact result, as derived in Sec. VI.

\section{Zero-field impurity-induced susceptibility}

Using Mathematica [29] we find the impurity-induced magnetic susceptibility at zero field from Eq. (A58) 


$$
\begin{aligned}
& {\left[\lambda \equiv e_{0}^{\mathrm{Y}}\left(J_{\mathrm{K}}\right)\right]} \\
& \begin{aligned}
\frac{\chi_{0}^{\mathrm{ii}, \mathrm{Y}}\left(J_{\mathrm{K}}\right)}{\left(g_{e} \mu_{\mathrm{B}}\right)^{2}}= & \frac{J_{\mathrm{K}} C\left(\lambda, J_{\mathrm{K}}\right)}{128 \lambda\left(\lambda^{2}-1\right) \pi^{3}\left(4 \lambda^{2} \pi-3 J_{\mathrm{K}}\right)}, \\
C\left(\lambda, J_{\mathrm{K}}\right)= & 9 J_{\mathrm{K}}^{3}+12 J_{\mathrm{K}}^{2}\left(9 \lambda^{2}-5\right) \pi \\
& +4 J_{\mathrm{K}}\left(33-62 \lambda^{2}+9 \lambda^{4}\right) \pi^{2}-96\left(\lambda^{2}-1\right)^{2} \pi^{3},
\end{aligned}
\end{aligned}
$$

where we used Eq. (58) to simplify the expressions. In the presence of a local field, Eq. (A61) leads to

$$
\frac{\chi_{0, \mathrm{loc}}^{\mathrm{ii}, \mathrm{l}}\left(J_{\mathrm{K}}\right)}{\left(g_{e} \mu_{\mathrm{B}}\right)^{2}}=\frac{9 J_{\mathrm{K}}^{2}+24 \pi J_{\mathrm{K}}\left(3 \lambda^{2}-1\right)-16 \pi^{2} \lambda^{2}\left(2+3 \lambda^{2}\right)}{32 \lambda\left(\lambda^{2}-1\right) \pi\left(-3 J_{\mathrm{K}}+4 \pi \lambda^{2}\right)} .
$$

a. Small Kondo couplings. The Yosida energy is exponentially small $e_{0}^{\mathrm{Y}}\left(J_{\mathrm{K}}\right) \approx-C \exp \left[-4 \pi /\left(3 J_{\mathrm{K}}\right)\right]$, so that

$$
\begin{aligned}
\frac{\chi_{0}^{\mathrm{ii}, \mathrm{Y}}\left(J_{\mathrm{K}} \ll 1\right)}{\left(g_{e} \mu_{\mathrm{B}}\right)^{2}} \approx & \frac{e^{4 /\left(3 \rho_{0}(0) J_{\mathrm{K}}\right)}}{4 C} \\
& \times\left(1-\frac{11}{8} \rho_{0}(0) J_{\mathrm{K}}+\frac{5}{8}\left(\rho_{0}(0) J_{\mathrm{K}}\right)^{2}\right)
\end{aligned}
$$

with corrections of order $J_{\mathrm{K}}^{3}$, and

$$
\frac{\chi_{0, \mathrm{loc}}^{\mathrm{ii}, \mathrm{Y}}\left(J_{\mathrm{K}} \ll 1\right)}{\left(g_{e} \mu_{\mathrm{B}}\right)^{2}} \approx \frac{e^{4 /\left(3 \rho_{0}(0) J_{\mathrm{K}}\right)}}{4 C}\left(1-\frac{3}{8} \rho_{0}(0) J_{\mathrm{K}}\right)
$$

with exponentially small corrections. Thus, the zero-field impurity-induced susceptibility has the same exponential prefactor as in the case of a global field but the correction factor is different already in linear order.

The zero-field susceptibility is exponentially large for small $J_{\mathrm{K}}$, in qualitative agreement with the exact solution. However, the exponent is not quite correct, namely, the factor $\frac{4}{3}$ should be replaced by unity. Moreover, the exact susceptibility contains a correction factor proportional to $\sqrt{J_{\mathrm{K}} / \pi}$ (see Sec. VII).

Note that the shape of the density of states only enters through the prefactor $C$ that appears in the Yosida ground-state energy. Thus, the algebraic correction terms in Eqs. (72) and (73) are universal in the sense that they do not depend on the shape of the host-electron density of states. This behavior is also seen in the exact zero-field susceptibilities (see Sec. IX).

b. Large Kondo couplings. For large Kondo couplings $J_{\mathrm{K}} \gg 1$, we have $e_{0}^{\mathrm{Y}}\left(J_{\mathrm{K}}\right) \approx-3 J_{\mathrm{K}} / 8+2 / \pi$ in one dimension so that

$$
\frac{\chi_{0}^{\mathrm{ii}, \mathrm{Y}}\left(J_{\mathrm{K}} \gg 1\right)}{\left(g_{e} \mu_{\mathrm{B}}\right)^{2}} \approx-\frac{3 J_{\mathrm{K}}}{16 \pi^{2}}+\frac{\pi^{2}-12}{2 \pi^{3}}+\mathcal{O}\left(1 / J_{\mathrm{K}}\right)<0 .
$$

Since the Yosida wave function does not describe the local spin-singlet state properly, the susceptibility becomes negative for large Kondo couplings which indicates that the Yosida state is unstable for $J_{\mathrm{K}}>J_{\mathrm{K}, \mathrm{c}}^{\mathrm{Y}} \approx 3.7543$. The instability point is obtained from a numerical solution of $\chi_{0}^{\mathrm{ii}, \mathrm{Y}}\left(J_{\mathrm{K}, \mathrm{c}}^{\mathrm{Y}}\right)=0$. At $J_{\mathrm{K}}=J_{\mathrm{K}, \mathrm{c}}^{\mathrm{Y}}$, the critical external field vanishes, $B_{\mathrm{c}}^{\mathrm{Y}}\left(J_{\mathrm{K}, \mathrm{c}}^{\mathrm{Y}}\right)=0$.

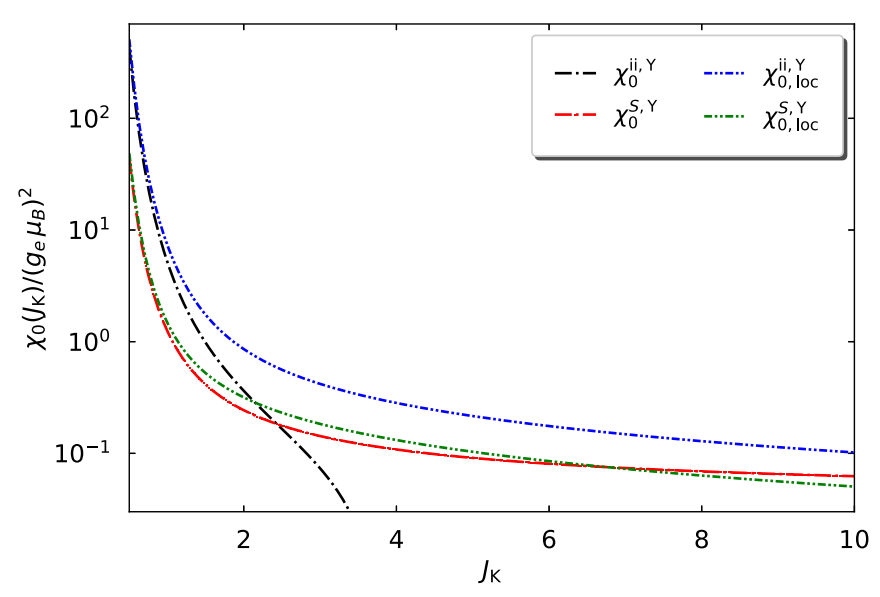

FIG. 1. Zero-field impurity spin susceptibility $\chi_{0}^{S, \mathrm{Y}} /\left(g_{e} \mu_{\mathrm{B}}\right)^{2}$ [Eqs. (65) and (66)] and zero-field impurity-induced susceptibility $\chi_{0}^{\mathrm{ii}, \mathrm{Y}} /\left(g_{e} \mu_{\mathrm{B}}\right)^{2}$ [Eqs. (70) and (71)] for global/local magnetic fields as a function of $J_{\mathrm{K}}$ of the one-dimensional symmetric Kondo model from the Yosida wave function. The zero-field impurity-induced susceptibility becomes negative at $J_{\mathrm{K}}=J_{\mathrm{K}, \mathrm{c}}^{\mathrm{Y}} \approx 3.7543$.

For large Kondo couplings $J_{\mathrm{K}} \gg 1$, we have $e_{0}^{\mathrm{Y}}\left(J_{\mathrm{K}}\right) \approx$ $-3 J_{\mathrm{K}} / 8+2 / \pi$ so that

$$
\frac{\chi_{0, \text { loc }}^{\mathrm{ii}, \mathrm{Y}}\left(J_{\mathrm{K}} \gg 1\right)}{\left(g_{e} \mu_{\mathrm{B}}\right)^{2}} \approx \frac{1}{J_{\mathrm{K}}}+\mathcal{O}\left(J_{\mathrm{K}}^{-3}\right) .
$$

This result is qualitatively correct. Note, however, that the Yosida wave function fails to reproduce the exact equivalence of the zero-field impurity-induced susceptibility for a local field and the zero-field impurity spin susceptibility [Eq. (24)].

In Fig. 1 we show the corresponding zero-field susceptibilities. They all display an exponential increase for small $J_{\mathrm{K}}$, and only differ in the preexponential factor. For the impurity spin susceptibility in the Yosida wave function, this factor is proportional to $J_{\mathrm{K}}$ and also numerically small [see Eq. (67)]. Therefore, the impurity spin susceptibility is substantially smaller than the impurity-induced susceptibility; this is an artifact of the Yosida wave function.

For large Kondo couplings $J_{\mathrm{K}}>1$, the impurity-induced susceptibility becomes negative for $J_{\mathrm{K}}>J_{\mathrm{K}, \mathrm{c}}^{\mathrm{Y}}$, i.e., the Yosida state becomes unstable against a state with a locally broken symmetry. The other susceptibilities remain positive for all $J_{\mathrm{K}}$. The impurity spin susceptibility becomes constant for large $J_{\mathrm{K}}$ [see Eq. (68)] which is at odds with the exact solution. The local susceptibilities are qualitatively correct for large couplings inasmuch they decay to zero for strong couplings [see Eqs. (69) and (75)]. In fact, the impurity spin susceptibility from the Yosida wave function in the presence of a local field $\chi_{0, \mathrm{Yoc}}^{S, \mathrm{Y}}\left(J_{\mathrm{K}}\right)$ [Eq. (69)] becomes exact in the limit of strong coupling (see Sec. IX).

\section{GUTZWILLER WAVE FUNCTION}

As the third and last analytic variational approach, we study the Gutzwiller wave function. It becomes exact in the limit of large Kondo couplings and provides a very good variational upper bound for the ground-state energy for all Kondo couplings. However, for weak couplings it describes 
a symmetry-broken state with an oriented moment on the impurity, and a transition to the paramagnetic state at $J_{\mathrm{K}, \mathrm{c}}^{\mathrm{G}}$ that is not contained in the exact solution of the model.

\section{A. Gutzwiller variational state}

We define the Gutzwiller variational state $[18,19]$

$$
\left|\Psi_{\mathrm{G}}\right\rangle=\hat{P}_{\mathrm{G}}|\Phi\rangle,
$$

where $|\Phi\rangle$ is a normalized single-particle product state to be determined variationally. At half band filling we have

$$
n_{\sigma}^{d, 0}=\left\langle\Phi\left|\hat{n}_{\sigma}^{d}\right| \Phi\right\rangle=\frac{1}{2}+\sigma_{n} m,
$$

where $\sigma_{n}=1$ for $\sigma=\uparrow$ and $\sigma_{n}=-1$ for $\sigma=\downarrow$, and $0 \leqslant$ $m<\frac{1}{2}$ is the impurity spin polarization in the single-particle product state $|\Phi\rangle$ :

$$
m=\frac{1}{2}\left\langle\Phi\left|\hat{n}_{\uparrow}^{d}-\hat{n}_{\downarrow}^{d}\right| \Phi\right\rangle .
$$

For a complete Gutzwiller projection, we choose

$$
\hat{P}_{\mathrm{G}}=\lambda_{\uparrow} \hat{m}_{\uparrow}^{d}+\lambda_{\downarrow} \hat{m}_{\downarrow}^{d}, \quad \hat{m}_{\sigma}^{d}=\hat{n}_{\sigma}^{d}\left(1-\hat{n}_{\bar{\sigma}}^{d}\right),
$$

where we use $\bar{\uparrow}=\downarrow$ and $\bar{\downarrow}=\uparrow$. Moreover, we demand

$$
\hat{P}_{\mathrm{G}}^{2}=1+x\left(\hat{n}_{\uparrow}^{d}-n_{\uparrow}^{d, 0}\right)\left(\hat{n}_{\downarrow}^{d}-n_{\downarrow}^{d, 0}\right)
$$

which does not restrict the variational freedom but simplifies the evaluation of the wave function; for a thorough discussion, see Ref. [30]. Thus, we have to solve

$$
\begin{aligned}
\lambda_{\uparrow}^{2} \hat{m}_{\uparrow}^{d}+\lambda_{\downarrow}^{2} \hat{m}_{\downarrow}^{d} & =\lambda_{\uparrow}^{2} \hat{n}_{\uparrow}^{d}+\lambda_{\downarrow}^{2} \hat{n}_{\downarrow}^{d}-\left(\lambda_{\uparrow}^{2}+\lambda_{\downarrow}^{2}\right) \hat{n}_{\uparrow}^{d} \hat{n}_{\downarrow}^{d} \\
& \stackrel{!}{=} 1+x\left(\hat{n}_{\uparrow}^{d}-n_{\uparrow}^{d, 0}\right)\left(\hat{n}_{\downarrow}^{d}-n_{\downarrow}^{d, 0}\right) .
\end{aligned}
$$

The solution reads as

$$
\begin{aligned}
x & =-\frac{1}{n_{\uparrow}^{d, 0} n_{\downarrow}^{d, 0}}=-\frac{4}{1-4 m^{2}}, \\
\lambda_{\sigma}^{2} & =\frac{1}{n_{\sigma}^{d, 0}}=\frac{2}{1+2 \sigma_{n} m} .
\end{aligned}
$$

Before we proceed, we note the useful relations

$$
\begin{aligned}
\hat{P}_{\mathrm{G}} \hat{d}_{\sigma}^{+} \hat{d}_{\bar{\sigma}} \hat{P}_{\mathrm{G}} & =\lambda_{\sigma} \lambda_{\bar{\sigma}} \hat{d}_{\sigma}^{+} \hat{d}_{\bar{\sigma}}, \\
\hat{P}_{\mathrm{G}}\left(\hat{n}_{\uparrow}^{d}-\hat{n}_{\downarrow}^{d}\right) \hat{P}_{\mathrm{G}} & =\lambda_{\uparrow}^{2} \hat{m}_{\uparrow}^{d}-\lambda_{\downarrow}^{2} \hat{m}_{\downarrow}^{d} \\
& =\frac{\hat{n}_{\uparrow}^{d}}{n_{\uparrow}^{d, 0}}-\frac{\hat{n}_{\downarrow}^{d}}{n_{\downarrow}^{d, 0}}+2 m \frac{\hat{n}_{\uparrow}^{d} \hat{n}_{\downarrow}^{d}}{n_{\uparrow}^{d, 0} n_{\downarrow}^{d, 0}} .
\end{aligned}
$$

\section{B. Ground-state energy}

The calculation of expectation values and the variational optimization of the energy functional is presented in Appendices A 6 and A 7. It requires the solution of an effective noninteracting single-impurity Anderson model that is characterized by a local hybridization parameter $V$.

\section{Paramagnetic Gutzwiller state}

For $m=0$, the ground-state energy for the non-interacting single-impurity Anderson model is known explicitly for all relevant cases. For example, in one dimension it reads as [28]

$$
\begin{aligned}
e_{0}^{\mathrm{A}}(V)= & \frac{1}{\pi}\left[-\pi+2 v_{+} \arctan \left(\frac{1}{v_{-}}\right)+v_{-} \ln \left(\frac{v_{+}-1}{v_{+}+1}\right)\right] \\
& +2\left(1-v_{+}\right), \\
v_{ \pm}= & \sqrt{\frac{\sqrt{1+4 V^{4}} \pm 1}{2}} .
\end{aligned}
$$

The Hellmann-Feynman theorem then gives

$$
\frac{\partial e_{0}^{\mathrm{A}}(V)}{\partial V}=-\frac{8 V}{3 J_{\mathrm{K}}} .
$$

The self-consistency equation (86) defines $V\left(J_{\mathrm{K}}\right)$ as a function of $J_{\mathrm{K}}$.

The Gutzwiller variational energy for the Kondo model becomes

$$
e_{0}^{\mathrm{G}}\left(J_{\mathrm{K}}\right)=e_{0}^{\mathrm{A}}\left(V\left(J_{\mathrm{K}}\right)\right)+\frac{4\left[V\left(J_{\mathrm{K}}\right)\right]^{2}}{3 J_{\mathrm{K}}} .
$$

In general, the Gutzwiller variational energy for the Kondo model must be determined numerically.

a. Small Kondo couplings. For $J_{\mathrm{K}} \ll 1$ and thus $V \ll 1$, we can approximate

$$
e_{0}^{\mathrm{A}}(V \ll 1) \approx \frac{2 V^{2}}{\pi}\left[\ln \left(V^{2} / 2\right)-1\right]
$$

in one dimension so that the self-consistency equation (86) becomes

$$
-\frac{2 \pi}{3 J_{\mathrm{K}}}=\ln \left(V^{2} / 2\right), \quad V^{2}=2 \exp \left(-\frac{2 \pi}{3 J_{\mathrm{K}}}\right) .
$$

Therefore, the Gutzwiller estimate for the ground-state energy at small Kondo couplings becomes

$$
e_{0}^{\mathrm{G}}\left(J_{\mathrm{K}} \ll 1\right)=-\frac{4}{\pi} \exp \left(-\frac{2}{3 \rho_{0}(0) J_{\mathrm{K}}}\right) .
$$

This is much smaller than the Yosida energy equation (62), and even smaller than the value for the Yosida-Yoshimori wave function $[17,31]$

$$
e_{0}^{\mathrm{Y}, \mathrm{Y}}\left(J_{\mathrm{K}} \ll 1\right) \sim-\exp \left(-\frac{1}{\rho_{0}(0) J_{\mathrm{K}}}\right) .
$$

This is not surprising because both variational states miss the actually quadratic dependence of the ground-state energy on $J_{\mathrm{K}}$ for small interaction strengths $e_{0}\left(J_{\mathrm{K}}\right) \sim-J_{\mathrm{K}}^{2}$ [see Eq. (34)].

b. Large Kondo couplings. For $J_{\mathrm{K}} \gg 1$ we can approximate

$e_{0}^{\mathrm{A}}(V \gg 1) \approx-2 V+1-\frac{1}{2 V}+\frac{2}{3 \pi V^{2}}-\frac{1}{16 V^{3}}+\mathcal{O}\left(1 / V^{5}\right)$

in one dimension. The self-consistency equation (86) becomes

$$
\begin{aligned}
-\frac{4 V}{3 J_{\mathrm{K}}} & =-1+\frac{1}{4 V^{2}}-\frac{2}{3 \pi V^{3}}+\frac{3}{32 V^{4}}+\mathcal{O}\left(1 / V^{6}\right), \\
V & =\frac{3 J_{\mathrm{K}}}{4}-\frac{1}{3 J_{\mathrm{K}}}+\frac{32}{27 \pi J_{\mathrm{K}}^{2}}-\frac{14}{27 J_{\mathrm{K}}^{3}}+\mathcal{O}\left(1 / J_{\mathrm{K}}^{5}\right) .
\end{aligned}
$$


Therefore, the Gutzwiller estimate for the ground-state energy at large Kondo couplings reads as

$$
e_{0}^{\mathrm{G}}\left(J_{\mathrm{K}} \gg 1\right)=-\frac{3 J_{\mathrm{K}}}{4}+1-\frac{2}{3 J_{\mathrm{K}}}+\frac{32}{27 \pi J_{\mathrm{K}}^{2}}-\frac{8}{27 J_{\mathrm{K}}^{3}},
$$

up to and including third order in $1 / J_{\mathrm{K}}$. This is much smaller than the Yosida energy (64) and is actually exact, up to corrections of the order $1 / J_{\mathrm{K}}$ [see Eq. (42)]. Below, we argue that the first- and second-order corrections in $1 / J_{\mathrm{K}}$ are also exact.

The local spin correlation is obtained from the variational Hellmann-Feynman theorem. For large $J_{\mathrm{K}}$ we find

$$
C_{0}^{S, \mathrm{G}}\left(J_{\mathrm{K}} \gg 1\right)=-\frac{3}{4}+\frac{2}{3 J_{\mathrm{K}}^{2}}-\frac{64}{27 \pi J_{\mathrm{K}}^{3}}+\mathcal{O}\left(1 / J_{\mathrm{K}}^{4}\right) .
$$

\section{Magnetic Gutzwiller state for weak coupling}

As shown in Appendix A 7, the numerical optimization of the variational parameters leads to

$$
e_{0}^{\mathrm{G}}\left(J_{\mathrm{K}}\right) \approx-0.0905 J_{\mathrm{K}}^{2}-0.051 J_{\mathrm{K}}^{3}-0.05 J_{\mathrm{K}}^{4}
$$

for the Gutzwiller variational energy for $J_{\mathrm{K}} \lesssim 0.4$.

The quadratic coefficient from the magnetic Gutzwiller wave function can be compared with the exact result from perturbation theory $e_{0}\left(J_{\mathrm{K}}\right) \approx-3 J_{\mathrm{K}}^{2} / 32=-0.09375 J_{\mathrm{K}}^{2}$ [see Eq. (34)]. The magnetic Gutzwiller states account for $96.5 \%$ of the correlation energy. Hence, the magnetic Gutzwiller provides an excellent energy estimate but fails to describe the physics properly because it breaks the local symmetry $m>0$ for $B=0^{+}$.

\section{Zero-field susceptibilities}

The calculations are carried out in Appendix A 8. Here, we summarize the results for the various zero-field susceptibilities in the strong-coupling limit.

\section{Five equations}

The calculation of the zero-field susceptibilities from the Gutzwiller wave function requires the solution of a $5 \times 5$ matrix problem

$$
\underline{\underline{M}} \cdot \underline{v}=\underline{g},
$$

where $g_{\text {loc }}^{\mathrm{T}}=(1,0,0,0,0)$ for a local field, and $\underline{g}^{\mathrm{T}}=$ $\left(1,0, g_{3}, g_{4}, g_{5}\right)$ for a global field whose nontrivial entries $g_{3}, g_{4}, g_{5}$ are known functions of $V$, and $V\left(J_{\mathrm{K}}\right)$ follows from Eq. (86) (see Appendix A 8). Likewise, the entries of the $5 \times 5$ matrix $\underline{\underline{M}}$ are known functions of $V$ and $J_{\mathrm{K}}$. The vector

$$
\underline{v}=\left(\begin{array}{c}
\bar{\omega}_{p} \\
\bar{E}_{d} \\
\bar{K} \\
\bar{M}_{0} \\
\chi
\end{array}\right)
$$

contains the five unknowns that determine the susceptibilities

$$
\begin{gathered}
\frac{\chi_{0}^{S, \mathrm{G}}\left(J_{\mathrm{K}}, B\right)}{\left(g_{e} \mu_{\mathrm{B}}\right)^{2}}=\chi, \\
\frac{\chi_{0}^{\mathrm{ii}, \mathrm{G}}\left(J_{\mathrm{K}}\right)}{\left(g_{e} \mu_{\mathrm{B}}\right)^{2}}=\frac{\bar{E}_{d}}{2 \pi V^{2}} .
\end{gathered}
$$

The choice of $\underline{g}$ determines whether the external field is applied globally or locally.

Although Mathematica [29] provides an analytic solution of the linear problem, the expressions are very lengthy and not illuminating. Eventually, we evaluate them numerically.

\section{Strong-coupling limit}

As shown in Appendix A 8, compact results can be obtained for $J_{\mathrm{K}} \gg 1$. For the zero-field impurity spin susceptibility we find

$$
\begin{gathered}
\frac{\chi_{0}^{S, \mathrm{G}}\left(J_{\mathrm{K}} \gg 1\right)}{\left(g_{e} \mu_{\mathrm{B}}\right)^{2}}=\frac{20}{9 \pi J_{\mathrm{K}}^{2}}+\mathcal{O}\left(1 / J_{\mathrm{K}}^{4}\right), \\
\frac{\chi_{0, \text { loc }}^{S, \mathrm{G}}\left(J_{\mathrm{K}} \gg 1\right)}{\left(g_{e} \mu_{\mathrm{B}}\right)^{2}}=\frac{1}{2 J_{\mathrm{K}}}+\frac{28}{27 J_{\mathrm{K}}^{3}}+\mathcal{O}\left(1 / J_{\mathrm{K}}^{4}\right)
\end{gathered}
$$

in the presence of a global and a local field, respectively.

For the zero-field impurity-induced susceptibilities, the Gutzwiller result for strong coupling reads as

$$
\begin{gathered}
\frac{\chi_{0}^{\mathrm{ii}, \mathrm{G}}\left(J_{\mathrm{K}} \gg 1\right)}{\left(g_{e} \mu_{\mathrm{B}}\right)^{2}}=\frac{8}{9 \pi J_{\mathrm{K}}^{2}}+\frac{416}{81 \pi^{2} J_{\mathrm{K}}^{3}}+\mathcal{O}\left(1 / J_{\mathrm{K}}^{4}\right), \\
\frac{\chi_{0, \text { loc }}^{\mathrm{ii}, \mathrm{G}}\left(J_{\mathrm{K}} \gg 1\right)}{\left(g_{e} \mu_{\mathrm{B}}\right)^{2}}=\frac{20}{9 \pi J_{\mathrm{K}}^{2}}+\mathcal{O}\left(1 / J_{\mathrm{K}}^{4}\right)
\end{gathered}
$$

in the presence of a global and a local field, respectively.

Since the Gutzwiller wave function becomes the exact ground state for strong coupling, we argue that these results are correct to the indicated order. We shall confirm this assessment from the comparison with numerically exact data from NRG and DMRG in Sec. IX C.

\section{Critical interaction for the magnetic transition}

For $J_{\mathrm{K}}>J_{\mathrm{K}, \mathrm{c}}^{\mathrm{G}}$, the Gutzwiller state describes a spinisotropic state at the impurity site. For $J_{\mathrm{K}}<J_{\mathrm{K}, \mathrm{c}}^{\mathrm{G}}$, the local spin symmetry in the Gutzwiller state is spontaneously broken, i.e., $m>0$ is optimal even at $B=0^{+}$.

With the help of the zero-field spin susceptibility, the transition can accurately be identified because the determinant of the matrix $M\left(J_{\mathrm{K}}\right)$ changes sign at $J=J_{\mathrm{K}, \mathrm{c}}^{\mathrm{G}}$. Using Mathematica [29], the determinant as a function of $V$ can be calculated analytically but the expressions are lengthy. The solution of

$$
\operatorname{det}\left(\underline{\underline{M}}\left(V_{\mathrm{c}}\right)\right)=0
$$

is $V_{\mathrm{c}}=0.4559222509954975$ with $\operatorname{det}\left(\underline{\underline{M}}\left(V_{\mathrm{c}}\right)\right)=-3.8 \times$ $10^{-15}$, or

$$
J_{\mathrm{K}, \mathrm{c}}^{\mathrm{G}}=0.8392762432533198 .
$$

\section{Comparison of susceptibilities}

The paramagnetic Gutzwiller state is stable only for $J_{\mathrm{K}}>$ $J_{\mathrm{K}, \mathrm{c}}^{\mathrm{G}} \approx 0.839$ so that we focus on $J_{\mathrm{K}} \geqslant 1$. Since the Gutzwiller wave function becomes exact for $J_{\mathrm{K}} \rightarrow \infty$, all susceptibilities are positive and well behaved.

In Fig. 2 we show the global and local zero-field susceptibilities. As seen from the figure, the asymptotic formulas for the impurity spin susceptibilities, Eqs. (101), (102), (103), and (104), are applicable for $J_{\mathrm{K}} \gtrsim 4$. 


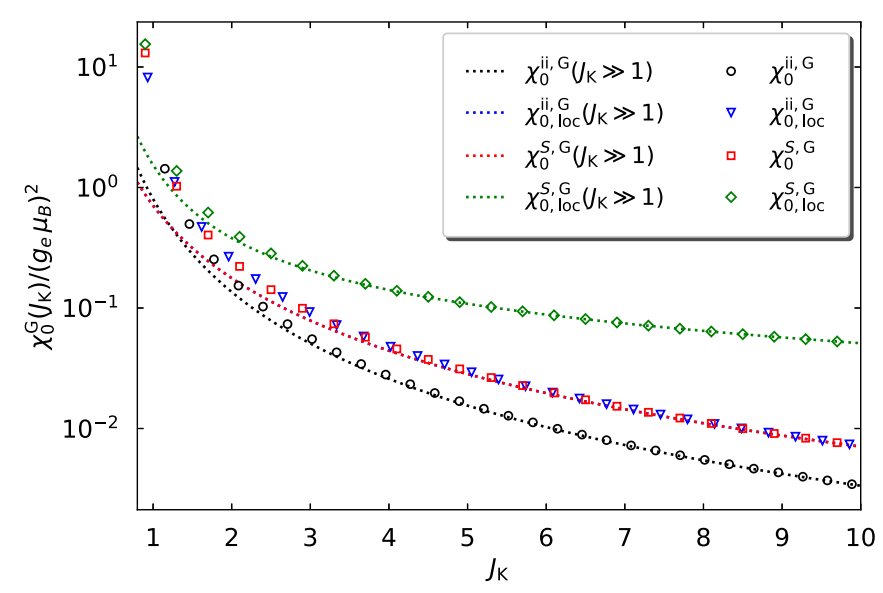

FIG. 2. Zero-field impurity spin susceptibility $\chi_{0}^{S, \mathrm{G}} /\left(g_{e} \mu_{\mathrm{B}}\right)^{2}$ [Eq. (99)] and zero-field impurity-induced susceptibility $\chi_{0}^{\mathrm{ii}, \mathrm{G}} /\left(g_{e} \mu_{\mathrm{B}}\right)^{2}$ [Eq. (100)] for global/local magnetic fields as a function of $J_{\mathrm{K}} \geqslant 1$ of the one-dimensional symmetric Kondo model from the Gutzwiller wave function. For comparison, we also include the limiting behavior for the susceptibilities, Eqs. (101), (102), (103), and (104).

Figure 2 also shows that the equivalence

$$
\chi_{0}^{S, \mathrm{G}}\left(J_{\mathrm{K}}\right)=\chi_{0, \mathrm{loc}}^{\mathrm{ii}, \mathrm{G}}\left(J_{\mathrm{K}}\right)
$$

holds for all $J_{\mathrm{K}}>1$. Therefore, the Gutzwiller approach respects the exact relation (24) at zero temperature. Indeed, as seen from the derivation in Appendix A 7, the Gutzwiller approach solves an effective noninteracting single-impurity Anderson model for which the exact relation (24) is readily shown to hold, too.

\section{BETHE-ANSATZ RESULTS}

Using Bethe ansatz, the Kondo model is solved for a linear dispersion relation with unit Fermi velocity in the wide-band limit, i.e., the dispersion relation $\epsilon^{\mathrm{BA}}(k)=k$ formally extends from $k_{-}=-\infty$ to $k_{+}=+\infty$. Therefore, an appropriate energy cutoff $D$ must be introduced in the Bethe-ansatz equations. This procedure is not unique. Therefore, there are two Bethe-ansatz solutions for the spin- $\frac{1}{2}$ Kondo model: first, the one discussed by Tsvelick and Wiegmann, referred to as TW [10], and, second, the one reviewed by Andrei, Furuya, and Lowenstein, referred to as AFL [11]. The basic Bethe-ansatz equations agree but the expressions for the parameters as a function of the Bethe-ansatz Kondo coupling $J_{\mathrm{K}}^{\mathrm{BA}} / D$ differ beyond leading order. For a lattice-regularized Bethe-ansatz solvable impurity model, see Ref. [32].

In this section we discuss the Bethe-ansatz results for the zero-field impurity-induced susceptibility and magnetization. As shown in Appendix B 5, the Bethe-ansatz solution leads to

$$
e_{0}^{\mathrm{BA}}\left(J_{\mathrm{K}}^{\mathrm{BA}}\right)=\mathcal{O}\left(\left(J_{\mathrm{K}}^{\mathrm{BA}}\right)^{3}\right)
$$

Thus, the Bethe-ansatz results cannot be used for comparison with the ground-state energy of the Kondo impurity on a chain.

\section{A. Zero-field impurity-induced magnetic susceptibility}

The Bethe ansatz leads to Eq. (4.30) of AFL [11] or Eq. (5.1.23) of TW [10] for the zero-field magnetic susceptibility

$$
\frac{\chi_{0}^{\mathrm{ii}}\left(J_{\mathrm{K}}\right)}{\left(g_{e} \mu_{\mathrm{B}}\right)^{2}}=\frac{1}{4 \pi T_{0}\left(J_{\mathrm{K}}^{\mathrm{BA}}\right)}
$$

in the limit of small $J_{\mathrm{K}}^{\mathrm{BA}}$ for a half-filled system.

The Bethe ansatz solves the Kondo model for a Kondo interaction strength $J_{\mathrm{K}}^{\mathrm{BA}}$ in the limit of an infinite bandwidth. To arrive at tangible results, a symmetric bandwidth cutoff, $|\epsilon|<D$, is imposed on the Bethe-ansatz equations, and periodic boundary conditions are implemented so that the electron density remains finite, $D_{\mathrm{AFL}} \equiv N^{e} / L=1 / 2$ at half band filling (see Appendix B 5). The corresponding bandwidth is $W^{\mathrm{BA}}=2 D$ with

$$
D \equiv K_{\mathrm{AFL}}=\pi D_{\mathrm{AFL}}=\frac{\pi}{2}
$$

so that the density of states is given by

$$
\rho_{0}=\frac{1}{2 D}=\frac{1}{\pi}
$$

at the Fermi energy $E_{\mathrm{F}}=0$, and for all $|\epsilon| \leqslant D$. Then, the low-temperature magnetic energy scale from Bethe ansatz is given by

$$
T_{0}\left(J_{\mathrm{K}}^{\mathrm{BA}}\right)=\frac{D}{\pi} \exp \left(-\frac{1}{\rho_{0} J_{\mathrm{K}}^{\mathrm{BA}}}\right) .
$$

The remaining problem is to express $T_{0}\left(J_{\mathrm{K}}^{\mathrm{BA}}\right)$ as a function of $J_{\mathrm{K}}$ or, equivalently, to find an explicit relation between $J_{\mathrm{K}}^{\mathrm{BA}}$ and $J_{\mathrm{K}}$. The existence of such a unique relation is thoroughly discussed in Sec. VI of AFL [11].

\section{B. Wilson's renormalization group}

Wilson's renormalization group [5] for the Kondo model starts from the lattice model in the thermodynamic limit with its energy cutoff parameter $\mathcal{D}=W / 2=1$ and Kondo coupling $J_{\mathrm{K}}$. By successively integrating out the high-energy degrees of freedom, the renormalization group flows to the Bethe-ansatz model with a linear dispersion relation around the Fermi energy and the coupling $J_{\mathrm{K}}^{\mathrm{BA}}$.

The renormalization group ( $R G$ ) transformation is actually performed on the Hamiltonian as well as on the matrix representation of the operators, both influencing the physical quantities such as the zero-field impurity-induced susceptibility. In his review, Eq. (IX.91) on p. 835 [5], Wilson provides the general series expansion for the zero-field impurity-induced susceptibility at zero temperature

$$
\begin{aligned}
\frac{\chi_{0}^{\mathrm{ii}}\left(J_{\mathrm{K}}\right)}{\left(g_{e} \mu_{\mathrm{B}}\right)^{2}} & =\frac{w}{4 \widetilde{D}(j)} \frac{\exp (1 / j)}{\sqrt{j}} \exp \left(-\alpha_{1} j-\sum_{n=2}^{\infty} \alpha_{n} j^{n}\right) \\
\frac{\widetilde{D}(j)}{\mathcal{D}} & =c_{0}+\sum_{n=1}^{\infty} c_{n} j^{n}, \quad j=\rho_{0}(0) J_{\mathrm{K}}
\end{aligned}
$$

where $\rho_{0}(0)$ is the density of states at the Fermi energy. In Ref. [5], $\alpha_{1}$ and $w$ were determined numerically for a constant density of states $w / 4 \approx 0.1032$ and $\alpha_{1}=1.5824$. 
AFL calculated the Wilson number $w$ analytically [11]

$$
w=\frac{w_{\mathrm{AFL}}}{\pi}=\frac{e^{\gamma_{\mathrm{E}}+1 / 4}}{\pi^{3 / 2}} \approx 0.410705
$$

with Euler's constant $\gamma_{\mathrm{E}} \approx 0.577216$. We concisely rederive $w$ in Appendix B 6.

The coefficients $c_{n}$ in Eq. (113) can be obtained from the high-temperature expansion of the zero-field impurityinduced susceptibility. To third order it reads as [see Eq. (IX.57) of Ref. [5]]

$$
\begin{aligned}
\frac{4 T \chi_{0}^{\mathrm{ii}}\left(T, J_{\mathrm{K}}\right)}{\left(g_{e} \mu_{\mathrm{B}}\right)^{2}} \approx & 1-j+j^{2} \ln [T / \widetilde{D}(j)] \\
& -j^{3}\left[(\ln [T / \widetilde{D}(j)])^{2}+\frac{1}{2} \ln [T / \widetilde{D}(j)] .\right.
\end{aligned}
$$

Indeed, to second order the comparison of Eq. (VI-78) of the Supplemental Material [23] with Eq. (115) gives

$$
\frac{\widetilde{D}(j)}{\mathcal{D}} \approx \frac{U C}{2} \equiv c_{0}
$$

with

$$
U=\frac{e^{3 / 4+\gamma_{\mathrm{E}}}}{\pi}=\sqrt{\pi e} w
$$

from Eq. (VI-58) and $C$ from Eq. (60). Thus, the prefactor of the susceptibility in Eq. (113) to leading order reads as

$$
\frac{w}{4 \widetilde{D}(j)} \approx \frac{w}{2 U C \mathcal{D}}=\frac{1}{2 \mathcal{D} C \sqrt{\pi e}} .
$$

This result does not contain the Wilson number but only the prefactor $1 /(2 \mathcal{D} C \sqrt{\pi e})$. This does not come as a surprise because we consider finite magnetic fields at zero temperature while $T_{\mathrm{K}}$ characterizes the zero-field susceptibility at finite temperatures. Note that the prefactor $c_{0}$ in Eq. (116) contains information about the host-electron density of states via the regularized first negative moment (60).

As shown in AFL [11], and rederived in Appendix B 6, the Kondo temperature $T_{\mathrm{K}}$ and the magnetic energy scale $T_{\mathrm{H}}=$ $\sqrt{\pi / e} T_{0}$ are related by

$$
T_{\mathrm{K}}=U T_{\mathrm{H}},
$$

with corrections of the order $\rho_{0}(0) J_{\mathrm{K}}$. Using Eqs. (109), (112), (113), and (118), we find

$$
T_{\mathrm{K}}=w \sqrt{\pi e} \frac{\mathcal{D} C}{2} \sqrt{\rho_{0}(0) J_{\mathrm{K}}} \exp \left(-\frac{1}{\rho_{0}(0) J_{\mathrm{K}}}\right),
$$

with corrections of the order $\rho_{0}(0) J_{\mathrm{K}}$. Thus, in Eq. (109)

$$
\frac{\chi_{0}^{\mathrm{ii}}\left(J_{\mathrm{K}}\right)}{\left(g_{e} \mu_{\mathrm{B}}\right)^{2}}=\frac{w}{4 T_{\mathrm{K}}},
$$

which is the familiar expression of the zero-temperature susceptibility in terms of the Kondo temperature $T_{\mathrm{K}}$ [see Eq. (4.58) of Hewson's book [4]].
In general, Eq. (113) can be cast into the form

$$
\begin{aligned}
& \frac{\chi_{0}^{\mathrm{ii}}\left(J_{\mathrm{K}}\right)}{\left(g_{e} \mu_{\mathrm{B}}\right)^{2}}=s_{0} \frac{\bar{\chi}_{0}(j)}{\left(g_{e} \mu_{\mathrm{B}}\right)^{2}}\left(1+\sum_{n=1}^{\infty} \frac{s_{n}}{s_{0}} j^{n}\right), \quad j=\rho_{0}(0) J_{\mathrm{K}} \\
& \frac{\bar{\chi}_{0}(j)}{\left(g_{e} \mu_{\mathrm{B}}\right)^{2}}=\frac{\exp (1 / j)}{\sqrt{j}}, \quad s_{0}=\frac{1}{2 \mathcal{D} C \sqrt{\pi e}} .
\end{aligned}
$$

To go beyond the leading order, i.e., to determine the coefficient $s_{1}$ in Eq. (122) analytically, requires the cumbersome calculation of the ground-state energy as a function of magnetic field $B$ to third order in $\rho_{0}(0) J_{\mathrm{K}}$. This is beyond the scope of our presentation.

The comparison of the zero-field impurity-induced susceptibility from the renormalization group in Eq. (122) with the corresponding Bethe-ansatz expressions (109) and (112) leads to the desired relation between $\left(D, J_{\mathrm{K}}^{\mathrm{BA}}\right)$ and $\left(\mathcal{D}, J_{\mathrm{K}}\right)$ :

$$
\begin{aligned}
\frac{1}{\rho_{0} J_{\mathrm{K}}^{\mathrm{BA}}}= & \frac{1}{\rho_{0}(0) J_{\mathrm{K}}}-\frac{1}{2} \ln \left(\rho_{0}(0) J_{\mathrm{K}}\right)+\ln \left(\sqrt{\frac{1}{\pi e}} \frac{2 D}{C \mathcal{D}}\right) \\
& +\ln \left[1+\sum_{n=1}^{\infty} \frac{s_{n}}{s_{0}}\left(\rho_{0}(0) J_{\mathrm{K}}\right)^{n}\right]
\end{aligned}
$$

with $\mathcal{D}=W / 2=1$ for the lattice model and $D=\pi / 2$ in the Bethe-ansatz solvable model.

In passing, we address the zero-field spin susceptibility of the single-impurity Anderson model (SIAM). For the SIAM, the Bethe ansatz gives [4]

$$
\frac{\chi_{0}^{\mathrm{ii}, \operatorname{SIAM}}(u)}{\left(g_{e} \mu_{\mathrm{B}}\right)^{2}}=\frac{1}{4 T_{L}(u)}\left[1+\int_{0}^{\pi /(2 u)} \frac{d x}{\sqrt{\pi x}} e^{x-\pi^{2} /(16 x)}\right]
$$

with

$$
T_{L}(u)=U_{\mathrm{H}} \sqrt{\frac{1}{2 u}} e^{-\pi u / 8+\pi /(2 u)}
$$

and $u=U_{\mathrm{H}} / \Gamma, \Gamma=4 V^{2} / W$ in terms of the Hubbard interaction $U_{\mathrm{H}}$ and the local hybridization $V$ in the SIAM with a constant density of states.

In the limit of large $u$, the integral in Eq. (124) becomes exponentially small so that $T_{L}(u \gg 1)$ alone determines the zero-field susceptibility. We identify

$$
j=\rho_{0} J_{\mathrm{K}}=\frac{8}{\pi u}
$$

to arrive at $(u \gg 1, j \ll 1)$

$$
\begin{aligned}
\frac{\chi_{0}^{\mathrm{ii}, \operatorname{SIAM}}(u)}{\left(g_{e} \mu_{\mathrm{B}}\right)^{2}} & \approx \frac{\bar{\chi}_{0}(j)}{\left(g_{e} \mu_{\mathrm{B}}\right)^{2}} \frac{1}{\sqrt{\pi} U} e^{-\pi^{2} j / 16} \\
& =\frac{\bar{\chi}_{0}(j)}{\left(g_{e} \mu_{\mathrm{B}}\right)^{2}} s_{0}^{\mathrm{SIAM}}\left(1+\sum_{n=1}^{\infty} \frac{s_{n}^{\mathrm{SIAM}}}{s_{0}^{\operatorname{SIAM}}} j^{n}\right) .
\end{aligned}
$$

As for the SIKM, the impurity-induced zero-field susceptibility $\chi_{0}^{\mathrm{ii}, S I A M}(j)$ can be cast into the form (122). To reproduce the results for the SIKM from the SIAM for $u \gg 1$, we need to set $s_{0}^{\text {SIAM }}=s_{0}$ so that $U_{\mathrm{H}} \equiv 2 \mathcal{D} \sqrt{e}>W$ for a constant density of states which implies that $V$ or $\Gamma$ varies while $U_{\mathrm{H}}$ is fixed. 
Unfortunately, it is not possible to identify the coefficients $s_{n}$ with $s_{n}^{\text {SIAM }}$ because the formula (124) contains the contributions of the charge degrees of freedom that become relevant for $n \geqslant 1$. Therefore, the Bethe-ansatz results for the SIAM at strong coupling cannot be used for the SIKM beyond leading order.

\section{Impurity-induced magnetization}

The Bethe ansatz provides the impurity-induced magnetization of the system $m^{\mathrm{ii}}\left(J_{\mathrm{K}}^{\mathrm{BA}}, B, T\right)$ at finite temperatures $T$ and finite external fields $B$ [see Eq. (19)]. For small couplings $J_{\mathrm{K}} \ll 1$, the impurity-induced susceptibility is exponentially large [see Eq. (122)], so that the relevant magnetic fields that lead to a finite magnetization are exponentially small. The polarization of the host electrons becomes negligibly small, and we do not have to distinguish between the impurity spin polarization and the impurity-induced magnetization, i.e., $m^{\mathrm{ii}}\left(J_{\mathrm{K}} \ll 1, B \rightarrow 0\right)=m^{S}\left(J_{\mathrm{K}} \ll 1, B \rightarrow 0\right)$, with exponential accuracy. Therefore, the zero-field susceptibilities from the impurity-induced magnetization and from the impurity spin polarization become identical.

From Eqs. (5.1.34) and (5.1.37) in TW and (4.29) in AFL, the Bethe-ansatz result for the impurity-induced magnetization reads as $[e=\exp (1)]$

$$
\begin{gathered}
\frac{m^{\mathrm{ii}}(h \leqslant 1)}{g_{e} \mu_{\mathrm{B}}}=\frac{1}{2 \sqrt{\pi}} \sum_{n=0}^{\infty}\left(\frac{n+1 / 2}{e}\right)^{n+1 / 2} \frac{(-1)^{n} h^{2 n+1}}{n !(n+1 / 2)}, \\
\frac{m^{\mathrm{ii}}(h \geqslant 1)}{g_{e} \mu_{\mathrm{B}}}=\frac{1}{2}-\frac{1}{2 \pi^{3 / 2}} \int_{0}^{\infty} d \omega \frac{\sin (\pi \omega)}{\omega} \Gamma(1 / 2+\omega) \\
\times\left(\frac{\omega}{e}\right)^{-\omega} h^{-2 \omega} .
\end{gathered}
$$

In Eqs. (128) and (129), the external field is scaled by the universal low-temperature magnetic energy scale $T_{1}$ :

$$
\begin{aligned}
h & =B / T_{1}, \\
T_{1} & =\sqrt{\frac{2 \pi}{e}} T_{0}=\sqrt{\frac{2 \pi}{e}}\left(\frac{4 \pi \chi_{0}^{\mathrm{ii}}\left(J_{\mathrm{K}}\right)}{\left(g_{e} \mu_{\mathrm{B}}\right)^{2}}\right)^{-1} .
\end{aligned}
$$

Since $\chi_{0}^{\mathrm{ii}}\left(J_{\mathrm{K}}\right)$ can be calculated analytically in terms of $\rho_{0}(0) J_{\mathrm{K}}$ only to leading order [see Eq. (123)], we follow the usual approach and determine $T_{1}$ numerically from the zero-field susceptibility [13].

\section{NUMERICAL APPROACHES}

In this section, we briefly discuss two numerically exact approaches to the many-body problem. We begin with the density-matrix renormalization group (DMRG) method, and move on to the numerical renormalization group (NRG) technique that performs the Wilson renormalization scheme numerically.

\section{A. DMRG}

\section{Impurity spin polarization and impurity-induced magnetization}

a. Impurity spin polarization. When we apply the magnetic field only at the impurity, standard DMRG ground-state calculations provide the results for the impurity spin polarization $\left\langle\hat{S}^{z}\right\rangle=m_{\text {loc }}^{S} /\left(g_{e} \mu_{\mathrm{B}}\right)$. For a globally applied field $\mathcal{H}$ the calculation of $\left\langle\hat{S}^{z}\right\rangle$ is more subtle because the total spin in the $z$ direction $S_{\text {tot }}^{z}=S^{z}+s^{z}$ is a good quantum number [28] (see Sec. II B 2). Therefore, the spin quantum number $S_{\text {tot }}^{z}$ changes from $S_{\text {tot }}^{z}=0$ for $\mathcal{H}=0$ to $S_{\text {tot }}^{z}=1,2,3, \ldots$ for increasing external fields in steps of $g \mu_{\mathrm{B}} \mathcal{H}_{n}$ whenever

$$
g \mu_{\mathrm{B}} \mathcal{H}_{n}=E_{0}\left(S_{\text {tot }}^{z}=n\right)-E_{0}\left(S_{\text {tot }}^{z}=n-1\right)
$$

for $n=1,2,3, \ldots$. Thus, the impurity spin polarization is recorded only at discrete values of the external field whereby expectation values are calculated with the ground state for $S_{\text {tot }}^{z}=n$. For $J_{\mathrm{K}} \leqslant 1$, we use system sizes $L=$ $29,61,125,253,509,637,765$. Since this approach hampers a systematic finite-size extrapolation, we plot $m^{S}(B) /\left(g_{e} \mu_{\mathrm{B}}\right)$ for our largest system sizes.

For $J_{\mathrm{K}} \gtrsim 1$ and a global magnetic field, the calculation of the impurity spin polarization faces the problem that the impurity and the electron spin at $n=0$ form a singlet and tend to separate from the rest of the system. This reduces the effective length of the half-chain by one site, and a finite-size gap opens at the Fermi energy. To counteract this effect for $J_{\mathrm{K}} \gtrsim 1$, we subtract two sites from the original chain, i.e., we use $L=27,59,123,251,507,635,763$. Then, the ground state at $\mathcal{H}=0^{+}$has total spin $S_{\text {tot }}^{z}=\frac{1}{2}$, and the impurity magnetization of the ground states at $S_{\text {tot }}^{z}=n+\frac{1}{2}$ $(n=1,2,3, \ldots)$ is recorded.

b. Impurity-induced magnetization. In DMRG, we can calculate the ground-state energy $E_{0}\left(J_{\mathrm{K}}, S_{\text {tot }}^{z}, L\right)$ for given integer $0 \leqslant S_{\text {tot }}^{z} \leqslant(L+3) / 2$. For very large system sizes, $\Delta E\left(J_{\mathrm{K}}, S_{\mathrm{tot}}^{z}, L\right)=E_{0}\left(J_{\mathrm{K}}, S_{\mathrm{tot}}^{z}, L\right)-E_{0}\left(J_{\mathrm{K}}, 0, L\right)$ can be fitted to a positive, continuous function of $s \equiv S_{\text {tot }}^{z}$. Then, the global external field is obtained from

$$
B=\frac{\partial \Delta E\left(J_{\mathrm{K}}, s, L\right)}{\partial s} \equiv E_{0}^{\prime}(s) .
$$

In turn, we may solve Eq. (132) for the total spin $s_{\text {opt }}(B)$ as a function of $B$,

$$
s_{\mathrm{opt}}(B)=\left[E_{0}^{\prime}\right]^{-1}(B),
$$

where $\left[E_{0}^{\prime}\right]^{-1}(x)$ is the inverse function of $E_{0}^{\prime}(x)$ for given $J_{\mathrm{K}}$ and $L$. Thus, the impurity-induced magnetization for a global field is given by

$$
\frac{m^{\mathrm{ii}, \mathrm{DMRG}}\left(J_{\mathrm{K}}, B, L\right)}{g_{e} \mu_{\mathrm{B}}}=s_{\mathrm{opt}}\left(J_{\mathrm{K}}, B, L\right)-s_{\mathrm{opt}}^{\mathrm{free}}(B, L) .
$$

For a local field, one has to also calculate the impurity spin polarization $\left\langle\hat{S}^{z}\right\rangle$ as a function of $S_{\text {tot }}^{z}$.

In practice, it requires exceedingly large system sizes to carry out this program because, in the region of small Kondo couplings $J_{\mathrm{K}} \lesssim 0.5$, the susceptibility is very large so that the system is almost fully polarized for very small fields even for system sizes $L=\mathcal{O}\left(10^{3}\right)$. For this reason the analytic curve $E_{0}(s)$ is not known with the required accuracy. Therefore, we do not employ the DMRG to calculate impurity-induced quantities.

\section{Technicalities}

The accuracy of the calculations is controlled using the dynamic block-state selection (DBSS) scheme [33,34]. Setting 

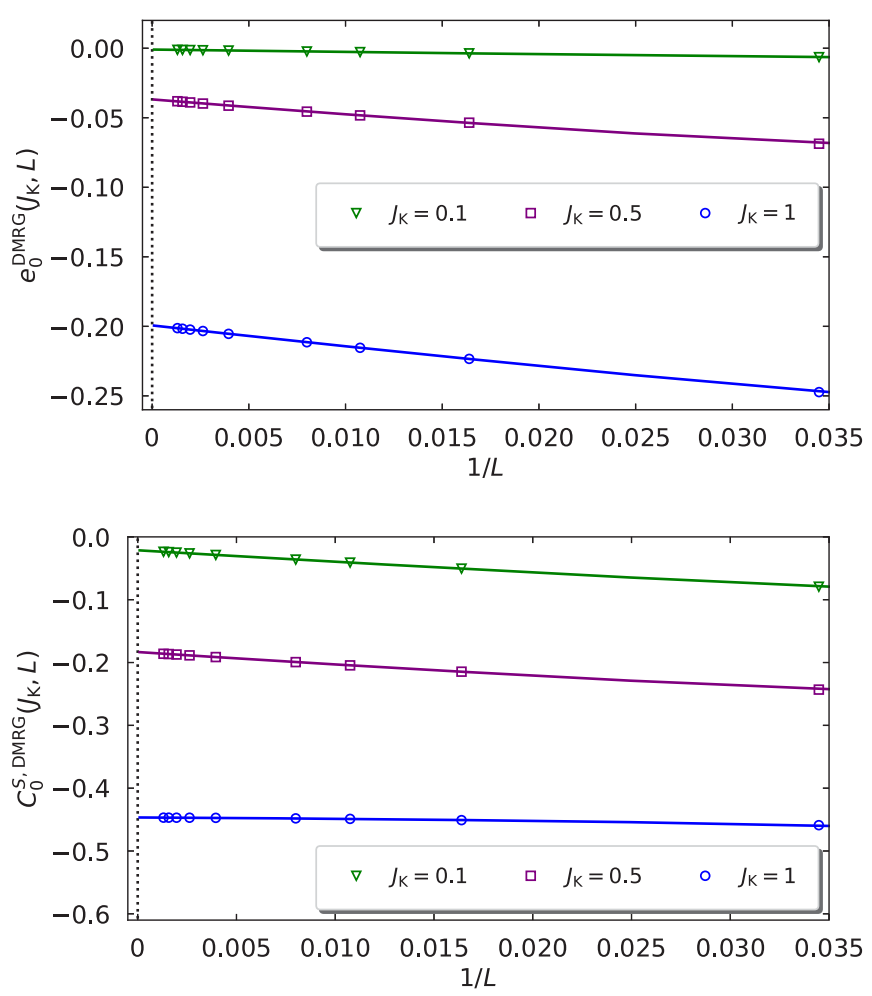

FIG. 3. Ground-state energy $e_{0}^{\mathrm{DMRG}}\left(J_{\mathrm{K}}, L\right)$ from DMRG (upper figure) and local spin correlation function $C_{0}^{S, \mathrm{DMRG}}\left(J_{\mathrm{K}}, L\right)$ (lower figure) of the one-dimensional symmetric Kondo model as a function of inverse system size $1 / L$ for $J_{\mathrm{K}}=0.1,0.5,1$. The solid lines represent the second-order polynomial fit in $1 / L$.

the control parameter to $\chi=10^{-5}$, the truncation error yields around $10^{-7}$ while the number of maximally kept DMRG block states was observed to in the range $M=5000$ for our largest system sizes.

We use DMRG to calculate the excess ground-state energy $e_{0}\left(J_{\mathrm{K}}, L\right)$ [see Eq. (9)] and extrapolate to the thermodynamic limit

$$
e_{0}^{\mathrm{DMRG}}\left(J_{\mathrm{K}}\right)=\lim _{L \rightarrow \infty} e_{0}^{\mathrm{DMRG}}\left(J_{\mathrm{K}}, L\right)
$$

using a second-order polynomial fit in $1 / L$. As an example, we present the ground-state energy $e_{0}^{\mathrm{DMRG}}\left(J_{\mathrm{K}}, L\right)$ and the local spin correlation $C_{0}^{S, \text { DMRG }}\left(J_{\mathrm{K}}, L\right)$ as a function of inverse system size for $J_{\mathrm{K}}=0.1,0.5,1$ in Fig. 3; the finite-size extrapolation is unproblematic.

In Fig. 4 we show the zero-field impurity spin susceptibility $\chi_{0}^{\mathrm{S}, \text { DMRG }}\left(J_{\mathrm{K}}, L\right)$ for a global magnetic field and $\chi_{0, \text { loc }}^{\text {S,DMRG }}\left(J_{\mathrm{K}}, L\right)$ for a local magnetic field on a logarithmic scale as a function of inverse system size $1 / L$ for $J_{\mathrm{K}}=$ $0.6,1,5$. Apparently, the finite-size extrapolation can safely be performed for the zero-field impurity spin susceptibility for moderate to large coupling strengths, $J_{\mathrm{K}} \gtrsim 0.6$, because the NRG data are reasonably well reproduced.

As in the case of the single-impurity Anderson model [28], the DMRG calculations for a global magnetic field are troubled for small Kondo couplings. This is shown in Fig. 5 for $J_{\mathrm{K}}=0.4,0.5,0.6$. For $J_{\mathrm{K}} \ll 1$, it requires exponentially increasing system sizes to resolve the exponentially small
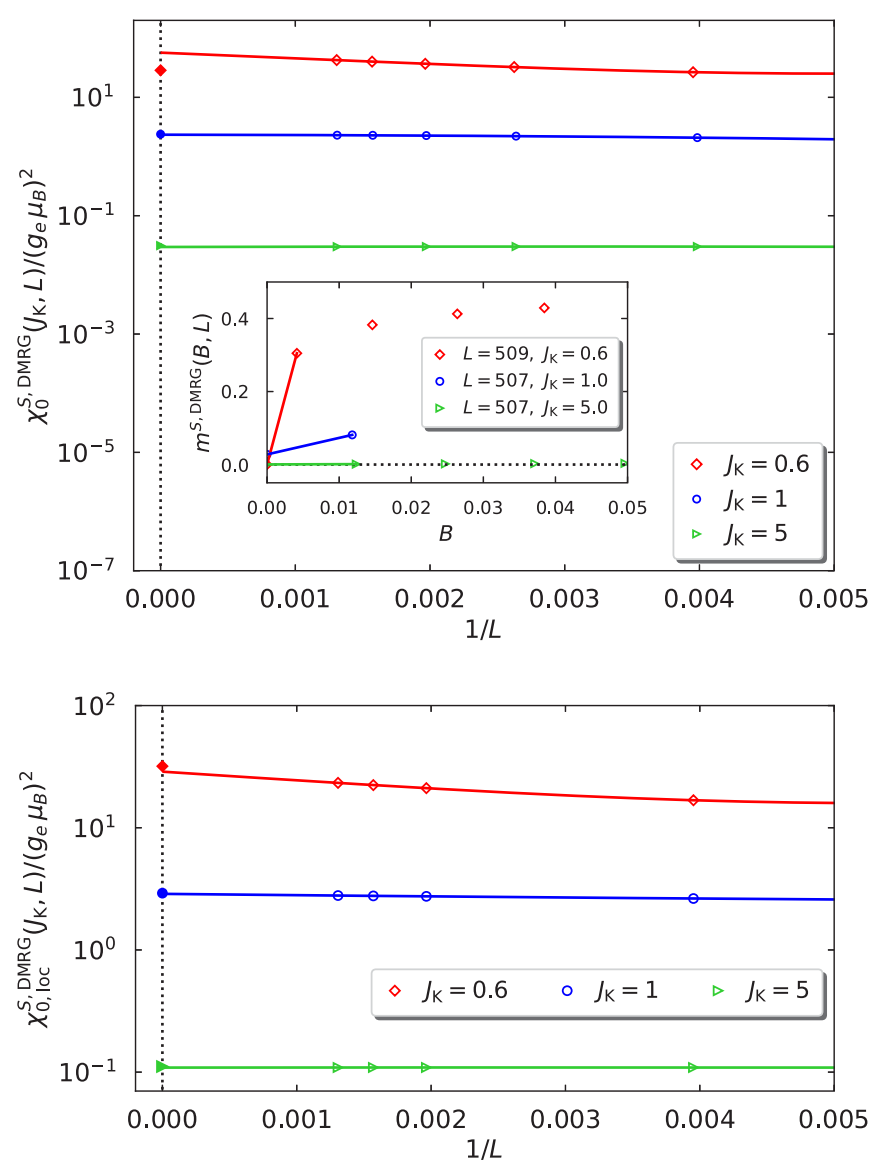

FIG. 4. Zero-field impurity spin susceptibility $\chi_{0}^{S \text {,DMRG }}\left(J_{\mathrm{K}}, L\right)$ from DMRG for a global magnetic field (upper figure) and $\chi_{0, \text { loc }}^{S, \text { DMRG }}\left(J_{\mathrm{K}}, L\right)$ for a local magnetic field (lower figure) on a logarithmic scale for the one-dimensional symmetric Kondo model as a function of inverse system size $1 / L$ for $J_{\mathrm{K}}=0.6,1,5$. For comparison, data from NRG are shown as filled symbols at $1 / L=$ 0 . The solid lines represent the second-order polynomial fit in $1 / L$ on the logarithm of the susceptibility. The inset shows the magnetization $m^{S, \text { DMRG }}\left(J_{\mathrm{K}}, B, L=507,509\right)$ as a function of a small applied field whose slope defines $\chi_{0}^{S, \text { DMRG }}\left(J_{\mathrm{K}}, L\right) /\left(g_{e} \mu_{\mathrm{B}}\right)^{2} \approx$ $\left[m^{S, \text { DMRG }}\left(J_{\mathrm{K}}, B_{1}, L\right)-m^{S, \text { DMRG }}\left(J_{\mathrm{K}}, 0, L\right)\right] /\left(2 B_{1}\right)$.

energy scale for spin excitations, i.e., for $J_{\mathrm{K}} \lesssim 0.5$, a reliable extrapolation of the susceptibility to the thermodynamic limit requires system sizes that already exceed $L=10^{3}$ by far. For a local magnetic field, the DMRG can access low fields so that the zero-field impurity spin susceptibility is much better behaved at small interactions. Nevertheless, the extrapolation is not very stable, as can be seen from the winding fitting curves, and the NRG values cannot be recovered faithfully. Again, for $J_{\mathrm{K}} \lesssim 0.5$ a reliable extrapolation of the DMRG values for the zero-field impurity spin susceptibility to the thermodynamic limit requires exponentially large system sizes.

\section{B. NRG}

Here, we compile basic information about the NRG algorithm that we employ in our work; for a review on NRG, see Ref. [8]. 

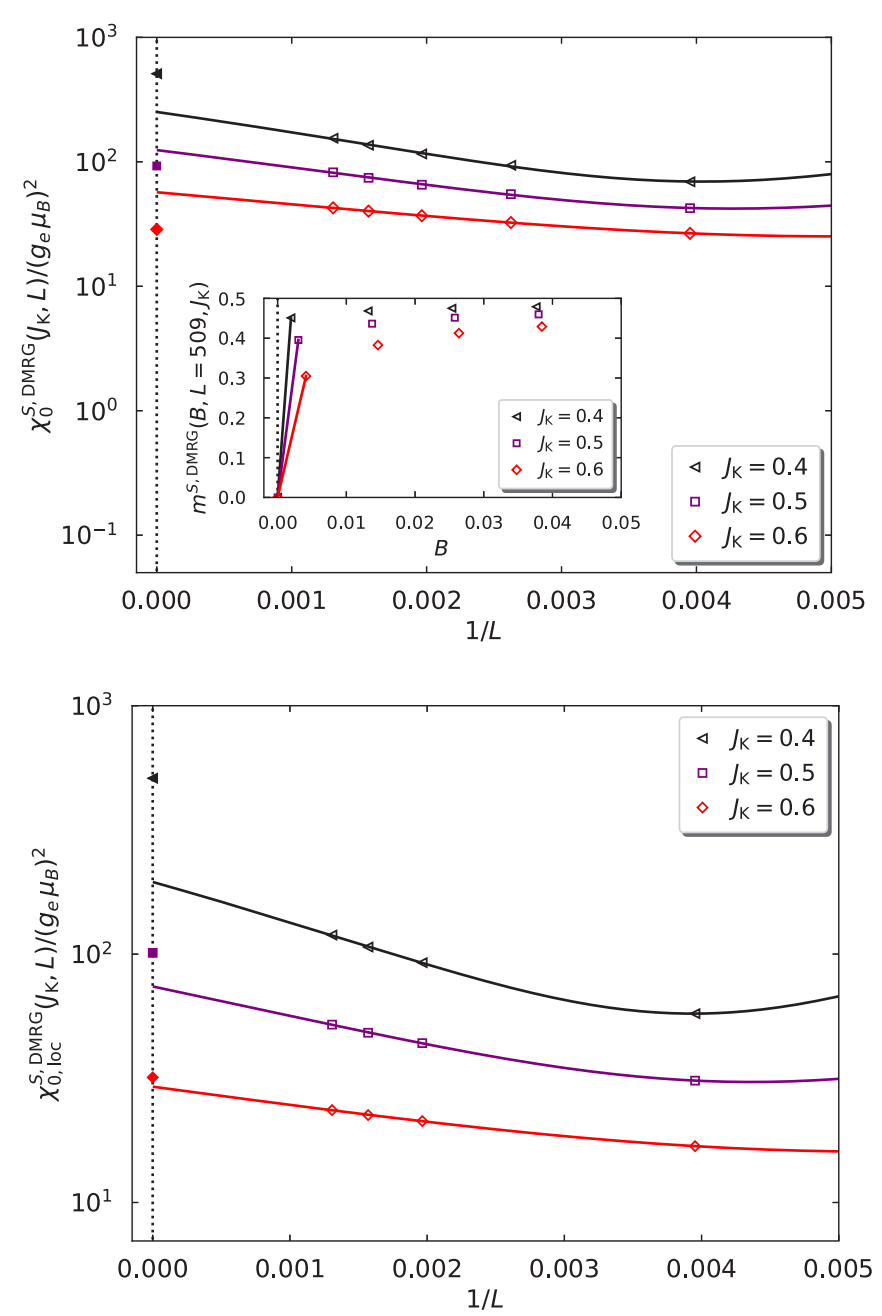

FIG. 5. Same content as Fig. 4 but for $J_{\mathrm{K}}=0.4,0.5,0.6$. Inset: magnetization $m^{S, \text { DMRG }}\left(J_{\mathrm{K}}, B, L=509\right)$ as a function of a small applied field for $J_{\mathrm{K}}=0.4,0.5,0.6$ whose slope defines $\chi_{0}^{S, \mathrm{DMRG}} /\left(g_{e} \mu_{\mathrm{B}}\right)^{2}$.

\section{Wilson chain}

The NRG starts from the energy representation of the Hamiltonian [13]

$$
\hat{H}_{\mathrm{K}}=\hat{T}^{\mathrm{NRG}}+\hat{V}_{\mathrm{sd}}^{\mathrm{NRG}}+\hat{H}_{\mathrm{loc}, \mathrm{m}}
$$

with the kinetic energy

$$
\hat{T}^{\mathrm{NRG}}=\sum_{\sigma} \int_{-1-\sigma_{n} B}^{1-\sigma_{n} B} d \epsilon \epsilon \tilde{a}_{\epsilon, \sigma}^{+} \tilde{a}_{\epsilon, \sigma}
$$

and the local Kondo interaction

$$
\begin{aligned}
\hat{V}_{\mathrm{sd}}^{\mathrm{NRG}}= & \frac{J_{\mathrm{K}}}{2}\left(\hat{f}_{0, \uparrow}^{+} \hat{f}_{0, \downarrow} \hat{d}_{\downarrow}^{+} \hat{d}_{\uparrow}+\hat{f}_{0, \downarrow}^{+} \hat{f}_{0, \uparrow} \hat{d}_{\uparrow}^{+} \hat{d}_{\downarrow}\right) \\
& +\frac{J_{\mathrm{K}}}{4}\left(\hat{d}_{\uparrow}^{+} \hat{d}_{\uparrow}-\hat{d}_{\downarrow}^{+} \hat{d}_{\downarrow}\right)\left(\hat{f}_{0, \uparrow}^{+} \hat{f}_{0, \uparrow}-\hat{f}_{0, \downarrow}^{+} \hat{f}_{0, \downarrow}\right) .
\end{aligned}
$$

Here, the electron mode that couples to the impurity is given by

$$
\hat{f}_{0, \sigma}=\int_{-1-\sigma_{n} B}^{1-\sigma_{n} B} d \epsilon \sqrt{\rho_{0}\left(\epsilon+\sigma_{n} B\right)} \tilde{a}_{\epsilon, \sigma}
$$

where $\sigma_{n}=1$ for $\sigma=\uparrow$ and $\sigma_{n}=-1$ for $\sigma=\downarrow$. In this step, no approximation is introduced.

The decisive step is the logarithmic discretization of the NRG Hamiltonian (138). In the presence of a global field, the upper and lower band edges differ from each other

$$
W_{ \pm, \sigma}= \pm 1-\sigma_{n} B \text {. }
$$

Thus, we follow Hager [8,35] and define the sampling points

$$
x_{n, \sigma, \pm}=W_{ \pm, \sigma} \Lambda^{-n}, \quad n=0,1,2, \ldots
$$

that depend on the position of the upper $(+)$ and lower $(-)$ band edges for spin $\sigma=\uparrow, \downarrow$. As usual [13], we approximate the density of states in each interval $I_{n, \sigma}^{+}=\left[x_{n+1, \sigma,+}, x_{n, \sigma,+}\right]$ and $I_{n, \sigma}^{-}=\left[x_{n, \sigma,-}, x_{n+1, \sigma,-}\right]$ by a suitably chosen constant. With the interval width

$$
d_{n, \sigma}^{ \pm}=\left|W_{ \pm, \sigma}\right| \Lambda^{-n}\left(1-\Lambda^{-1}\right)
$$

we define

$$
\left(\gamma_{n, \sigma}^{ \pm}\right)^{2}=\int_{I_{n, \sigma}^{ \pm}} d \epsilon \rho_{0}\left(\epsilon+\sigma_{n} B\right)
$$

and the expansion operators

$$
\tilde{b}_{n, p, \sigma, \pm}=\int_{I_{n, \sigma}^{ \pm}} d \epsilon \frac{1}{\sqrt{d_{n}^{ \pm}}} e^{\mp 2 \pi i p \epsilon / d_{n}^{ \pm}} \tilde{a}_{\epsilon, \sigma}
$$

such that we can write

$$
\hat{f}_{0, \sigma}=\sum_{n}\left(\gamma_{n, \sigma}^{+} \tilde{b}_{n, 0, \sigma,+}+\gamma_{n, \sigma}^{-} \tilde{b}_{n, 0, \sigma,-}\right)
$$

for the bath state that couples to the impurity. Note that only the mode $p=0$ appears in the bath-electron operator $\hat{f}_{0, \sigma}$.

The kinetic energy becomes

$$
\begin{aligned}
\hat{T}^{\mathrm{NRG}}= & \sum_{n, p, p^{\prime}, \sigma} \zeta_{n, p, p^{\prime}, \sigma}^{+} \tilde{b}_{n, p, \sigma,+}^{+} \tilde{b}_{n, p^{\prime}, \sigma,+} \\
& +\zeta_{n, p, p^{\prime}, \sigma}^{-} \tilde{b}_{n, p, \sigma,-}^{+} \tilde{b}_{n, p^{\prime}, \sigma,-}
\end{aligned}
$$

To construct the Wilson chain we now drop all modes $p \neq 0$ in the kinetic energy

$$
\hat{T}^{\mathrm{NRG}} \approx \sum_{n, \sigma} \zeta_{n, \sigma}^{+} \tilde{b}_{n, \sigma,+}^{+} \tilde{b}_{n, \sigma,+}+\zeta_{n, \sigma}^{-} \tilde{b}_{n, \sigma,-}^{+} \tilde{b}_{n, \sigma,-}
$$

with $\tilde{b}_{n, \sigma, \pm} \equiv \tilde{b}_{n, 0, \sigma, \pm}$ and

$$
\zeta_{n, \sigma}^{ \pm} \equiv \zeta_{n, 0,0, \sigma}^{ \pm}=\frac{\int_{I_{n, \sigma}^{ \pm}} d \epsilon \epsilon \rho_{0}\left(\epsilon+\sigma_{n} B\right)}{\int_{I_{n, \sigma}^{ \pm}} d \epsilon \rho_{0}\left(\epsilon+\sigma_{n} B\right)} .
$$

This approximation becomes exact in the limit $\Lambda \rightarrow 1$; for a thorough discussion, see Ref. [8].

As a final step in the construction of the Wilson chain, we choose $\left|0_{\sigma}\right\rangle \equiv \hat{f}_{0, \sigma}^{+} \mid$vac $\rangle$as starting vector for the iterative construction of the Lanczos vectors (see Appendix B 2). The kinetic energy operator is then represented as a tight-binding Hamiltonian on a chain:

$$
\hat{T}^{\mathrm{NRG}}=\sum_{n=0, \sigma}^{\infty} \varepsilon_{n, \sigma} \hat{f}_{n, \sigma}^{+} \hat{f}_{n, \sigma}+t_{n, \sigma}\left(\hat{f}_{n, \sigma}^{+} \hat{f}_{n+1, \sigma}+\text { H.c. }\right)
$$

The matrix elements are calculated according to Eqs. (27)(31) in Ref. [8]. For completeness, we give the details in Appendix A 9. 


\section{Technicalities}

The Wilson chain is solved iteratively, as described in detail in Ref. [8]. As maximal chain length we use $30 \leqslant n_{\max } \leqslant$ 100 , depending on the value of $J_{\mathrm{K}}$ and the discretization parameter $\Lambda$. At the end of each diagonalization step in the renormalization group procedure, we keep $3000<N_{s}<5000$ lowest-energy eigenstates.

At the end of the renormalization group calculation after adding the next Wilson site, we thus have $4 N_{s}$ states with their global quantum numbers (energy, particle number, spin component in $z$ direction) that permit the calculation of thermodynamic quantities such as the ground state and free energy, impurity-induced magnetization, and magnetic susceptibility by taking the derivative with respect to the external field [see Eq. (21)]. For large couplings $J_{\mathrm{K}} \gtrsim 5$, it is numerically advantageous to calculate the zero-field impurityinduced susceptibility from the second-order derivative of the ground-state energy.

For the calculation of local expectation values, e.g., the local spin correlation and impurity spin polarization, the corresponding quantities are expressed in terms of the Wilson chain operators and are transformed in each renormalization group step.

The discretization parameter $\Lambda$ we choose in the range $1.8 \leqslant \Lambda \leqslant 3.2$. To include the discretization correction for reconnecting with the original continuum model even for a finite $\Lambda$, we follow Krishna-murthy, Wilkins, and Wilson $[6,7]$, and multiply the Kondo coupling with the correction factor

$$
A_{\Lambda}(\Lambda)=\frac{1}{2} \frac{\Lambda+1}{\Lambda-1} \ln (\Lambda)
$$

that becomes unity for $\Lambda \rightarrow 1$. The factor was derived for a constant density of states, but we shall see that it also works very well for the one-dimensional density of states.

As an example, we consider the ground-state energy. We calculate $e_{0}^{\mathrm{NRG}}\left(J_{\mathrm{K}}, \Lambda\right)$ and extrapolate to the limit $\Lambda \rightarrow 1$,

$$
e_{0}^{\mathrm{NRG}}\left(J_{\mathrm{K}}\right)=\lim _{\Lambda \rightarrow 1} e_{0}^{\mathrm{NRG}}\left(J_{\mathrm{K}}, \Lambda\right),
$$

using a second-order polynomial fit in $(\Lambda-1)$. In Fig. 6 we present the ground-state energy $e_{0}^{\mathrm{NRG}}\left(J_{\mathrm{K}}, \Lambda\right)$ and the local spin correlation $C_{0}^{S, \mathrm{NRG}}\left(J_{\mathrm{K}}, \Lambda\right)$ as a function of the Wilson parameter $\Lambda$ for $J_{\mathrm{K}}=0.1,0.5,1$.

The extrapolation to $\Lambda \rightarrow 1$ provides very good results in comparison with DMRG. Note that it requires $\Lambda$ values as small as $\Lambda=1.8$ to achieve an agreement of the extrapolated $\mathrm{NRG}$ values and DMRG data within an accuracy of better than $1 \%$.

For an independent assessment of the quality of the $\Lambda$ extrapolation, we also performed NRG calculations where we switched off the correction factor $A_{\Lambda}(\Lambda)$. Recall that the correction factor was derived for a constant density of states and thus does not necessarily perform perfectly for the one-dimensional density of states. As seen from Fig. 7, the extrapolated values for the ground-state energy differ by less than $1 \%$. In particular, the resulting energy values are slightly below the DMRG values when the correction factor is switched off whereas they remain consistently above the DMRG energies when the correction factor is employed.
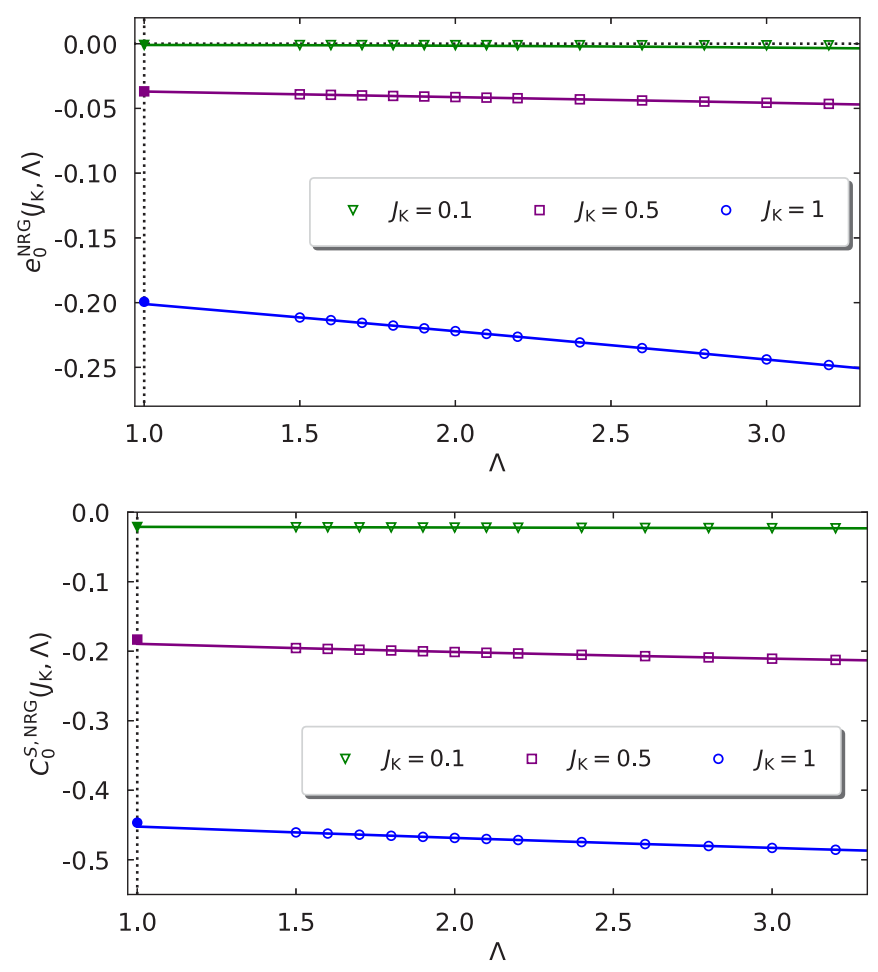

FIG. 6. Ground-state energy $e_{0}^{\mathrm{NRG}}\left(J_{\mathrm{K}}, \Lambda\right)$ from NRG (upper figure) and local spin correlation function $C_{0}^{S, \mathrm{NRG}}\left(J_{\mathrm{K}}, \Lambda\right)$ (lower figure) of the one-dimensional symmetric Kondo model as a function of the Wilson parameter $\Lambda$ for $J_{\mathrm{K}}=0.1,0.5,1$. The solid lines represent a second-order polynomial fit in $(\Lambda-1)$. Filled symbols at $\Lambda=1$ represent the DMRG values.

Therefore, we keep the correction factor in all our NRG calculations.

In Fig. 8 we show the zero-field impurity spin susceptibility $\chi_{0}^{S, \mathrm{NRG}}\left(J_{\mathrm{K}}, \Lambda\right)$ for a global magnetic field and $\chi_{0, \mathrm{loc}}^{S, \mathrm{NRG}}\left(J_{\mathrm{K}}, \Lambda\right)$ for a local magnetic field as a function of $\Lambda$ for $J_{\mathrm{K}}=$ $0.3,0.4,0.6,1,5$. In contrast to DMRG, the extrapolation

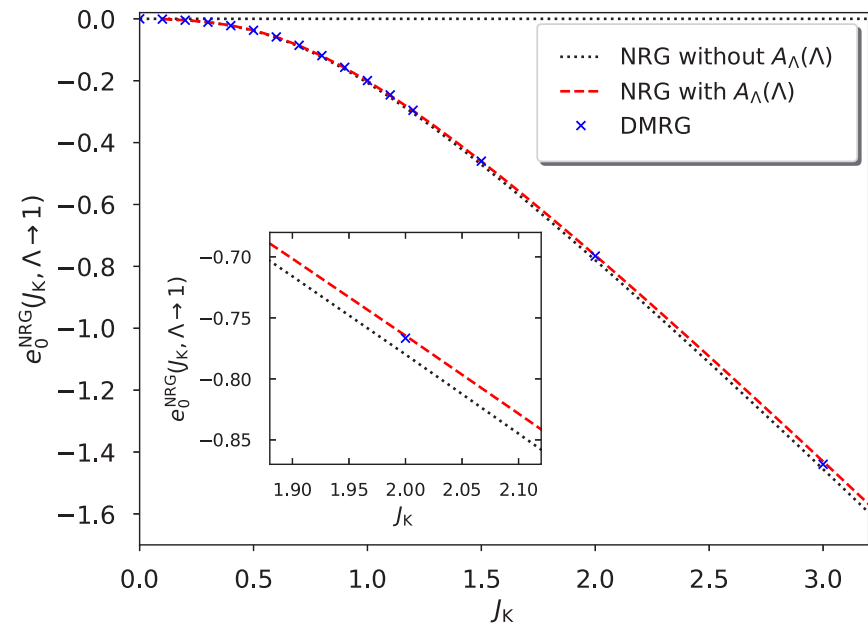

FIG. 7. Ground-state energy $e_{0}^{\mathrm{NRG}}\left(J_{\mathrm{K}}, \Lambda\right)$ from NRG of the onedimensional symmetric Kondo model as a function of the Kondo coupling $J_{\mathrm{K}}$. NRG data with and without the correction factor $A_{\Lambda}(\Lambda)$ were extrapolated to $\Lambda \rightarrow 1$. DMRG data are shown for comparison. 

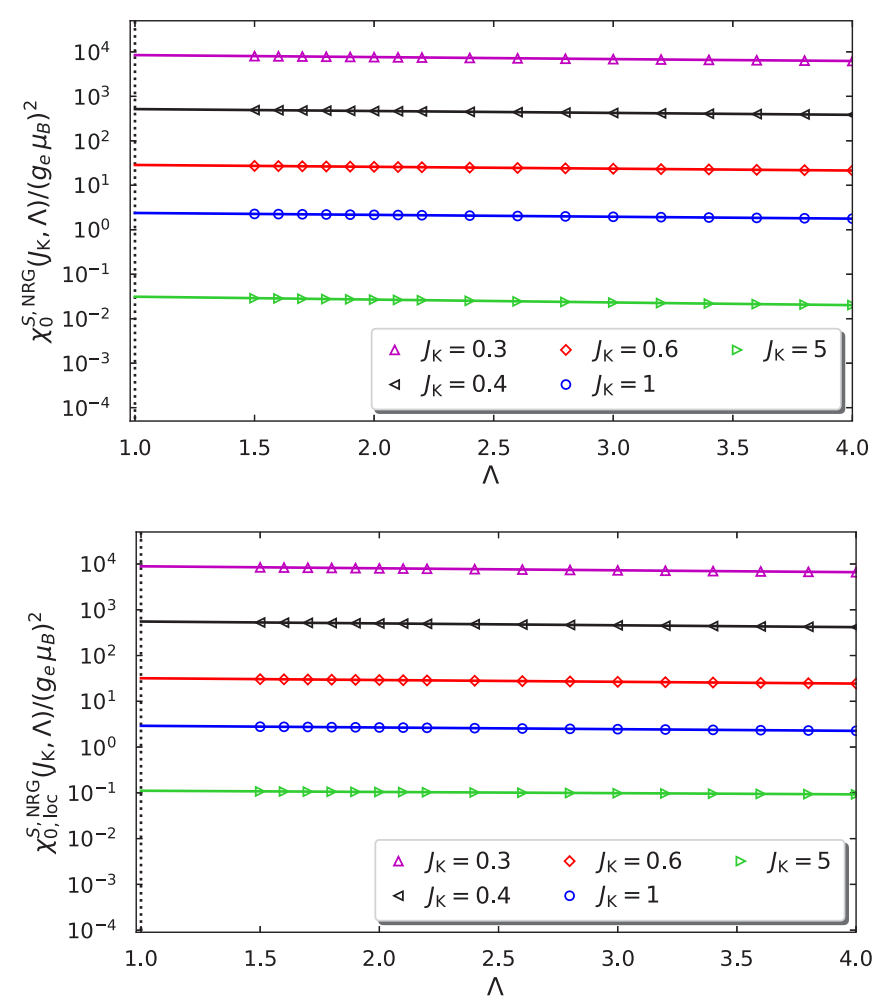

FIG. 8. Zero-field impurity spin susceptibility $\chi_{0}^{S, \mathrm{NRG}}\left(J_{\mathrm{K}}, \Lambda\right)$ from NRG for a global magnetic field (upper figure) and $\chi_{0, \text { loc }}^{S, N R G}\left(J_{K}, \Lambda\right)$ for a local magnetic field (lower figure) for the onedimensional symmetric Kondo model as a function of the Wilson parameter $\Lambda$ for $J_{\mathrm{K}}=0.3,0.4,0.6,1,5$. The solid lines represent the second-order polynomial fit of $\ln \left[\chi_{0}(\Lambda)\right]$ in $(\Lambda-1)$.

of the NRG data can safely be performed for the zero-field impurity spin susceptibility for all coupling strengths.

\section{COMPARISON}

We begin our comparison with the ground-state energy and the local spin correlation. Next, we compare the zerofield susceptibilities, and the impurity spin polarization and impurity-induced magnetization.

\section{A. Ground-state energy and local spin correlation}

\section{Ground-state energy at small Kondo couplings}

In Fig. 9 we show the ground-state energy for small Kondo couplings $J_{\mathrm{K}} \leqslant 0.4$. In this parameter region, the Yosida and paramagnetic Gutzwiller energies are exponentially small which results in a poor variational energy bound for $J_{\mathrm{K}} \leqslant 0.4$. Therefore, we do not display them here.

The Lanczos approach displays the correct quadratic dependence of the ground-state energy on $J_{\mathrm{K}}$. However, the prefactor is too small by a factor $\pi / 4$ [see Eqs. (34) and (51)]. The best analytic variational bound is provided by the magnetically ordered Gutzwiller state. As seen from Eq. (A82), it reproduces $96.5 \%$ of the second-order perturbation energy term, and gives a very good approximation for the groundstate energy for weak couplings. Note, however, that the exact solution has $m=0$ at $B=0^{+}$, i.e., the magnetic Gutzwiller

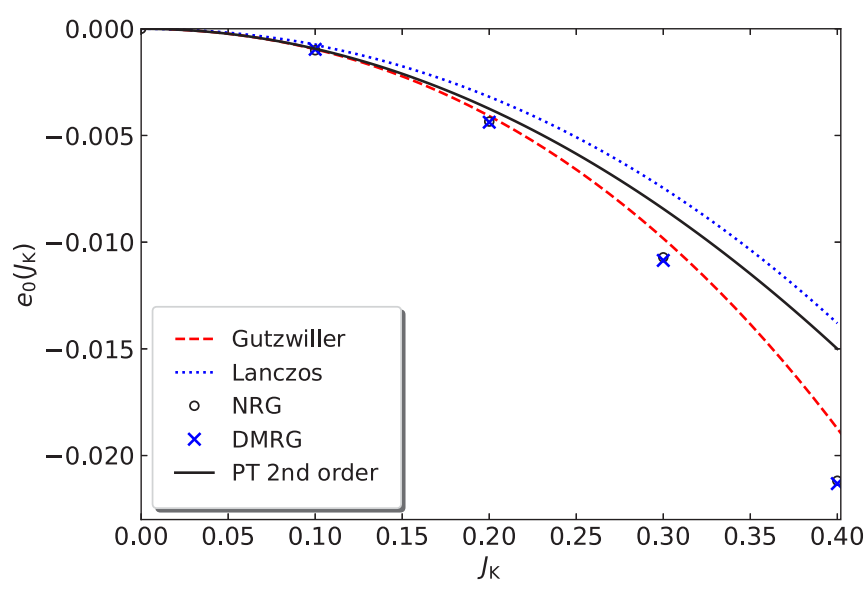

FIG. 9. Ground-state energy of the one-dimensional symmetric single-impurity Kondo model as a function of the Kondo coupling for small couplings $0 \leqslant J_{\mathrm{K}} \leqslant 0.4$. We compare results from firstorder Lanczos approximation (blue dotted line) [Eq. (50)], magnetic Gutzwiller theory (red dashed line) [Eq. (A82)], perturbation theory to second order (black full line) [Eq. (34)], and numerical data from DMRG (blue crosses) and NRG (black circles). Not shown are the exponentially small Yosida and paramagnetic Gutzwiller energies.

state with $m^{\mathrm{G}}\left(B=0^{+}\right)>0$ does not describe the ground-state physics correctly.

The NRG and DMRG energies differ by not more than $1 \%$, and thus provide independent and accurate values for the ground-state energy. As seen from Fig. 9, the quadratic approximation to the exact ground-state energy holds up to $J_{\mathrm{K}} \approx 0.1$, beyond which cubic and quartic terms in $J_{\mathrm{K}}$ become discernible.

\section{Ground-state energy at intermediate and large Kondo couplings}

In Fig. 10 we show the ground-state energy for intermediate to large Kondo couplings $0.4 \leqslant J_{\mathrm{K}} \leqslant 3.2$; recall that $W=2$ is the bandwidth of the host electrons. Again, the NRG and DMRG data lie essentially on top of each other and thus provide independent and accurate values for the ground-state energy. They converge to the strong-coupling estimate (42) for $e_{0}\left(J_{\mathrm{K}}\right)$.

Neither the Yosida wave function nor the first-order Lanczos state become asymptotically exact for strong couplings [see Eqs. (52) and (64)]. The best analytical variational upper bound results from the Gutzwiller state that displays no local symmetry breaking above $J_{\mathrm{K}, \mathrm{c}}^{\mathrm{G}} \approx 0.839$. In fact, as seen in Fig. 10, the Gutzwiller energy for strong coupling (94) is in excellent agreement with the NRG and DMRG data down to $J_{\mathrm{K}} \approx 1$, with deviations below $1 \%$. Therefore, we argue that the asymptotic expression (94) is exact up to and including second order in $1 / J_{\mathrm{K}}$.

\section{Local spin correlation}

In Fig. 11 we show the local spin correlation function $C_{0}^{S}\left(J_{\mathrm{K}}\right)$ as a function of the Kondo coupling $J_{\mathrm{K}}$. It is zero at $J_{\mathrm{K}}=0$ and decreases linearly for small interactions $C_{0}^{S}\left(J_{\mathrm{K}} \ll\right.$ $1)=-3 J_{\mathrm{K}} / 16$ [see Eq. (35)]. For large interactions, it reaches its limiting value, $C_{0}^{S}\left(J_{\mathrm{K}} \gg 1\right)=-\frac{3}{4}$ [see Eq. (43)], which corresponds to a singlet formed by the impurity spin and 


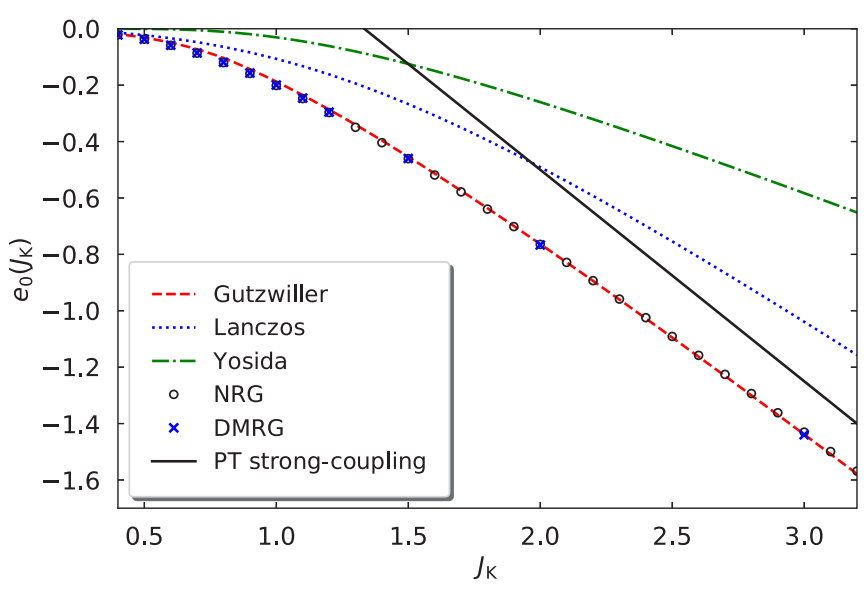

FIG. 10. Ground-state energy of the one-dimensional symmetric single-impurity Kondo model as a function of the Kondo coupling for intermediate and large couplings $0.4 \leqslant J_{\mathrm{K}} \leqslant 3.2$. We compare results from strong-coupling perturbation theory (black full line) [Eq. (42)], Gutzwiller theory (red dashed line), first-order Lanczos approximation (blue dotted line) [Eq. (50)], Yosida theory (green dotted-dashed line) [Eq. (58)], and numerical data from DMRG (blue crosses) and NRG (black circles).

a localized host electron. The DMRG and NRG data give the local spin correlation for all interaction strengths, and faithfully interpolate between the two limiting cases. We verified numerically that the Hellmann-Feynman theorem (12) is fulfilled both in DMRG and NRG.

While the Yosida and first-order Lanczos states are insufficient and thus omitted from the figure, the Gutzwiller wave function reproduces the numerical data for all interactions. The Gutzwiller state with $m>0$ for $J<J_{\mathrm{K}, \mathrm{c}}^{\mathrm{G}} \approx 0.839$ provides a quantitatively satisfactory value for the local spin correlation function but fails qualitatively because the exact solution does not sustain a locally symmetry-broken state. For $J>J_{\mathrm{K}, \mathrm{c}}^{\mathrm{G}}$, the Gutzwiller state very well approximates

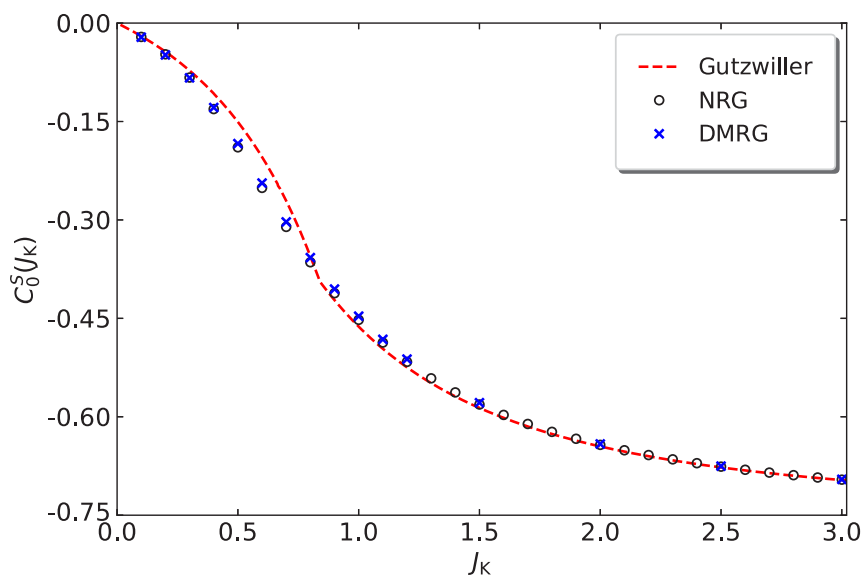

FIG. 11. Local spin correlation function $C_{0}^{S}\left(J_{\mathrm{K}}\right)$ of the onedimensional symmetric single-impurity Kondo model as a function of the Kondo coupling $J_{\mathrm{K}}$. We compare results from Gutzwiller theory (red dashed line) [Eq. (87)] and numerical data from DMRG (blue crosses) and NRG (black circles). The linear behavior for small couplings $C_{0}^{S}=-3 J_{\mathrm{K}} / 16$ is given by Eq. (35), the limiting value for strong couplings $C_{0}^{S}\left(J_{\mathrm{K}} \rightarrow \infty\right)=-\frac{3}{4}$ is found from Eq. (43). the local spin correlation function. As seen in Fig. 11, the Gutzwiller, DMRG, NRG results lie almost on top of each other for large Kondo couplings. Therefore, we argue that the strong-coupling expression (95) is actually exact up to and including third order in $1 / J_{\mathrm{K}}$.

\section{B. Magnetic susceptibilities for weak coupling}

For the zero-field susceptibilities, only the NRG is capable to examine the weak-coupling limit $J_{\mathrm{K}} \ll 1$, with the desired high accuracy. We mostly investigate the case of a constant density of states $\rho_{0}^{\text {const }}(0)=\frac{1}{2}$, for which the correction term $A_{\Lambda}(\Lambda)$ in Eq. (149) was originally derived [6,7]. For a onedimensional density of states, we show numerically that the ratio of the impurity-induced susceptibilities is given by the regularized first negative moment of the density of states (60).

\section{Impurity-induced magnetic susceptibility}

In Fig. 12 we show the zero-field impurity-induced magnetic susceptibility as a function of $J_{\mathrm{K}}$ for various values of
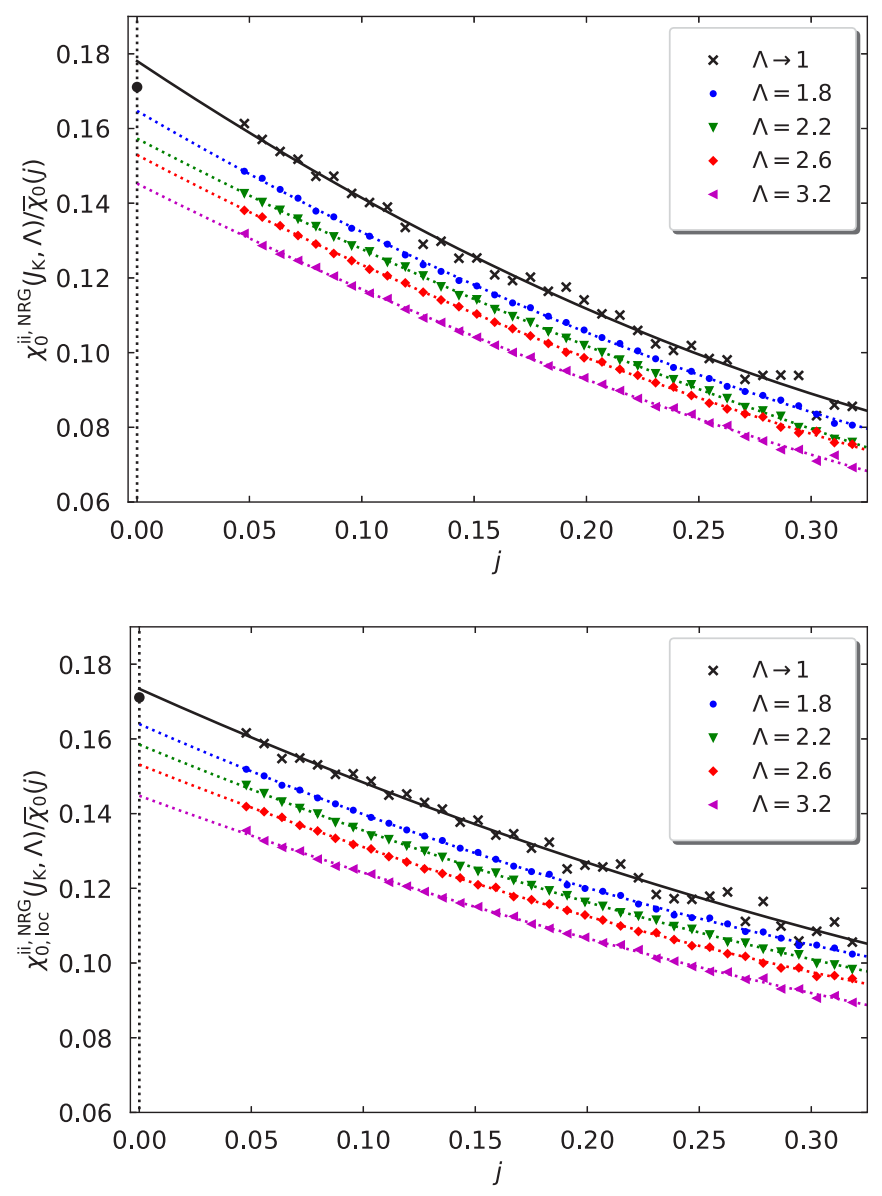

FIG. 12. Impurity-induced magnetic susceptibility $\chi_{0}^{\mathrm{ii}, \mathrm{NRG}}\left(J_{\mathrm{K}}, \Lambda\right)$ for a global magnetic field (upper figure) and $\chi_{0, \text { loc }}^{\mathrm{ii}, \mathrm{NRG}}\left(J_{\mathrm{K}}, \Lambda\right)$ for a local magnetic field (lower figure) for the symmetric Kondo model with a constant density of states as a function $j=J_{\mathrm{K}} \rho_{0}(0)=J_{\mathrm{K}} / 2$ for various values of the Wilson parameter $\Lambda$. The lines represent the result of a second-order polynomial fit in $j$. To make the subleading terms discernible, we scale the susceptibilities by the universal part $\bar{\chi}_{0}(j)$ [see Eq. (122)]. The filled symbols at $j=0$ denote the analytical result (122). 
TABLE I. Coefficients of the subleading terms in the zero-field impurity-induced magnetic susceptibility for the ground state of the symmetric Kondo model with a constant density of states in Eq. (151) from NRG (see Fig. 12). The two values result from the two sequences of extrapolations (a) and (b) in $(\Lambda-1)$ and $j$ (see text). The analytic value for $s_{0}$ is given in Eq. (122). Also given are the coefficients for the fit in Eq. (153).

\begin{tabular}{lccccc}
\hline \hline & $s_{0}$ & $s_{1}$ & $s_{2}$ & $s_{0}$ & $\alpha$ \\
\hline$\hat{H}_{\mathrm{m}}$ & $0.1781^{(\mathrm{a})}$ & $-0.401^{(\mathrm{a})}$ & $0.348^{(\mathrm{a})}$ & $0.1784^{(\mathrm{a})}$ & $-2.33^{(\mathrm{a})}$ \\
& $0.1781^{(\mathrm{b})}$ & $-0.401^{(\mathrm{b})}$ & $0.348^{(\mathrm{b})}$ & $0.1784^{(\mathrm{b})}$ & $-2.35^{(\mathrm{b})}$ \\
$\hat{H}_{\mathrm{m}, \text { loc }}$ & $0.1734^{(\mathrm{a})}$ & $-0.269^{(\mathrm{a})}$ & $0.182^{(\mathrm{a})}$ & $0.1731^{(\mathrm{a})}$ & $-1.547^{(\mathrm{a})}$ \\
& $0.1734^{(\mathrm{b})}$ & $-0.269^{(\mathrm{b})}$ & $0.182^{(\mathrm{b})}$ & $0.1731^{(\mathrm{b})}$ & $-1.560^{(\mathrm{b})}$ \\
Exact & 0.171099 & & & 0.171099 & \\
\hline \hline
\end{tabular}

the Wilson parameter $\Lambda$ for a constant density of states, both for a global magnetic field and a local magnetic field at the impurity. The data for $\Lambda=1$ are the result of a quadratic fit in $(\Lambda-1)$ for given $J_{\mathrm{K}}$. We plot the ratio of the susceptibilities and the universal part $\bar{\chi}_{0}(j)$ [see Eq. (122)] to focus on the subleading terms. The NRG confirms the quadratic dependence of these terms on $j=\rho_{0}(0) J_{\mathrm{K}}$ for $J_{\mathrm{K}} \rightarrow 0$ :

$$
\frac{\chi_{0}^{\mathrm{ii}}\left(J_{\mathrm{K}}\right)}{\bar{\chi}_{0}(j)}=s_{0}+s_{1} j+s_{2} j^{2}+\cdots, \quad j=\rho_{0}(0) J_{\mathrm{K}} .
$$

We collect the results for $s_{0}, s_{1}, s_{2}$ in Table I.

We perform two sequences of extrapolations. In extrapolation (a), we start with a second-order polynomial fit in $(\Lambda-1)$ at fixed $J_{\mathrm{K}}$ and fit the resulting data in a secondorder polynomial fit in $j=J_{\mathrm{K}} \rho_{0}(0)$, as shown in Fig. 12. In extrapolation (b) we first extrapolate in $j$ to determine $s_{l}(\Lambda)$ and extrapolate these coefficients in $\Lambda$ afterward. NRG data for $0.15 \leqslant J_{\mathrm{K}} \leqslant 1.0$ and $1.8 \leqslant \Lambda \leqslant 3.2$ are included in the fit. As seen from the data in Table I, the results agree very well.

The parameter $s_{1}$ is related to Wilson's coefficients $\alpha_{1}$ and $c_{1}$ in Eq. (113) via

$$
s_{1}=-\frac{c_{1} / c_{0}+\alpha_{1}}{2 \mathcal{D} \sqrt{\pi e}}, \quad \frac{c_{1}}{c_{0}}=-2 \mathcal{D} \sqrt{\pi e} s_{1}-\alpha_{1},
$$

or $c_{1} \approx 0.45$, where we used $C=1$ for a constant density of states $\mathcal{D}=1, s_{0} \approx-0.40, \alpha_{1} \approx 1.5824$ [5], and $c_{0} \approx 0.6001$ from Eq. (116). Since $\left|c_{1} / c_{0}\right| \lesssim \alpha_{1}$, we could also have used

$$
\frac{\chi_{0}^{\mathrm{ii}}\left(J_{\mathrm{K}}\right)}{\bar{\chi}_{0}(j)} \approx s_{0} e^{-\alpha j}, \quad j=\rho_{0}(0) J_{\mathrm{K}}
$$

as our fit function. For completeness, the results for this fitting function are also included in Table I.

The constant term is identical in both cases $s_{0}^{\mathrm{NRG}} \approx s_{0, \text { loc }}^{\mathrm{NRG}} \approx$ $s_{0}=1 /(2 \sqrt{\pi e}) \approx 0.1709914$, where we used the analytic result from Eq. (122) for comparison. Since the NRG data for a global field show more scatter, the accuracy of $s_{0}$ is smaller than for a local field, the deviations are $4 \%$ for a global field and $1 \%$ for a local field. In any case, the accuracy is good enough to see that the prefactors of the first- and second-order terms are different for global and local magnetic fields $s_{1} \neq s_{1, \text { loc }}$ and $s_{2} \neq s_{2, \text { loc }}$. Different linear terms are also

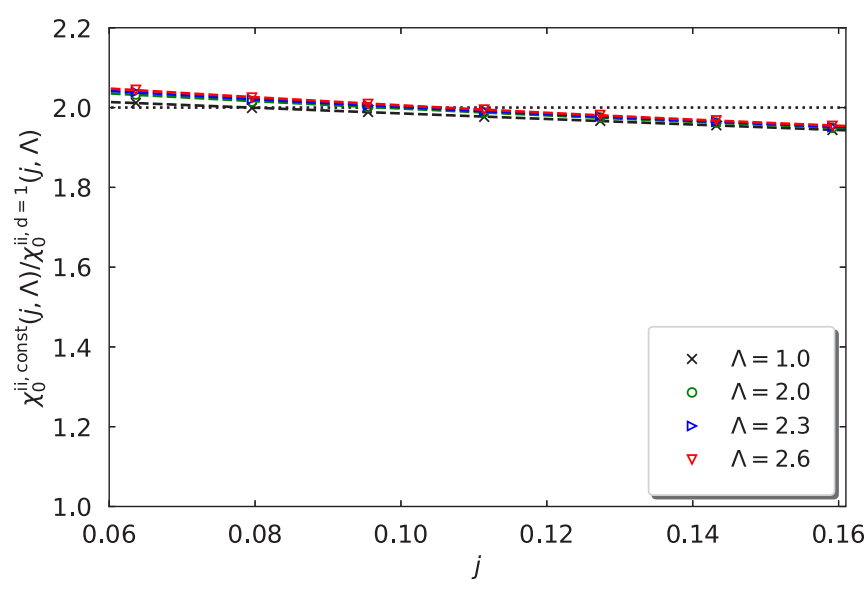

FIG. 13. Ratio between the zero-field impurity-induced magnetic susceptibility for a constant density of states $\chi_{0}^{\mathrm{ii}, \mathrm{NRG}, \mathrm{const}}\left(J_{\mathrm{K}}, \Lambda\right)$, and for a one-dimensional density of states $\chi_{0}^{\text {ii, NRG, }=1}\left(J_{\mathrm{K}}, \Lambda\right)$ for a global magnetic field as a function $j=J_{\mathrm{K}} \rho_{0}(0)$ for various values of the Wilson parameter $\Lambda$. The black crosses represent the result of a second-order polynomial fit in $\Lambda-1$. The line $C=2$ gives the result for $j=0$.

found in the Yosida wave function [compare Eqs. (72) and (73)].

\section{Impurity-induced magnetic susceptibility for a one-dimensional density of states}

In Fig. 13 we show the ratio between the zero-field impurity-induced magnetic susceptibility for a constant density of states $\chi_{0}^{\mathrm{ii}, \mathrm{NRG} \text {,const }}\left(J_{\mathrm{K}}, \Lambda\right)$ and for a one-dimensional density of states $\chi_{0}^{\mathrm{ii}, \mathrm{NRG}, \mathrm{d}=1}\left(J_{\mathrm{K}}, \Lambda\right)$ for a global magnetic field as a function $j=J_{\mathrm{K}} \rho_{0}(0)$ for various values of the Wilson parameter $\Lambda$. The extrapolated value for $\Lambda \rightarrow 1$ is very close to $C=2$ which is the exact result for $j=0$ [see Eq. (122)].

Apparently, the result holds for all $j \ll 1$, within the accuracy of the NRG calculations. This universality is also seen in the Yosida wave function [see Eq. (72)], where the subleading corrections are independent of the host-electron density of states. Therefore, we conjecture that the algebraic correction terms in Eq. (122) are universal in the sense that $s_{1} / s_{0} \approx-2.3$ and $s_{2} / s_{0} \approx 2$ do not depend on the shape of the host-electron density of states.

\section{Impurity spin susceptibility}

In Fig. 14 we show the zero-field impurity spin susceptibility as a function of $J_{\mathrm{K}}$ for various values of the Wilson parameter $\Lambda$ for a constant density of states, both for a global magnetic field and a local magnetic field at the impurity. The data for $\Lambda=1$ are the result of a quadratic fit in $(\Lambda-1)$ for given $J_{\mathrm{K}}$. Again, we plot the ratio of the susceptibilities and the universal part $\bar{\chi}_{0}(j)$ [see Eq. (122)] to focus on the subleading terms. The NRG confirms the quadratic dependence of these terms on $j=\rho_{0}(0) J_{\mathrm{K}}$ for $J_{\mathrm{K}} \rightarrow 0$ :

$$
\frac{\chi_{0}^{S}\left(J_{\mathrm{K}}\right)}{\bar{\chi}_{0}(j)}=S_{0}+S_{1} j+S_{2} j^{2}+\cdots, \quad j=\rho_{0}(0) J_{\mathrm{K}} .
$$



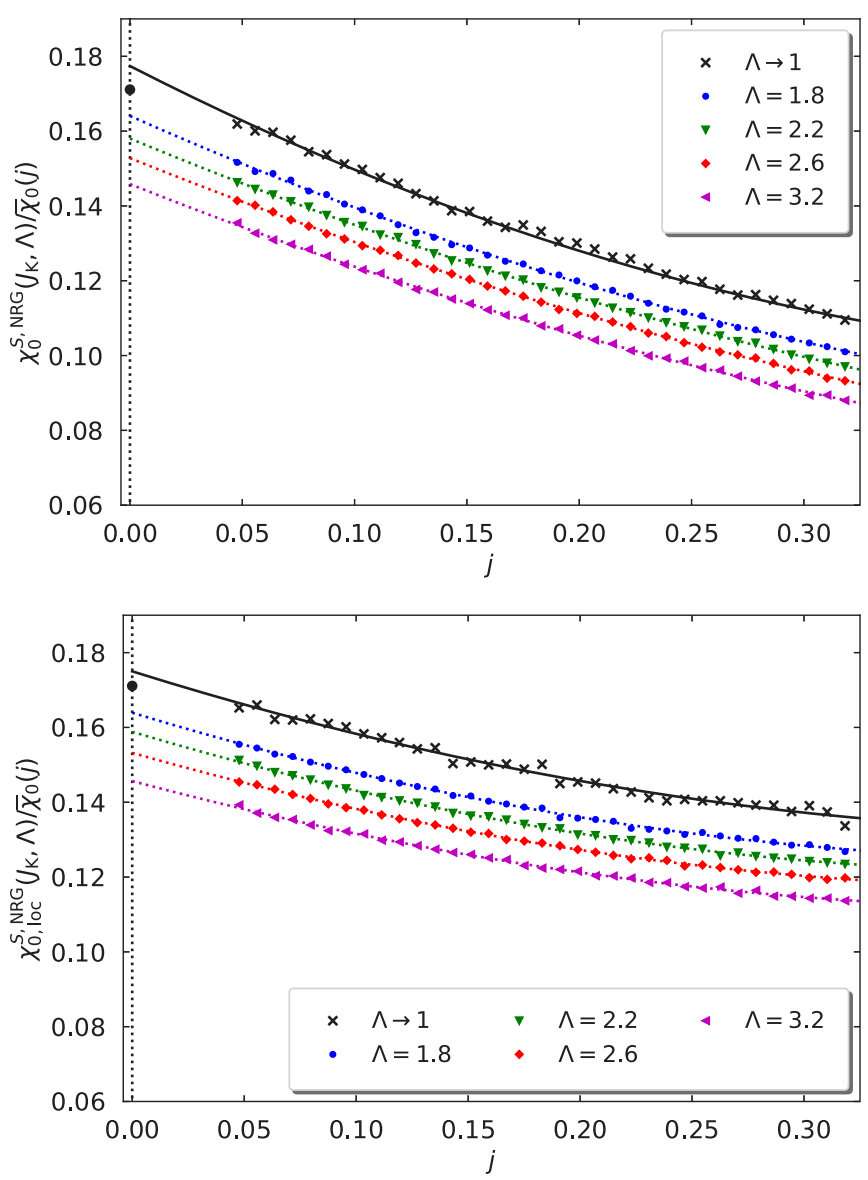

FIG. 14. Zero-field impurity spin susceptibility $\chi_{0}^{S, \mathrm{NRG}}\left(J_{\mathrm{K}}, \Lambda\right)$ for a global magnetic field (upper figure) and $\chi_{0, \mathrm{loc}}^{S, \mathrm{NRG}}\left(J_{\mathrm{K}}, \Lambda\right)$ for a local magnetic field (lower figure) for the symmetric Kondo model with a constant density of states as a function $j=J_{\mathrm{K}} \rho_{0}(0)=J_{\mathrm{K}} / 2$ for various values of the Wilson parameter $\Lambda$. The lines represent the result of a second-order polynomial fit in $j$. As in Fig. 12, we scale the susceptibilities by the universal part $\bar{\chi}_{0}(j)$. The filled symbols at $j=0$ denote the analytical result (122).

We collect the results for $S_{0}, S_{1}, S_{2}$ in Table II. Note that we use capital letters here to distinguish the coefficients $s_{l}$ for the impurity-induced susceptibility from the coefficients $S_{l}$ for the impurity spin susceptibility. We also include the results from

TABLE II. Coefficients of the subleading terms in the zero-field impurity spin susceptibility for the ground state of the symmetric Kondo model with a constant density of states in Eq. (151) from NRG (see Fig. 14). The two values result from the two sequences of extrapolations (a) and (b) in $(\Lambda-1)$ and $j$ (see text). The analytic value for $S_{0}=s_{0}$ is given in Eq. (122). Also given are the coefficients for the fit in Eq. (153).

\begin{tabular}{lccccc}
\hline \hline & $S_{0}$ & $S_{1}$ & $S_{2}$ & $S_{0}$ & $\alpha^{S}$ \\
\hline$\hat{H}_{\mathrm{m}}$ & $0.1774^{(\mathrm{a})}$ & $-0.307^{(\mathrm{a})}$ & $0.300^{(\mathrm{a})}$ & $0.1738^{(\mathrm{a})}$ & $-1.49^{(\mathrm{a})}$ \\
& $0.1774^{(\mathrm{b})}$ & $-0.307^{(\mathrm{b})}$ & $0.300^{(\mathrm{b})}$ & $0.1738^{(\mathrm{b})}$ & $-1.50^{(\mathrm{b})}$ \\
$\hat{H}_{\mathrm{m}, \text { loc }}$ & $0.1753^{(\mathrm{a})}$ & $-0.193^{(\mathrm{a})}$ & $0.218^{(\mathrm{a})}$ & $0.1707^{(\mathrm{a})}$ & $-0.766^{(\mathrm{a})}$ \\
& $0.1753^{(\mathrm{b})}$ & $-0.193^{(\mathrm{b})}$ & $0.218^{(\mathrm{b})}$ & $0.1707^{(\mathrm{b})}$ & $-0.766^{(\mathrm{b})}$ \\
Exact & 0.171099 & & & 0.171099 & \\
\hline
\end{tabular}

the extrapolation analogous to Eq. (153) which provides the coefficient $\alpha^{S}$ from an exponential extrapolation. NRG data for $0.15 \leqslant J_{\mathrm{K}} \leqslant 1.0$ and $1.8 \leqslant \Lambda \leqslant 3.2$ are included in the fit.

For the impurity spin susceptibility we also find the expected result $S_{0}=s_{0}=1 /(2 \sqrt{\pi e}) \approx 0.1709914$, irrespective of a global or a local field, with deviations of about $4 \%$. Again, the first- and second-order coefficients $S_{1}$ and $S_{2}$ depend on whether the magnetic field is applied globally or locally.

To assess the accuracy of our extrapolations, we compare the results for $\chi_{0}^{S}\left(J_{\mathrm{K}}\right)$ and $\chi_{0, \text { loc }}^{\mathrm{ii}}\left(J_{\mathrm{K}}\right)$ which should be equal [see Eq. (24)]. We see that $s_{1, \text { loc }}=-0.269$ agrees reasonably well with $S_{1}=-0.307$, with a deviation of the order of $10 \%$. However, $s_{2, \text { loc }}=0.182$ and $S_{2}=0.300$ are off by more than $40 \%$. Since the data for the local susceptibilities are better than those for the global susceptibilities, we argue that the data for $s_{1, \text { loc }}$ and $s_{2, \text { loc }}$ are more reliable. Nevertheless, the comparison indicates that the values for $s_{1}$ and $S_{1}\left(s_{2}\right.$ and $\left.S_{2}\right)$ have an uncertainty of several (ten) percent.

\section{Magnetic susceptibilities for strong coupling 1. Impurity-induced susceptibility}

In Fig. 15 we show the zero-field impurity-induced susceptibility with a global and a local field for $J_{\mathrm{K}} \geqslant 1$ from NRG in comparison with the Gutzwiller result. In general, we calculate the impurity-induced magnetization for small local fields and determine the susceptibility from the slope. In the strong-coupling region, this procedure becomes unstable in NRG so that we determine the susceptibility from the second derivative of the ground-state energy with respect to the global field [see Eq. (21)].

As seen from Fig. 15, the Gutzwiller wave function almost perfectly reproduces the NRG data for $J_{K} \gtrsim 1.5$. For intermediate to strong couplings, the Gutzwiller wave function is an excellent trial state for the Kondo model.

The strong-coupling asymptotics is shown in Fig. 2. The asymptotic formulas (103) and (104) are applicable for $J_{\mathrm{K}} \gtrsim 4$.

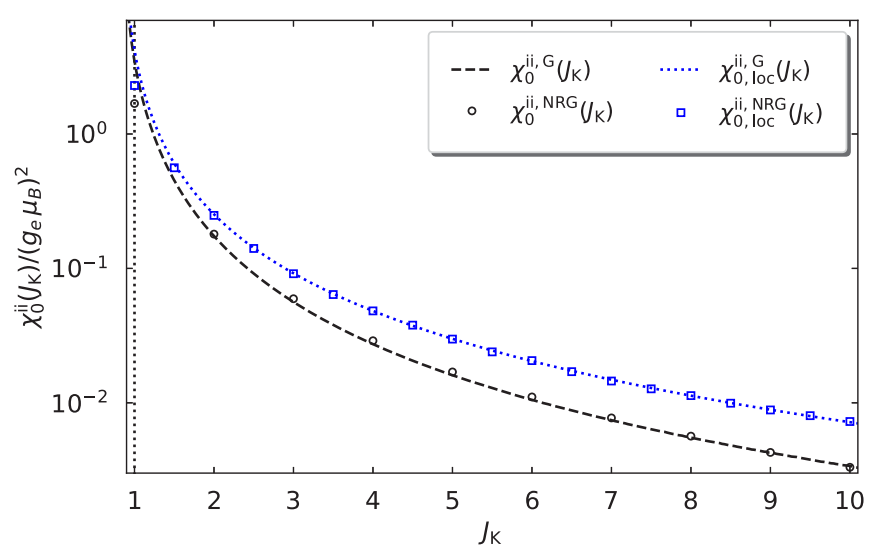

FIG. 15. Zero-field impurity-induced susceptibilities $\chi_{0}^{\mathrm{ii}}\left(J_{\mathrm{K}}\right)$ and $\chi_{0, \text { loc }}^{\mathrm{ii}}\left(J_{\mathrm{K}}\right)$ on a logarithmic scale for a global and a local magnetic field for the one-dimensional symmetric Kondo model as a function of $J_{\mathrm{K}}$ for $J_{\mathrm{K}} \geqslant 1$ from NRG and the Gutzwiller wave function. 


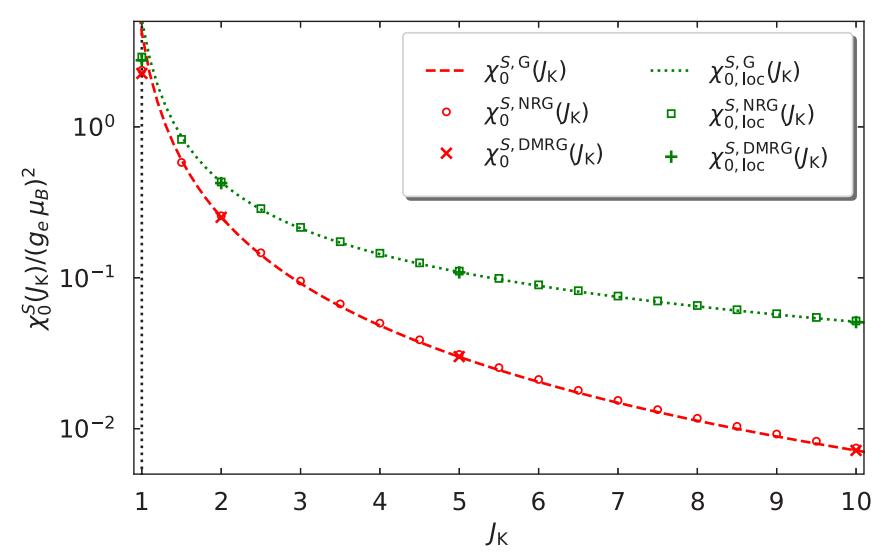

FIG. 16. Zero-field impurity spin susceptibilities $\chi_{0}^{S}\left(J_{\mathrm{K}}\right)$ and $\chi_{0, \text { loc }}^{S}\left(J_{\mathrm{K}}\right)$ on a logarithmic scale for a global and a local magnetic field for the one-dimensional symmetric Kondo model as a function of $J_{\mathrm{K}}$ for $J_{\mathrm{K}} \geqslant 1$ from NRG, DMRG, and the Gutzwiller wave function.

\section{Impurity spin susceptibility}

In Fig. 16 we show the zero-field impurity spin susceptibility for $J_{\mathrm{K}} \geqslant 1$. For intermediate to strong couplings, we find an excellent agreement between the data from NRG and DMRG both in the presence of global and local magnetic fields. Again, the Gutzwiller wave function provides an excellent analytic estimate for the zero-field susceptibilities for all $J_{\mathrm{K}} \gtrsim 1.5$.

The strong-coupling asymptotics is shown in Fig. 2. The limiting expressions (101) and (102) become applicable for $J_{\mathrm{K}} \gtrsim 4$.

\section{Impurity-induced magnetization and impurity spin polarization for weak coupling}

Next, we address the impurity-induced magnetization and the impurity spin polarization as a function of the external field for weak coupling. The comparison of Bethe-ansatz results and NRG data was done only recently [13].

\section{Impurity-induced magnetization}

In Fig. 17 we show the impurity-induced magnetization $m^{\mathrm{ii}}(B) /\left(g_{e} \mu_{\mathrm{B}}\right)$ for $J_{\mathrm{K}}=0.5$ as a function of the global field $B \leqslant 0.014$ as obtained from the Yosida wave function, in comparison with NRG data and results from the Bethe ansatz. We omit the Gutzwiller results because Gutzwiller theory predicts a finite magnetization even at $B=0^{+}$and thus fails to reproduce the paramagnetic phase at $J_{\mathrm{K}}=0.5$. As discussed in Sec. VIII A, DMRG cannot faithfully reproduce the magnetization for $J_{\mathrm{K}}=0.5$ because the tractable system sizes are too small. Therefore, we do not show DMRG data for the impurity-induced magnetization in Fig. 17.

At $J_{\mathrm{K}}=0.5$ and in one dimension where $\rho_{0}(0)=1 / \pi$, we have $j=\rho_{0}(0) J_{\mathrm{K}}=1 /(2 \pi) \approx 0.159$. The zero-field susceptibility is quite large already. In units of $\left(g_{e} \mu_{\mathrm{B}}\right)^{2}$ we have $\chi_{0}^{\mathrm{ii}}[j=1 /(2 \pi)] \approx \bar{\chi}_{0}[1 /(2 \pi)] s_{0}\left[1+\left(s_{1} / s_{0}\right)(2 \pi)+\right.$ $\left(s_{2} / s_{0}\right)(2 \pi)^{2}$ ] $\approx 79$ [see Eq. (122)], where we employ $s_{0}=$ $1 /(2 \mathcal{D} C \sqrt{\pi e}) \approx 0.0856$ for $C^{d=1}=2$ and $\mathcal{D}=1$, and make the assumption that the ratios $s_{1} / s_{0} \approx-2.3$ and $s_{2} / s_{0} \approx 2$

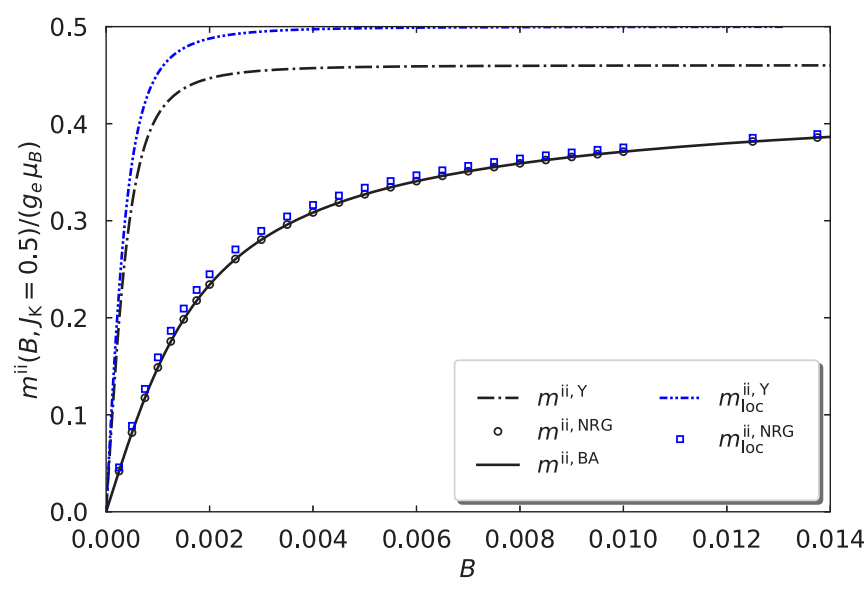

FIG. 17. Impurity-induced magnetization $m^{\mathrm{ii}}(B) /\left(g_{e} \mu_{\mathrm{B}}\right)$ for $J_{\mathrm{K}}=0.5$ for the one-dimensional symmetric Kondo model as a function of global/local magnetic fields $B$ from the Yosida wave function, NRG, and the Bethe ansatz.

do not depend on the host-electron density of states, and are thus obtained from the values in Table I. A large zero-field susceptibility implies a sharp increase of the magnetization for small fields as seen in Fig. 17. The Yosida state overestimates the zero-field susceptibility by more than a factor of $5, \chi_{0}^{\mathrm{ii}, \mathrm{Y}}[j=1 /(2 \pi)] \approx 433$ [see Eq. (72)]. Thus, the Yosida wave function also overestimates the magnetization for small and intermediate fields (see Fig. 17).

In the Bethe ansatz, the sharp increase at small fields is followed by a very slow convergence to the limiting value $m^{\mathrm{ii}}\left(B \gg T_{\mathrm{H}}\right)=\frac{1}{2}$. The resulting broad magnetization plateau originates from the logarithmic terms in the Bethe-ansatz solution [see Eq. (VI-86) in the Supplemental Material [23]]. The NRG results lie on top of the Bethe-ansatz data which show that the Bethe-ansatz expressions (128) and (129) remain valid up to $J_{\mathrm{K}}$ of the order of a quarter of the bandwidth, as long as $T_{1}$ is determined from the exact zero-field susceptibility from Eq. (130).

In Fig. 17 we also show the impurity-induced magnetization $m_{\mathrm{loc}}^{\mathrm{ii}}(B)$ as a function of a local magnetic field for $J_{\mathrm{K}}=0.5$. For small values of the Kondo coupling, the differences between globally and locally applied external fields are fairly small. The impurity-induced magnetization in the presence of a local field is a few percent larger than in the presence of a global field. This can be deduced from Eq. (151) which shows that the ratio of the zero-field susceptibilities is close to unity, $\left[1+\left(s_{1, \mathrm{loc}} / s_{0}\right) j+\left(s_{2, \mathrm{loc}} / s_{0}\right) j^{2}\right] /[1+$ $\left.\left(s_{1} / s_{0}\right) j+\left(s_{2} / s_{0}\right) j^{2}\right] \approx 1.13$ at $j=1 /(2 \pi)$ where the data are taken from Table I. Likewise, the differences between the impurity-induced magnetization $m^{\mathrm{ii}}(B)$ and the impurity spin polarization $m^{S}(B)$ are small at small $J_{\mathrm{K}},\left[1+\left(S_{1} / s_{0}\right) j+\right.$ $\left.\left(S_{2} / s_{0}\right) j^{2}\right] /\left[1+\left(s_{1} / s_{0}\right) j+\left(s_{2} / s_{0}\right) j^{2}\right] \approx 1.12$ at $j=1 /(2 \pi)$, where the data are taken from Tables I and II. This has been noted previously in Ref. [13].

In the Yosida wave function, the differences between the impurity-induced magnetization for global and local fields are more pronounced. In the case of a local field, the impurityinduced magnetization in the Yosida wave function quickly reaches the maximal value of one-half. For a global field, the 

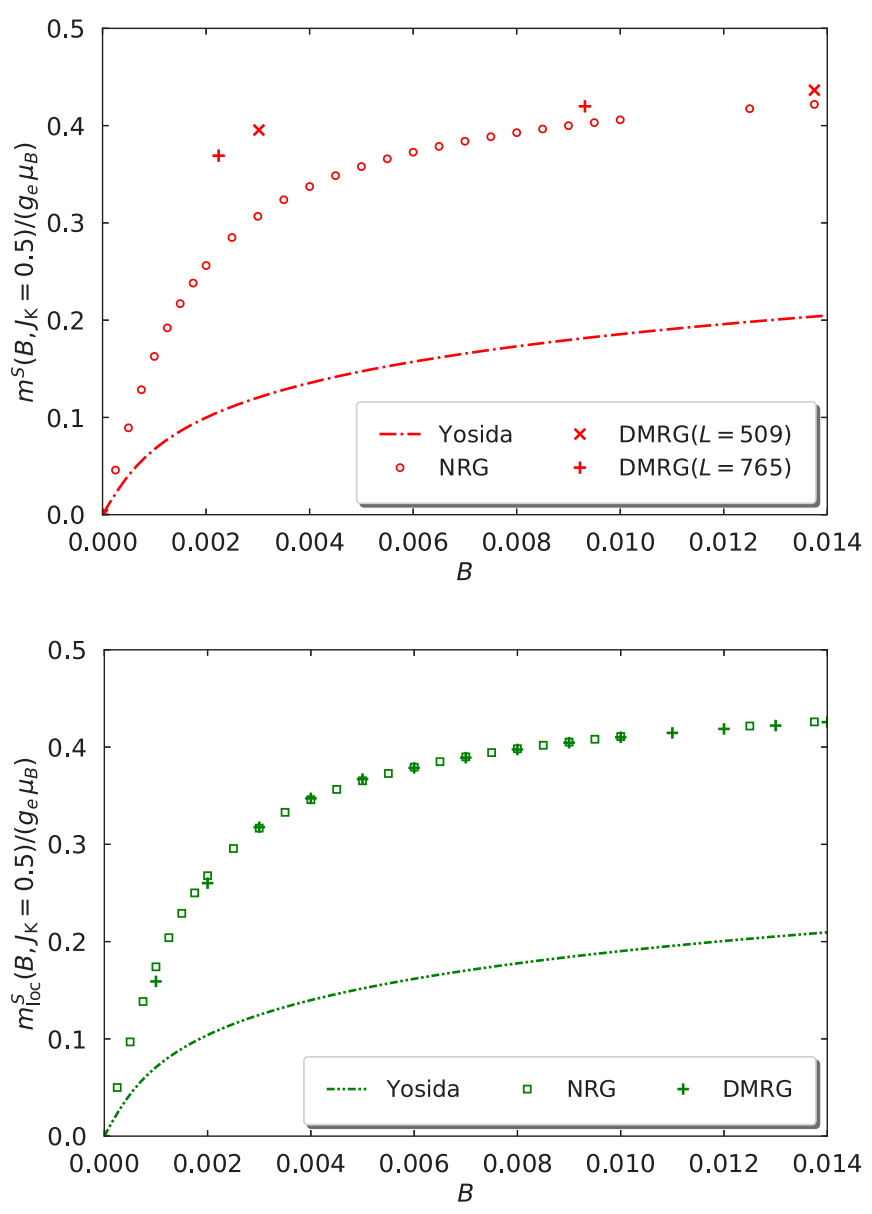

FIG. 18. Impurity spin polarization $S^{z}(B)=m^{S}(B) /\left(g_{e} \mu_{\mathrm{B}}\right)$ as a function of a global magnetic field (upper figure) and $S_{\mathrm{loc}}^{z}(B)=$ $m_{\mathrm{loc}}^{S}(B) /\left(g_{e} \mu_{\mathrm{B}}\right)$ as a function of a local magnetic field (lower figure) for the one-dimensional symmetric Kondo model at $J_{\mathrm{K}}=0.5$ from NRG, DMRG, and the Yosida wave function.

impurity-induced magnetization saturates below this value, in contrast to the exact solution. Altogether, the Yosida wave function correctly describes some gross aspects of the magnetization curves (large zero-field susceptibility, monotonous increase to saturation) but it fails to reproduce them in detail, e.g., the small difference between global and local fields.

\section{Impurity spin polarization}

In Fig. 18 we show the impurity spin polarization in the presence of a global and a local field, respectively. As seen from the previous Sec. IXD1, the NRG is the best method to study magnetic properties of the Kondo model at weak coupling. Therefore, its results can be used to assess the quality of all other methods.

In Fig. 18 we leave out the Gutzwiller results because the spin polarization is finite at $J_{\mathrm{K}}=0.5$, and almost independent of $B$ for all $0 \leqslant B<0.014$, in contrast to the NRG data. The Yosida wave function provides qualitatively correct results but grossly underestimates the spin polarization in both cases. Therefore, the Yosida wave function does not provide an acceptable description of the impurity spin polarization.

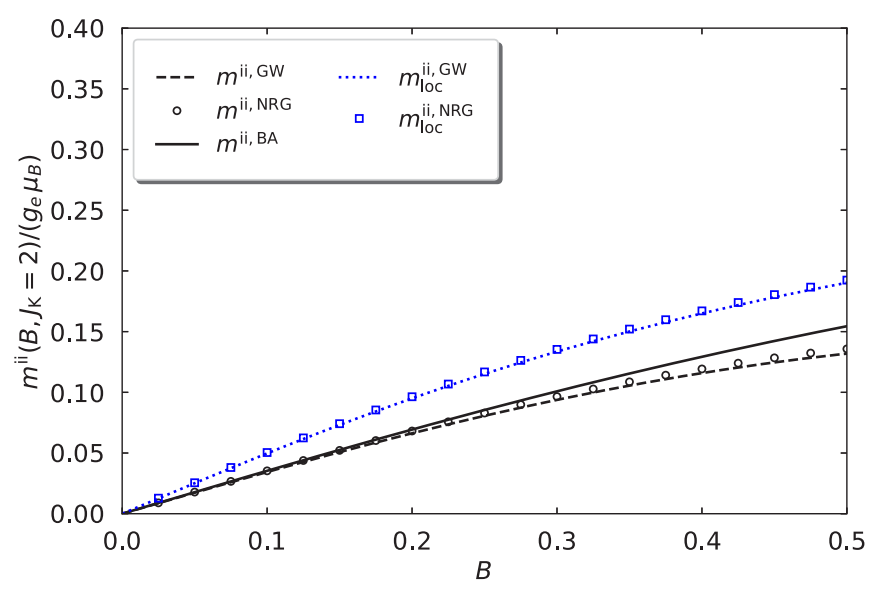

FIG. 19. Impurity-induced magnetization $m^{\mathrm{ii}}(B) /\left(g_{e} \mu_{\mathrm{B}}\right)$ for $J_{\mathrm{K}}=2$ for the one-dimensional symmetric Kondo model as a function of a global/local magnetic field $B$ from Bethe ansatz, NRG, and the Gutzwiller wave function.

As discussed in Sec. VIII A, DMRG requires very large system sizes for small Kondo couplings to calculate the impurity spin polarization in the presence of a small global field. Therefore, at $J_{\mathrm{K}}=0.5$ the DMRG and NRG data agree only for $B \gtrsim 0.01$. For smaller $B$ values, DMRG substantially overestimates the magnetization, displaying large finite-size effects. Recall that DMRG works for fixed total spin $S_{\text {tot }}^{z}$ so that only specific values for $B$ are accessible. This limitation does not apply for a purely local field. It can be tuned freely also in DMRG so that a finite-size extrapolation of the DMRG data for the impurity spin polarization is unproblematic. As seen from Fig. 18, the NRG and DMRG data perfectly agree for the impurity spin polarization in the presence of a local magnetic field. Alternatively, since $m_{\mathrm{loc}}^{S}$ is a thermodynamic quantity, it can also be obtained from the derivative of the excess ground-state energy with respect to the external field [see Eq. (22)]. Both approaches lead to the same results.

\section{E. Impurity-induced magnetization and impurity spin polarization for strong coupling}

Lastly, we address the impurity-induced magnetization and the impurity spin polarization as a function of the external field for strong coupling.

\section{Impurity-induced magnetization}

In Fig. 19 we show the impurity-induced magnetization as a function of $B$ at $J_{\mathrm{K}}=2$. When the Kondo coupling reaches the bandwidth, the singlet state between the impurity spin and the band electron at the origin is tightly bound so that the susceptibility is small and even a sizable field can barely polarize the singlet. Therefore, the impurity magnetization remains small for $B \leqslant 0.5$.

The NRG data lie on top of the analytic results from the Gutzwiller wave function. This again shows that the Gutzwiller wave function is an excellent trial state for the Kondo model at large couplings. At $J_{\mathrm{K}}=2$, the Bethe ansatz is no longer applicable, and sizable differences between NRG/Gutzwiller results and Bethe-ansatz predictions become 


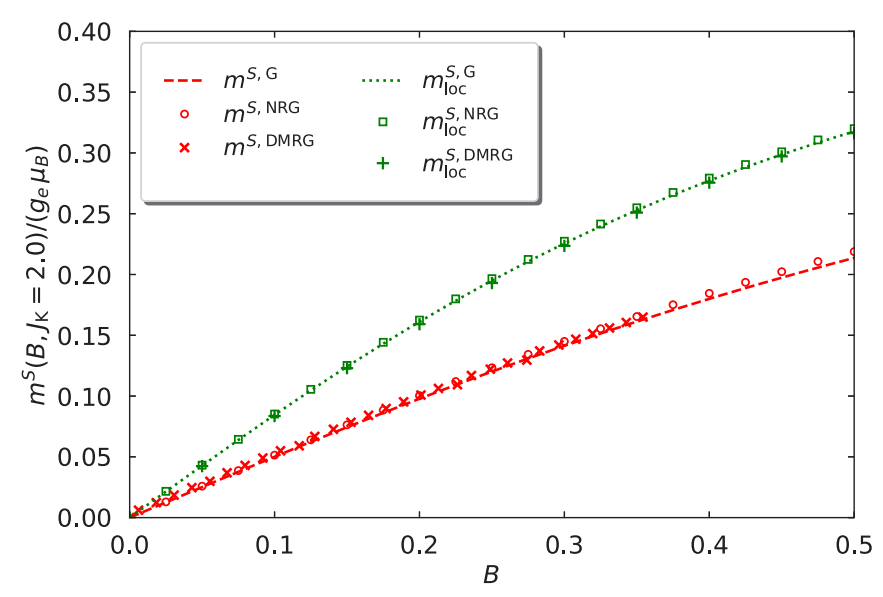

FIG. 20. Impurity spin polarization $S^{z}(B)=m^{S}(B) /\left(g_{e} \mu_{\mathrm{B}}\right)$ for $J_{\mathrm{K}}=2$ for the one-dimensional symmetric Kondo model as a function of a global/local magnetic field $B$ from NRG, DMRG, and the Gutzwiller wave function.

discernible at large $B$, despite the fact that the linear term is fixed to the exact susceptibility. Values $J_{\mathrm{K}} \gtrsim W$ are beyond the Bethe-ansatz description.

\section{Impurity spin polarization}

Finally, in Fig. 20 we show the impurity spin polarization as a function of $B$ at $J_{\mathrm{K}}=2$. For large Kondo couplings, the finite-size restrictions imposed on DMRG discussed in Sec. VIII A are far less severe, and the results for $L=763$ sites for a global field perfectly reproduce the NRG data for $0 \leqslant B<0.5$. The same perfect agreement between DMRG and NRG data is seen for the case of a local field whose value can be chosen freely also for the DMRG calculations.

The analytic results from the Gutzwiller wave function lie on top the the NRG/DMRG data for both a global and a local field. Again, the Gutzwiller wave function is seen to provide an excellent trial state for the Kondo model at large couplings.

\section{CONCLUSIONS}

In this last section, we summarize our central findings and discuss our main results.

\section{A. Summary}

In our work, we investigated the symmetric single-impurity Kondo model on a chain at zero temperature. As a function of the Kondo coupling $J_{\mathrm{K}}$, we studied the ground-state energy, the local spin correlation function, the impurity spin polarization and impurity-induced magnetization, and the corresponding zero-field magnetic susceptibilities for global and local external fields $B$. Some of these quantities, e.g., the ground-state energy and the local spin correlation function, are related by the Hellmann-Feynman theorem that also holds for variational wave functions (see Appendix B 1).

We calculated the ground-state energy and the local spin correlation function in weak-coupling and strong-coupling perturbation theory at $B=0$ as benchmark for our approaches. Since we are not aware of these results in the literature, we include the calculations as Appendix B 2 for weak coupling and as Appendix B 3 for strong coupling.

As the first of three analytical variational methods, we analyzed the first-order Lanczos state at $B=0$, as done by Mancini and Mattis [12] for a constant density of states. We recapitulated the Lanczos method and performed the calculation of the first-order coefficients in Appendix B 2. Since the analytic calculations for the Lanczos scheme become cumbersome beyond first order and the first-order results are not impressive, we refrain from further pursuing this approach.

Second, we extended and evaluated the Yosida wave function $[16,17]$ to include external magnetic fields. The Yosida wave function provides a simple description of the Kondosinglet ground state with an exponentially small binding energy at small Kondo couplings. As has been anticipated in the literature but was never worked out explicitly before, we show that the Yosida binding energy translates into an exponentially large zero-field magnetic susceptibility at weak coupling.

Third, in this work we introduced and employed the Gutzwiller variational state [18,19] at finite fields. The latter provides a Hartree-Fock-type description of the Kondo model that guarantees that the impurity is singly occupied. As a central result of our work, we showed that the Gutzwiller wave function becomes exact in the limit of large Kondo couplings $J_{\mathrm{K}} \gg W$. The evaluation of the Gutzwiller wave function required the solution of the noninteracting singleimpurity Anderson model (SIAM) in the presence of potential scattering. For completeness, this was done in Appendix B 4.

As numerical techniques, we employed the numerical renormalization group (NRG) [5-8] and the density-matrix renormalization group (DMRG) [20-22] methods. Using the DMRG method we addressed finite half-chains of length $L / 2$, and performed quadratic fits in $1 / L$ for physical quantities to extrapolate to the thermodynamic limit $L \rightarrow \infty$. To the best of our knowledge, the DMRG was not extensively applied to the single-impurity Kondo model before. In DMRG, the system sizes are limited to $L \lesssim 10^{3}$ so that we could not address the impurity-induced magnetizations and the zerofield susceptibilities at small Kondo couplings.

The NRG permits the accurate calculation of all groundstate quantities as a function of the Wilson parameter $\Lambda$. We performed quadratic fits in $(\Lambda-1)$ to extrapolate our data to the limit $\Lambda=1$. We employed the correction factor derived by Krishna-murthy, Wilkins, and Wilson [6,7] because it improved the quality of the extrapolations. Such extrapolations are often omitted in the literature. However, our comparison with DMRG and Bethe-ansatz data shows that the extrapolation in $\Lambda$ considerably improves the agreement.

Since the Bethe ansatz solves a Kondo model with linear dispersion and infinite bandwidth, a direct comparison of physical quantities is not easy because there is a nontrivial relation between the Kondo couplings $J_{\mathrm{K}}^{\mathrm{BA}}$ and $J_{\mathrm{K}}$ used in the Bethe ansatz and in the lattice model, respectively. As a by-product of our study, we showed in Appendix B 5 that the ground-state energy in the Bethe ansatz is zero, up to corrections of the order $\left(J_{\mathrm{K}}^{\mathrm{BA}}\right)^{3}$. Thus, the ground-state energy from Bethe ansatz cannot be used for a comparison with the lattice model. 
Using known results from Andrei, Furuya, and Lowenstein [11], rederived in Appendix B 6 and extended to a regular host-electron density of states, we expressed $J_{\mathrm{K}}^{\mathrm{BA}}$ as a series expansion in $J_{\mathrm{K}}$, and gave analytic expressions for the leadingorder terms in Eq. (123). While the logarithmic term is known from Wilson's RG, in this work we analytically determined also the constant term for a regular density of states.

When the zero-field susceptibility from NRG is used, the impurity-induced magnetization from Bethe ansatz and from NRG were seen to agree perfectly. This was observed earlier in the NRG analysis of the impurity magnetization by Schnack and Höck [13]. In our extensive analysis, we showed that the various zero-field susceptibilities have a universal smallcoupling limit [see Eq. (122)]. For finite $J_{K}$, however, the impurity spin polarization and the impurity-induced magnetization at global and local fields differ from each other by a factor that goes to unity for $J_{K} \rightarrow 0$. Using NRG, in this work we calculated the corrections numerically, with an accuracy of some $10 \%$. Since two of the zero-field susceptibilities agree [see Eq. (24)], we found a reliable method to assess the accuracy of the NRG calculations for the zero-field susceptibilities that is independent of an exact solution.

\section{B. Discussion}

The ground state of the symmetric single-impurity Kondo model describes a Kondo singlet formed by the impurity spin and its host-electron screening cloud. Unfortunately, it is by no means easy to formulate a concise, analytically tractable variational wave function that adequately describes the ground state for all couplings.

In this work we showed that the Gutzwiller wave function provides an excellent trial state for large Kondo couplings $J_{\mathrm{K}} \gtrsim W$, where $W=2$ is the bandwidth. It reproduces the ground-state energy, the local spin correlation, the impurity spin polarization and impurity-induced magnetization, and the corresponding zero-field susceptibilities from NRG and DMRG with high accuracy. Unfortunately, it displays a Hartree-Fock-type transition to a state with an oriented impurity spin below $J_{\mathrm{K}, \mathrm{c}}^{\mathrm{G}} \approx 0.893$ that is not contained in the exact solution of the model. Therefore, the Gutzwiller wave function does not describe the small-coupling limit properly.

For weak coupling, the Yosida wave function reproduces the exponential divergence of the zero-field susceptibilities known from Bethe ansatz and NRG but it fails to provide a good variational bound on the ground-state energy for all couplings. Eventually, it becomes unstable for large Kondo couplings. Thus, the Yosida and Gutzwiller variational approaches provide a complementary view on the Kondo-singlet ground state.

The DMRG method numerically determines an optimal variational ground state for the Kondo model on finite lattices. Although not specifically designed for impurity models, the method works very well as long as all energy scales lie within the DMRG energy resolution $\Delta \epsilon=W / L$. However, the Kondo temperature in the Kondo model becomes exponentially small for $J_{\mathrm{K}} \ll W$ so that the calculation of magnetic properties using DMRG is limited to $J_{\mathrm{K}} \gtrsim 0.6$ for the onedimensional density of states.
Other quantities such as the ground-state energy and the local spin correlation function are unproblematic. For $J_{\mathrm{K}}=2$, the results from NRG and DMRG agree perfectly, not only for the ground-state energy and local spin correlation, but also for the impurity spin polarization and zero-field susceptibility. The DMRG can also be applied to impurity problems as long as all intrinsic energy scales can be resolved appropriately.

The NRG was specifically designed to treat exponentially small energy scales in impurity models and thus works exceedingly well for the Kondo model. In this work we showed that NRG also permits the accurate calculation of the groundstate energy and local spin correlation function. In particular, we found that an extrapolation $\Lambda \rightarrow 1$ is required whereby the correction factor derived by Krishna-murthy, Wilkins, and Wilson [6,7] is helpful to improve the extrapolations.

In contrast to the single-impurity Anderson model [28], the Bethe-ansatz results for the Kondo model cannot be directly compared to numerical results because the continuum and lattice models differ in their coupling constants. Therefore, at zero temperature, only the impurity-induced magnetization for small Kondo couplings can eventually be compared to the $\mathrm{NRG}$ data. For this reason, it was indispensable to generate accurate data from NRG for comparison with the analytical and numerical variational methods (Lanczos, Yosida, Gutzwiller, DMRG) employed in this work.

\section{ACKNOWLEDGMENTS}

This research has been supported in part by the Hungarian National Research, Development and Innovation Office (NKFIH) through Grants No. K120569 and No. PD-17-125261 and the Hungarian Quantum Technology National Excellence Program (Project No. 2017-1.2.1-NKP-2017-00001). Support by the Alexander von Humboldt foundation (Ö.L.) and by the International Collaborative Research Center TRR 160 Coherent manipulation of interacting spin excitations in tailored semiconductors (F.B.A., F.E.) is gratefully acknowledged.

\section{APPENDIX A: TECHNICAL DETAILS}

\section{Diagonalization of the kinetic energy operator}

We use open boundary conditions and define the operators for standing waves

$$
\hat{a}_{k, \sigma}=\sqrt{\frac{2}{L+1}} \sum_{n=-(L-1) / 2}^{(L-1) / 2} \sin \left(\frac{\pi k n}{L+1}+\frac{\pi k}{2}\right) \hat{c}_{n, \sigma} .
$$

We may formally include the operators $\hat{c}_{ \pm(L+1) / 2, \sigma}$ at sites $n= \pm(L+1) / 2$ because they do not enter the standing-wave operators $\hat{a}_{k, \sigma}$. The inverse transformation reads as

$$
\hat{c}_{n, \sigma}=\sqrt{\frac{2}{L+1}} \sum_{k=1}^{L} \sin \left[\frac{\pi k}{L+1}\left(n+\frac{L+1}{2}\right)\right] \hat{a}_{k, \sigma} .
$$

The kinetic energy becomes diagonal

$$
\hat{T}=\sum_{k=1, \sigma}^{L} \epsilon_{k} \hat{a}_{k, \sigma}^{+} \hat{a}_{k, \sigma}
$$


with the dispersion relation $(1 \leqslant k \leqslant L)$

$$
\epsilon_{k}=-2 t \cos \left(\frac{\pi k}{L+1}\right)
$$

\section{Half-chain geometry}

Equation (A2) shows that

$$
\hat{c}_{0, \sigma}=\sqrt{\frac{2}{L+1}} \sum_{k=1}^{L} \sin \left[\frac{\pi k}{2}\right] \hat{a}_{k, \sigma} .
$$

Therefore, the standing waves with even $k$ do not couple to the chain center, and drop out of the problem.

\section{a. Canonical transformation to parity eigenstates}

The reason for this decoupling is parity symmetry. To make it transparent in the real-space representation, we introduce the operators for $n=1,2, \ldots,(L-1) / 2$

$$
\begin{aligned}
& \hat{C}_{n, \sigma}=\sqrt{\frac{1}{2}}\left(\hat{c}_{n, \sigma}+\hat{c}_{-n, \sigma}\right), \\
& \hat{S}_{n, \sigma}=\sqrt{\frac{1}{2}}\left(\hat{c}_{n, \sigma}-\hat{c}_{-n, \sigma}\right),
\end{aligned}
$$

with the inverse transformation

$$
\begin{gathered}
\hat{c}_{n, \sigma}=\sqrt{\frac{1}{2}}\left(\hat{C}_{n, \sigma}+\hat{S}_{n, \sigma}\right), \\
\hat{c}_{-n, \sigma}=\sqrt{\frac{1}{2}}\left(\hat{C}_{n, \sigma}-\hat{S}_{n, \sigma}\right) .
\end{gathered}
$$

Moreover, we set $\hat{C}_{0, \sigma} \equiv \hat{c}_{0, \sigma}$ for notational consistency. The transformation is a canonical basis transformation.

Then, the kinetic energy becomes

$$
\hat{T}=\hat{T}^{C}+\hat{T}^{S},
$$

where the two commuting parts of the kinetic energy are given by

$$
\begin{aligned}
\hat{T}^{C}= & -\sqrt{2} t \sum_{\sigma}\left(\hat{C}_{0, \sigma}^{+} \hat{C}_{1, \sigma}+\hat{C}_{1, \sigma}^{+} \hat{C}_{0, \sigma}\right) \\
& +(-t) \sum_{n=1, \sigma}^{(L-3) / 2}\left(\hat{C}_{n+1, \sigma}^{+} \hat{C}_{n, \sigma}+\hat{C}_{n, \sigma}^{+} \hat{C}_{n+1, \sigma}\right), \\
\hat{T}^{S}= & (-t) \sum_{n=1, \sigma}^{(L-3) / 2}\left(\hat{S}_{n+1, \sigma}^{+} \hat{S}_{n, \sigma}+\hat{S}_{n, \sigma}^{+} \hat{S}_{n+1, \sigma}\right) .
\end{aligned}
$$

Therefore, the $S$ electrons drop out of the problem, and the Kondo Hamiltonian reduces to

$$
\hat{H}_{\mathrm{K}}^{C}=\hat{T}^{C}+\hat{V}_{\mathrm{sd}}+\hat{H}_{\mathrm{m}}
$$

with

$$
\begin{aligned}
\hat{V}_{\mathrm{sd}}= & \frac{J_{\mathrm{K}}}{2}\left(\hat{C}_{0, \uparrow}^{+} \hat{C}_{0, \downarrow} \hat{d}_{\downarrow}^{+} \hat{d}_{\uparrow}+\hat{C}_{0, \downarrow}^{+} \hat{C}_{0, \uparrow} \hat{d}_{\uparrow}^{+} \hat{d}_{\downarrow}\right) \\
& +\frac{J_{\mathrm{K}}}{4}\left(\hat{d}_{\uparrow}^{+} \hat{d}_{\uparrow}-\hat{d}_{\downarrow}^{+} \hat{d}_{\downarrow}\right)\left(\hat{C}_{0, \uparrow}^{+} \hat{C}_{0, \uparrow}-\hat{C}_{0, \downarrow}^{+} \hat{C}_{0, \downarrow}\right), \\
\hat{H}_{\mathrm{m}}= & \hat{H}_{\mathrm{m}, \mathrm{loc}}-B \sum_{n=0}^{(L-1) / 2}\left[\hat{C}_{n, \uparrow}^{+} \hat{C}_{n, \uparrow}-\hat{C}_{n, \downarrow}^{+} \hat{C}_{n, \downarrow}\right]
\end{aligned}
$$

and $\hat{H}_{\mathrm{m}, \mathrm{loc}}=-B\left(\hat{d}_{\uparrow}^{+} \hat{d}_{\uparrow}-\hat{d}_{\downarrow}^{+} \hat{d}_{\downarrow}\right)$. Note that the $C$-parity eigenbasis in this one-dimensional finite-size chain takes on the role of the $s$-wave scattering in the three-dimensional continuous model [5], where all other spherical harmonics drop out of the problem.

Now, the impurity is at the left end of the half-chain. The half-chain has an odd number of sites $L_{\mathrm{hc}}=(L+1) / 2$, so that the system of half-chain plus impurity has a total number of even sites because we choose $(L+3) / 2$ to be even. In the absence of an external magnetic field, we have an equal number of electrons with spin $\sigma=\uparrow, \downarrow$ in the half-chain, $N_{\sigma}=(L+3) / 4$, so that the ground state has total spin zero.

\section{b. Kinetic energy of the half-chain}

We denote for $m=1, \ldots,(L+1) / 2$

$$
\begin{aligned}
\hat{b}_{m, \sigma} & \equiv(-1)^{m-1} \hat{a}_{2 m-1, \sigma}, \\
\epsilon(m) & =-2 t \cos \left[\frac{\pi(2 m-1)}{L+1}\right]
\end{aligned}
$$

and find

$$
\hat{T}^{C}=\sum_{m=1, \sigma}^{(L+1) / 2} \epsilon(m) \hat{b}_{m, \sigma}^{+} \hat{b}_{m, \sigma}
$$

for the kinetic energy of the half-chain in diagonal form. For half-filling of the half-chain, the Fermi energy is at $E_{\mathrm{F}}=0$, i.e., the last occupied site in Fourier space is $m_{\mathrm{F}}=(L+3) / 4$ with energy $\epsilon\left(m_{\mathrm{F}}\right)=0$.

We rewrite the dispersion relation as

$$
\epsilon(m)=2 t \sin \left[\frac{2 \pi\left(m-m_{\mathrm{F}}\right)}{L+1}\right],
$$

which can be linearized around the Fermi wave number

$$
\epsilon(m) \approx 2 t \frac{2 \pi\left(m-m_{\mathrm{F}}\right)}{L+1}+\mathcal{O}\left(\left(m-m_{\mathrm{F}}\right)^{3}\right)
$$

for $\left|m-m_{\mathrm{F}}\right| \ll L$ so that the Fermi velocity becomes

$$
v_{\mathrm{F}}=\frac{\epsilon\left(m_{\mathrm{F}}+1\right)-\epsilon\left(m_{\mathrm{F}}\right)}{2 \pi /(L+1)}=2 t .
$$

In Bethe-ansatz calculations, $v_{\mathrm{F}}^{\mathrm{BA}} \equiv 1$ is used. Therefore, it is convenient to set $2 t \equiv 1$ so that the bandwidth is $W=2$.

\section{Weak-coupling perturbation theory \\ a. Perturbation theory to leading order}

To leading order, we choose finite system sizes $L$, and consider the full-chain geometry. In Fourier space, the kinetic 
energy operator and the Kondo coupling for the chain are given by

$$
\begin{aligned}
\hat{T}= & \sum_{k=1, \sigma}^{L} \epsilon_{k} \hat{a}_{k, \sigma}^{+} \hat{a}_{k, \sigma}, \\
\hat{V}_{\mathrm{sd}}= & \frac{J_{\mathrm{K}}}{L+1} \sum_{k, p=1}^{L} \sin (\pi k / 2) \sin (\pi p / 2) \\
& \times\left(\hat{d}_{\downarrow}^{+} \hat{d}_{\uparrow} \hat{a}_{k, \uparrow}^{+} \hat{a}_{p, \downarrow}+\hat{d}_{\uparrow}^{+} \hat{d}_{\downarrow} \hat{a}_{k, \downarrow}^{+} \hat{a}_{p, \uparrow}\right. \\
& \left.+\frac{1}{2}\left(\hat{d}_{\uparrow}^{+} \hat{d}_{\uparrow}-\hat{d}_{\downarrow}^{+} \hat{d}_{\downarrow}\right)\left(\hat{a}_{k, \uparrow}^{+} \hat{a}_{p, \uparrow}-\hat{a}_{k, \downarrow}^{+} \hat{a}_{p, \downarrow}\right)\right) .
\end{aligned}
$$

Due to spin symmetry we find

$$
\left\langle\Phi_{0}|\hat{T}| \Phi_{0}\right\rangle=\langle A|\hat{T}| A\rangle=E_{\mathrm{FS}}-\epsilon_{k_{\mathrm{F}}}=E_{\mathrm{FS}}
$$

because $\epsilon_{k_{\mathrm{F}}}=0$. Moreover,

$$
\begin{aligned}
\left\langle\Phi_{0}\left|\hat{V}_{\mathrm{sd}}\right| \Phi_{0}\right\rangle= & -\frac{3 J_{\mathrm{K}}}{2(L+1)} \sum_{k, p=1}^{L} \sin (\pi k / 2) \sin (\pi p / 2) \\
& \times\left\langle A\left|\hat{a}_{k, \uparrow}^{+} \hat{a}_{p, \uparrow}-\hat{a}_{k, \downarrow}^{+} \hat{a}_{p, \downarrow}\right| A\right\rangle \\
= & -\frac{3 J_{\mathrm{K}}}{2(L+1)}
\end{aligned}
$$

because $\sin ^{2}\left(\pi k_{\mathrm{F}} / 2\right)=1$. Thus, we obtain

$$
e_{0}^{(1)}\left(J_{\mathrm{K}}\right)=E_{0}^{(1)}\left(J_{\mathrm{K}}\right)-E_{\mathrm{FS}}=-\frac{3 J_{\mathrm{K}}}{2} \frac{1}{L+1} .
$$

In first order in $J_{K}$, the energy decrease is only of the order $1 / L$.

\section{b. Perturbation theory to second order}

To second order, we implicitly work in the thermodynamic limit $L \rightarrow \infty$. The second-order energy correction reads as

$$
e_{0}^{(2)}\left(J_{\mathrm{K}}\right)=\sum_{|m\rangle \neq\left|\Phi_{0}\right\rangle} \frac{\left|\left\langle m\left|\hat{V}_{\mathrm{sd}}\right| \Phi_{0}\right\rangle\right|^{2}}{E_{0}^{(0)}-E_{m}^{(0)}} .
$$

All states that can be reached from $\left|\Phi_{0}\right\rangle$ by an application of $\hat{V}_{\text {sd }}$ have an extra particle in one of the $L / 2$ single-particle levels above the Fermi sea at $p>k_{\mathrm{F}}$ and a hole in one of the $L / 2$ single-particle levels below $k_{\mathrm{F}}, k \leqslant k_{\mathrm{F}}$ [see Eq. (A18)]. Therefore, $E_{0}^{(0)}-E_{k, p}^{(0)}=-\left(\epsilon_{p}-\epsilon_{k}\right)$. Otherwise, the coupling matrix element is independent of $k$ and $p$. Therefore, in the thermodynamic limit, we may sum over all $(L / 2)^{2}$ intermediate states and find

$$
e_{0}^{(2)}\left(J_{\mathrm{K}}\right)=-\left(\frac{2}{L}\right)^{2} \sum_{k=1}^{k_{\mathrm{F}}} \sum_{p=k_{\mathrm{F}}}^{L} \frac{\left\langle\Phi_{0}\left|\hat{V}_{\mathrm{sd}}^{2}\right| \Phi_{0}\right\rangle}{\epsilon_{p}-\epsilon_{k}} \equiv-f b_{1}^{2}
$$

with

$$
\begin{aligned}
f & =4 \int_{0}^{1} d \omega_{1} \int_{-1}^{0} d \omega_{2} \rho_{0}\left(\omega_{1}\right) \rho_{0}\left(\omega_{2}\right) \frac{1}{\omega_{1}-\omega_{2}}, \\
b_{1}^{2} & =\left\langle\Phi_{0}\left|\hat{V}_{\mathrm{sd}}^{2}\right| \Phi_{0}\right\rangle .
\end{aligned}
$$

As shown in Appendix B 2, we have $b_{1}^{2}=3 J_{\mathrm{K}}^{2} / 32$, independent of the density of states.
For the one-dimensional density of states (8) we find

$$
f=\int_{0}^{\infty} d \omega e^{-\omega \eta} J_{0}(\omega) \boldsymbol{H}_{0}(\omega)
$$

where $\eta=0^{+}, J_{0}(\omega)$ is the zeroth-order Bessel function, and $\boldsymbol{H}_{0}(\omega)$ is the zeroth-order Struve function [see Eqs. (9.1.18) and (12.1.7) of Ref. [36]]. Using Eq. (12.1.19) of Ref. [36] this can further be simplified to

$$
f=\frac{8}{\pi} \sum_{k=0}^{\infty} \frac{1}{2 k+1} \int_{0}^{\infty} d \omega J_{0}(\omega) J_{2 k+1}(\omega)=1,
$$

where we used Eq. (6.512,2) of Ref. [37]. Thus, our final result to second order is

$$
e_{0}^{(2)}\left(J_{\mathrm{K}}\right)=-\frac{3}{32} J_{\mathrm{K}}^{2}
$$

for the one-dimensional density of states (8).

\section{Energy of the magnetic Yosida state}

\section{a. Host-electron Fermi sea}

At half band filling and for a symmetric density of states we have

$$
|\mathrm{FS}\rangle=\prod_{k ; \epsilon_{k} \leqslant-\epsilon_{\mathrm{F}}} \hat{a}_{k, \downarrow}^{+} \prod_{k ; \epsilon_{k} \leqslant \epsilon_{\mathrm{F}}} \hat{a}_{k, \uparrow}^{+}|\mathrm{vac}\rangle .
$$

The host-electron spin polarization per site

$$
s_{0}=\frac{1}{2} \frac{1}{L} \sum_{k}\left\langle\mathrm{FS}\left|\hat{a}_{k, \uparrow}^{+} \hat{a}_{k, \uparrow}-\hat{a}_{k, \downarrow}^{+} \hat{a}_{k, \downarrow}\right| \mathrm{FS}\right\rangle
$$

determines $\epsilon_{\mathrm{F}}$. For the one-dimensional density of states we find

$$
s_{0}=\frac{1}{\pi} \arcsin \epsilon_{\mathrm{F}}, \quad \epsilon_{\mathrm{F}}=\sin \left(\pi s_{0}\right) .
$$

In turn, $\epsilon_{\mathrm{F}}$ can be viewed as a variational parameter that optimizes the ground-state energy in the presence of the external field

$$
\begin{aligned}
e_{\mathrm{FS}}\left(\epsilon_{\mathrm{F}}\right)=t_{\mathrm{FS}}\left(\epsilon_{\mathrm{F}}\right)-2 B s_{0}\left(\epsilon_{\mathrm{F}}\right), \\
t_{\mathrm{FS}}\left(\epsilon_{\mathrm{F}}\right)=\frac{1}{L}\langle\mathrm{FS}|\hat{T}| \mathrm{FS}\rangle \\
=2 \int_{-1}^{0} d \omega \omega \rho_{0}(\omega)+2 \int_{0}^{\epsilon_{\mathrm{F}}} d \omega \omega \rho_{0}(\omega) .
\end{aligned}
$$

For a symmetric density of states at half band filling, the variational optimum is at

$$
\epsilon_{\mathrm{F}}^{(0)}=B
$$

for $B \leqslant 1$ and $\epsilon_{\mathrm{F}}^{(0)}=1$ for $B \geqslant 1$. Thus, in one dimension for $B \leqslant 1$

$$
e_{\mathrm{FS}}(B)=-\frac{2}{\pi} \sqrt{1-B^{2}}-\frac{2}{\pi} B \arcsin (B) .
$$

\section{b. Presence of the impurity}

In the presence of the impurity, we have to determine $\epsilon_{\mathrm{F}}$ from the minimization of

$$
E_{0}\left(\epsilon_{\mathrm{F}}\right)=L e_{\mathrm{FS}}\left(\epsilon_{\mathrm{F}}\right)+e_{0}\left(\epsilon_{\mathrm{F}}\right) .
$$


This gives the condition

$$
0=L e_{\mathrm{FS}}^{\prime}\left(\epsilon_{\mathrm{F}}^{\mathrm{opt}}\right)+e_{0}^{\prime}\left(\epsilon_{\mathrm{F}}^{\mathrm{opt}}\right) .
$$

We separate the terms of order $L$ and order unity,

$$
\epsilon_{\mathrm{F}}^{\mathrm{opt}}=B+\frac{1}{L} \epsilon_{\mathrm{F}}^{(1)}
$$

where we use Eq. (A34) to identify the leading-order term $\epsilon_{\mathrm{F}}^{(0)}=B$, and find in Eq. (A37) that

$$
\begin{aligned}
0 & =e_{\mathrm{FS}}^{\prime \prime}(B) \epsilon_{\mathrm{F}}^{(1)}+e_{0}^{\prime}(B), \\
\epsilon_{\mathrm{F}}^{(1)} & =-\frac{e_{0}^{\prime}(B)}{e_{\mathrm{FS}}^{\prime \prime}(B)}=-\left.\frac{1}{2 \rho_{0}(B)} \frac{\partial e_{0}\left(\epsilon_{\mathrm{F}}\right)}{\partial \epsilon_{\mathrm{F}}}\right|_{\epsilon_{\mathrm{F}}=B} .
\end{aligned}
$$

In the last equation we used Eq. (A33) to show that

$$
\begin{aligned}
& e_{\mathrm{FS}}^{\prime}\left(\epsilon_{\mathrm{F}}\right)=2\left(\epsilon_{\mathrm{F}}-B\right) \rho_{0}\left(\epsilon_{\mathrm{F}}\right), \\
& e_{\mathrm{FS}}^{\prime \prime}\left(\epsilon_{\mathrm{F}}\right)=2 \rho_{0}\left(\epsilon_{\mathrm{F}}\right)+2\left(\epsilon_{\mathrm{F}}-B\right) \rho_{0}^{\prime}\left(\epsilon_{\mathrm{F}}\right) .
\end{aligned}
$$

Fortunately, we do not need to know $\epsilon_{\mathrm{F}}^{(1)}$ when we work with the ground-state energy because

$$
\begin{aligned}
E_{0}\left(\epsilon_{\mathrm{F}}^{\mathrm{opt}}\right) & =L\left(e_{\mathrm{FS}}(B)+e_{\mathrm{FS}}^{\prime}(B) \frac{\epsilon_{\mathrm{F}}^{(1)}}{L}\right)+e_{0}(B) \\
& =L e_{\mathrm{FS}}(B)+e_{0}(B)
\end{aligned}
$$

is independent of $\epsilon_{\mathrm{F}}^{(1)}$ due to Eq. (A33) [see also Eq. (A40)].

\section{c. Lagrange functional and minimization}

After calculating all expectation values, the Lagrange functional for the Yosida state $\mathcal{L} \equiv \mathcal{L}\left[\left\{\alpha_{k, \uparrow}\right\},\left\{\alpha_{k, \downarrow}\right\}, \lambda\right]$ becomes

$$
\begin{aligned}
\mathcal{L}= & \frac{1}{2}\left(\frac{1}{L} \sum_{k}^{\prime} \alpha_{k, \downarrow}^{2} \epsilon_{k}+\frac{1}{L} \sum_{k}^{\prime \prime} \alpha_{k, \uparrow}^{2} \epsilon_{k}\right) \\
& -\frac{J_{\mathrm{K}}}{2}\left(\frac{1}{L} \sum_{k}^{\prime} \alpha_{k, \downarrow}\right)\left(\frac{1}{L} \sum_{k}^{\prime \prime} \alpha_{k, \uparrow}\right) \\
& +\frac{J_{\mathrm{K}}}{4} s_{0}\left(\frac{1}{L} \sum_{k}^{\prime} \alpha_{k, \downarrow}^{2}-\frac{1}{L} \sum_{k}^{\prime \prime} \alpha_{k, \uparrow}^{2}\right) \\
& -\frac{J_{\mathrm{K}}}{8}\left(\frac{1}{L} \sum_{k}^{\prime} \alpha_{k, \downarrow}\right)^{2}-\frac{J_{\mathrm{K}}}{8}\left(\frac{1}{L} \sum_{k}^{\prime \prime} \alpha_{k, \uparrow}\right)^{2} \\
& +\lambda\left(1-\frac{1}{2 L} \sum_{k}^{\prime} \alpha_{k, \downarrow}^{2}-\frac{1}{2 L} \sum_{k}^{\prime \prime} \alpha_{k, \uparrow}^{2}\right),
\end{aligned}
$$

where we took the normalization into account using the Lagrange parameter $\lambda$.

We define

$$
\begin{aligned}
& C_{\uparrow}=\frac{1}{L} \sum_{k}^{\prime \prime} \alpha_{k, \uparrow}, \\
& C_{\downarrow}=\frac{1}{L} \sum_{k}^{\prime} \alpha_{k, \downarrow} .
\end{aligned}
$$

The variation of the Lagrange functional (A42) gives

$$
\begin{aligned}
& \alpha_{k, \uparrow}=\frac{J_{\mathrm{K}}}{4} \frac{C_{\uparrow}+2 C_{\downarrow}}{\epsilon_{k}-\left(\lambda+J_{\mathrm{K}} s_{0} / 2\right)}, \\
& \alpha_{k, \downarrow}=\frac{J_{\mathrm{K}}}{4} \frac{C_{\downarrow}+2 C_{\uparrow}}{\epsilon_{k}-\left(\lambda-J_{\mathrm{K}} s_{0} / 2\right)}
\end{aligned}
$$

in the respective regions in $k$ space. We abbreviate the principal-value integral

$$
F_{1}(x, B)=f_{B}^{1} d \omega \rho_{0}(\omega) \frac{1}{\omega-x}
$$

to find in Eq. (A43)

$$
\begin{aligned}
& C_{\uparrow}=\frac{J_{\mathrm{K}}}{4}\left(C_{\uparrow}+2 C_{\downarrow}\right) F_{1}\left(\lambda+J_{\mathrm{K}} s_{0} / 2, B\right), \\
& C_{\downarrow}=\frac{J_{\mathrm{K}}}{4}\left(C_{\downarrow}+2 C_{\uparrow}\right) F_{1}\left(\lambda-J_{\mathrm{K}} s_{0} / 2,-B\right)
\end{aligned}
$$

whereby we assume throughout that $0 \leqslant B<1$, i.e., the host electrons are not fully polarized. Note that Eq. (A41) permits to set $\epsilon_{\mathrm{F}}=B$ in our further considerations.

The secular determinant that belongs to Eq. (A46) must be zero because the normalization condition $\left(C_{\uparrow}+C_{\downarrow}\right) / 2=$ 1 must also be fulfilled. Therefore, we determine $\lambda$ from the equation

$$
\left(1-\frac{J_{\mathrm{K}} F_{+}}{4}\right)\left(1-\frac{J_{\mathrm{K}} F_{-}}{4}\right)-\frac{J_{\mathrm{K}}^{2} F_{+} F_{-}}{4}=0,
$$

where we abbreviated $F_{+} \equiv F_{1}\left(\lambda+J_{\mathrm{K}} s_{0} / 2, B\right)$ and $F_{-} \equiv$ $F_{1}\left(\lambda-J_{\mathrm{K}} s_{0} / 2,-B\right)$. In one spatial dimension we have $s_{0}(B)=(1 / \pi) \arcsin (B)$ from Eq. (A34) and

$$
F_{1}(x, B)=\frac{1}{\pi \sqrt{1-x^{2}}} \ln \left[\frac{1-B x+\sqrt{\left(1-B^{2}\right)\left(1-x^{2}\right)}}{B-x}\right] .
$$

Using the variationally optimal parameters, it is not difficult to show that

$$
e_{0}^{\mathrm{Y}}\left(J_{\mathrm{K}}, B\right)=\lambda .
$$

Therefore, Eq. (A47) determines the variational ground-state energy as a function of the external field $B$.

Equation (A47) provides a solution only for $B \leqslant B_{\mathrm{c}}^{\mathrm{Y}}\left(J_{\mathrm{K}}\right)$ above which the Yosida state becomes unstable. This problem does not occur in the Gutzwiller description so that we do not extend the Yosida state to the region $B>B_{\mathrm{c}}^{\mathrm{Y}}\left(J_{\mathrm{K}}\right)$.

\section{Impurity spin polarization and magnetization of the Yosida state \\ a. Impurity spin polarization}

By definition [see Eq. (14)], the impurity spin polarization is given by

$$
\frac{m^{S, \mathrm{Y}}\left(J_{\mathrm{K}}, B\right)}{g_{\mathrm{e}} \mu_{\mathrm{B}}}=S^{z, \mathrm{Y}}\left(J_{\mathrm{K}}, B\right)=\frac{1}{2}\left\langle\Psi_{\mathrm{Y}}\left|\hat{n}_{d, \uparrow}-\hat{n}_{d, \downarrow}\right| \Psi_{\mathrm{Y}}\right\rangle .
$$

This expectation value is readily calculated to give

$$
S^{z, \mathrm{Y}}\left(J_{\mathrm{K}}, B\right)=\frac{1}{2} \frac{1-3 J_{\mathrm{K}} F_{+} / 4}{1+J_{\mathrm{K}} F_{+} / 4}
$$


with $F_{+} \equiv F_{1}\left(e_{0}^{\mathrm{Y}}\left(J_{\mathrm{K}}, B\right)+J_{\mathrm{K}} s_{0}(B) / 2, B\right)$ and $F_{1}(x, B)$ from Eq. (57) in one dimension. The ground-state energy $e_{0}^{\mathrm{Y}}\left(J_{\mathrm{K}}, B\right)$ in the presence of a global field is derived from the solution of Eq. (56), and $s_{0}(B)=\arcsin (B) / \pi$ in one dimension.

When the external field is applied only locally, the impurity spin polarization is given by

$$
S_{\mathrm{loc}}^{z, \mathrm{Y}}\left(J_{\mathrm{K}}, B\right)=\frac{1}{2} \frac{1-3 J_{\mathrm{K}} F\left(e_{0, \mathrm{loc}}^{\mathrm{Y}}\left(J_{\mathrm{K}}, B\right)-B\right) / 4}{1+J_{\mathrm{K}} F\left(e_{0, \mathrm{loc}}^{\mathrm{Y}}\left(J_{\mathrm{K}}, B\right)-B\right) / 4}
$$

with $F(x)$ from Eq. (58) in one dimension. The groundstate energy $e_{0, \text { loc }}^{\mathrm{Y}}\left(J_{\mathrm{K}}, B\right)$ in the presence of a local field is derived from the solution of Eq. (56) when $F_{ \pm}$is replaced by $F(\lambda \mp B)$.

\section{b. Impurity-induced magnetization}

The solution $e_{0}^{\mathrm{Y}}\left(J_{\mathrm{K}}, B\right)$ of Eq. (56) must be determined numerically for given Kondo coupling and external field. The impurity-induced magnetization is then obtained using Eq. (20) as

$$
\frac{m^{\mathrm{ii}, \mathrm{Y}}\left(J_{\mathrm{K}}, B\right)}{g_{e} \mu_{\mathrm{B}}}=-\frac{1}{2} \frac{\partial e_{0}^{\mathrm{Y}}\left(J_{\mathrm{K}}, B\right)}{\partial B}
$$

by a numerical derivative of the Yosida ground-state energy in the presence of a magnetic field. Equation (A53) holds because of the variational Hellmann-Feynman theorem $[26,27]$ (see Appendix B 1).

To obtain an analytic expression, we take the derivative of Eq. (56) with respect to the magnetic field $B$. With the abbreviations

$$
\begin{aligned}
& s_{0}^{\prime}(B)=\frac{\partial s_{0}(B)}{\partial B}=\rho_{0}(B), \\
& y(B)=\frac{\rho_{0}(B)}{e_{0}^{\mathrm{Y}}\left(J_{\mathrm{K}}, B\right)+J_{\mathrm{K}} s_{0}(B) / 2-B},
\end{aligned}
$$

and

$$
\begin{aligned}
F_{2}(x, B) & =\frac{\partial F_{1}(x, B)}{\partial x}, \\
F_{2,+} & =F_{2}\left(e_{0}^{\mathrm{Y}}\left(J_{\mathrm{K}}, B\right)+J_{\mathrm{K}} s_{0}(B) / 2, B\right), \\
F_{2,-} & =F_{2}\left(e_{0}^{\mathrm{Y}}\left(J_{\mathrm{K}}, B\right)-J_{\mathrm{K}} s_{0}(B) / 2,-B\right)
\end{aligned}
$$

we find

$$
F_{1,+}^{\prime}+F_{1,-}^{\prime}+\frac{3 J_{\mathrm{K}}}{4}\left(F_{1,+}^{\prime} F_{1,-}+F_{1,-}^{\prime} F_{1,+}\right)=0,
$$

where

$$
\begin{aligned}
& F_{1,+}^{\prime}=y(B)+\left(-2 m^{\mathrm{ii}, \mathrm{Y}}(B)+\frac{J_{\mathrm{K}} \rho_{0}(B)}{2}\right) F_{2,+}, \\
& F_{1,-}^{\prime}=-y(-B)+\left(-2 m^{\mathrm{ii}, \mathrm{Y}}(B)-\frac{J_{\mathrm{K}} \rho_{0}(B)}{2}\right) F_{2,-} .
\end{aligned}
$$

Here, we used Eq. (A53), $e_{0}^{\mathrm{Y}}\left(J_{\mathrm{K}},-B\right)=e_{0}^{\mathrm{Y}}\left(J_{\mathrm{K}}, B\right)$, and $s_{0}(-B)=-s_{0}(B)$.

We solve Eq. (A56) for $m^{\mathrm{ii}, \mathrm{Y}}\left(J_{\mathrm{K}}, B\right)$,

$$
\frac{m^{\mathrm{ii}, \mathrm{Y}}\left(J_{\mathrm{K}}, B\right)}{g_{e} \mu_{\mathrm{B}}}=\frac{1}{4} \frac{N_{a}\left(J_{\mathrm{K}}, B\right)}{N_{b}\left(J_{\mathrm{K}}, B\right)}
$$

with

$$
\begin{aligned}
N_{a}\left(J_{\mathrm{K}}, B\right)= & 8 y(B)-8 y(-B) \\
& +4 J_{\mathrm{K}} \rho_{0}(B)\left(F_{2,+}-F_{2,-}\right) \\
& +6 J_{\mathrm{K}}\left(y(B) F_{1,-}-y(-B) F_{1,+}\right) \\
& +3 J_{\mathrm{K}}^{2} \rho_{0}(B)\left(F_{1,-} F_{2,+}-F_{1,+} F_{2,-}\right),
\end{aligned}
$$

and

$$
\begin{aligned}
N_{b}\left(J_{\mathrm{K}}, B\right)= & 4 F_{2,+}+4 F_{2,-} \\
& +3 J_{\mathrm{K}}\left(F_{1,-} F_{2,+}+F_{1,+} F_{2,-}\right) .
\end{aligned}
$$

When the external field is applied only locally, Eq. (56) reduces to

$$
\left(1-\frac{J_{\mathrm{K}} F_{+}}{4}\right)\left(1-\frac{J_{\mathrm{K}} F_{-}}{4}\right)-\frac{J_{\mathrm{K}}^{2} F_{+} F_{-}}{4}=0
$$

with $F_{ \pm}=F(\lambda \mp B)$ from Eq. (58). Then, Eq. (A58) reduces to

$$
m_{\mathrm{loc}}^{\mathrm{ii}, \mathrm{Y}}\left(J_{\mathrm{K}}, B\right)=\frac{4\left(F_{+}^{\prime}-F_{-}^{\prime}\right)+3 J_{\mathrm{K}}\left(F_{+}^{\prime} F_{-}-F_{-}^{\prime} F_{+}\right)}{8\left(F_{+}^{\prime}+F_{-}^{\prime}\right)+6 J_{\mathrm{K}}\left(F_{+}^{\prime} F_{-}+F_{-}^{\prime} F_{+}\right)}
$$

with

$$
\begin{aligned}
F_{ \pm}^{\prime} & =F^{\prime}(\lambda \pm B), \\
F^{\prime}(x) & =-\frac{1}{\pi x\left(1-x^{2}\right)}+\frac{x}{1-x^{2}} F(x) .
\end{aligned}
$$

In Fig. 21 we display the impurity spin polarization $S^{z, \mathrm{Y}}=m^{S, \mathrm{Y}} /\left(g_{e} \mu_{\mathrm{B}}\right)$ and the impurity-induced magnetization $m^{\mathrm{ii}, \mathrm{Y}} /\left(g_{e} \mu_{\mathrm{B}}\right)$ from the Yosida wave function as a function of global and local magnetic fields. The curves noticeably differ from each other which shows that it is important to distinguish between the four quantities.

For small interactions, the system has a large impurityinduced magnetic susceptibility so that small fields tend to fully polarize the system. Indeed, as seen from the figure, the impurity-induced magnetization reaches its maximum value for fields of the order of $10^{-2} \mathcal{D}$ at $J_{\mathrm{K}}=0.5$. For these small fields, it is not important whether the field is applied globally or only locally because the magnetic response is mostly determined by the impurity spin and by the electrons in its vicinity. Note that the impurity spin polarization $S^{z, Y}$ is smaller than the impurity-induced magnetization $m^{\mathrm{ii}, \mathrm{Y}} /\left(g_{e} \mu_{\mathrm{B}}\right)$; this is an artifact of the Yosida wave function.

Large interactions, $J_{\mathrm{K}}=2$, require large fields to polarize the impurity system. Thus, the differences between the curves are more pronounced. Recall, however, that the Yosida wave function is not a good variational state for strong couplings so that the curves in Fig. 21 are not representative for the Kondo model.

\section{Evaluation of expectation values for the Gutzwiller wave function}

a. Norm, kinetic energy, and impurity spin polarization

The Gutzwiller state is normalized to unity,

$$
\left\langle\Psi_{\mathrm{G}} \mid \Psi_{\mathrm{G}}\right\rangle=\left\langle\Phi\left|1-x\left(\hat{n}_{\uparrow}^{d}-n_{\uparrow}^{d, 0}\right)\left(\hat{n}_{\downarrow}^{d}-n_{\downarrow}^{d, 0}\right)\right| \Phi\right\rangle=1
$$

because $|\Phi\rangle$ is a normalized single-particle product state and Eq. (80) holds. Likewise [19], the expectation value of the 

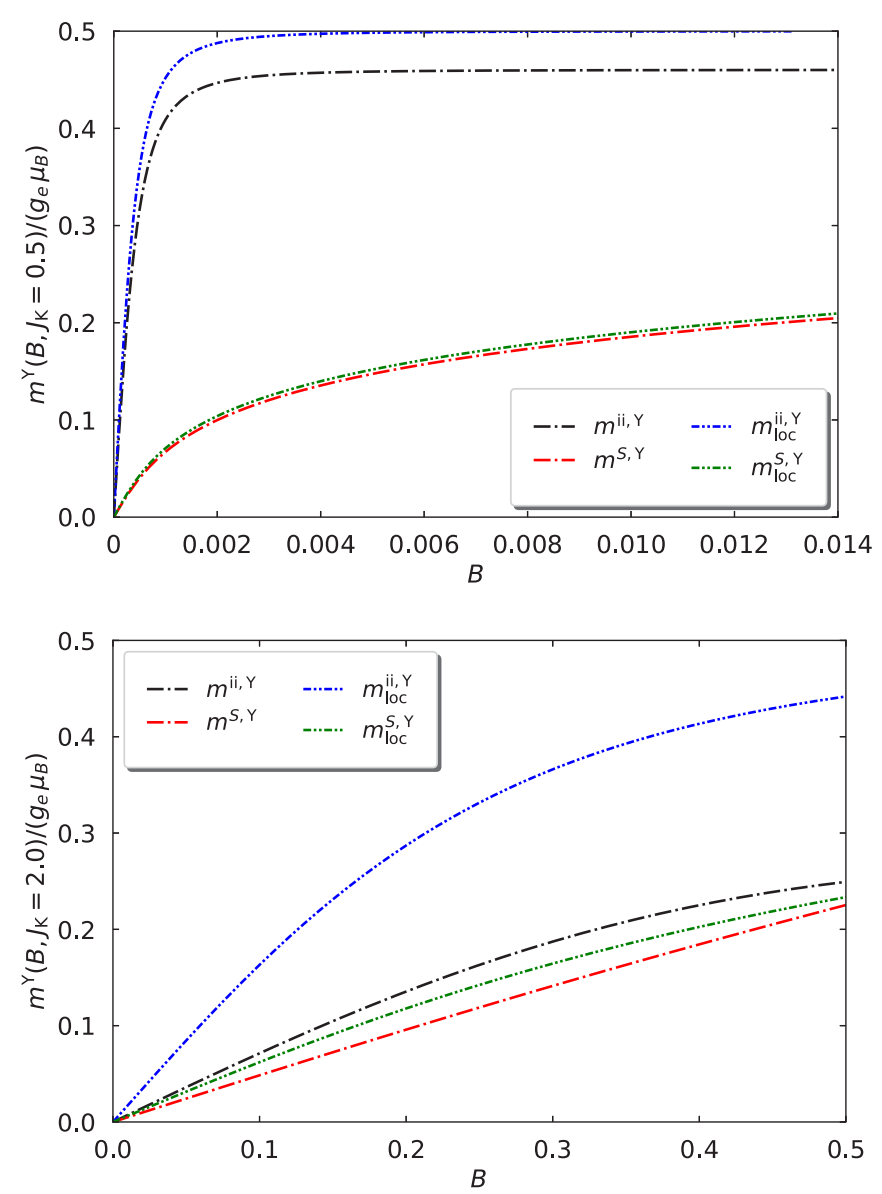

FIG. 21. Impurity spin polarization $S^{z, \mathrm{Y}}=m^{S, \mathrm{Y}} /\left(g_{e} \mu_{\mathrm{B}}\right)$ [Eq. (14)] and impurity-induced magnetization $m^{\mathrm{ii}, \mathrm{Y}} /\left(g_{e} \mu_{\mathrm{B}}\right)$ [Eq. (19)] of the one-dimensional symmetric Kondo model as a function of global/local fields for $J_{\mathrm{K}}=0.5$ (upper figure) and $J_{\mathrm{K}}=2$ (lower figure) from the Yosida wave function.

kinetic energy in the Gutzwiller wave function gives

$$
\begin{aligned}
\langle\hat{T}\rangle_{\mathrm{G}} & =\sum_{k, \sigma}\left[\epsilon(k)-\sigma_{n} B\right]\left\langle\Phi\left|\hat{a}_{k, \sigma}^{+} \hat{a}_{k, \sigma} \hat{P}_{\mathrm{G}}^{2}\right| \Phi\right\rangle \\
& =\sum_{k, \sigma}\left[\epsilon(k)-\sigma_{n} B\right]\left\langle\Phi\left|\hat{a}_{k, \sigma}^{+} \hat{a}_{k, \sigma}\right| \Phi\right\rangle,
\end{aligned}
$$

when we use Eqs. (77) and (80). Moreover, it is readily seen from Eq. (83) that

$$
S^{z, \mathrm{G}}=\frac{1}{2}\left\langle\hat{n}_{\uparrow}^{d}-\hat{n}_{\downarrow}^{d}\right\rangle_{\mathrm{G}}=m=\frac{1}{2}\left\langle\Phi\left|\hat{n}_{\uparrow}^{d}-\hat{n}_{\downarrow}^{d}\right| \Phi\right\rangle
$$

so that we do not have to distinguish between the impurity spin polarization in $\left|\Psi_{\mathrm{G}}\right\rangle$ and in $|\Phi\rangle$.

\section{b. Spin-flip terms in the Kondo coupling}

Using Eq. (83) we have

$$
\left\langle\hat{V}_{\mathrm{sd}}^{\mathrm{sf}}\right\rangle_{\mathrm{G}}=\lambda_{\uparrow} \lambda_{\downarrow} \frac{J_{\mathrm{K}}}{2 L} \sum_{k, k^{\prime}}\langle\Phi|\left[\hat{a}_{k^{\prime}, \uparrow}^{+} \hat{a}_{k, \downarrow} \hat{d}_{\downarrow}^{+} \hat{d}_{\uparrow}+\text { H.c. }\right]|\Phi\rangle .
$$

We use Wick's theorem for the single-particle product state $|\Phi\rangle$ to find

$$
\begin{aligned}
\left\langle\hat{V}_{\mathrm{sd}}^{\mathrm{sf}}\right\rangle_{\mathrm{G}}= & -\frac{2}{\sqrt{1-4 m^{2}}} \frac{J_{\mathrm{K}}}{2 L} \\
& \times\left(\sum_{k}\left\langle\Phi\left|\hat{d}_{\downarrow}^{+} \hat{a}_{k, \downarrow}\right| \Phi\right\rangle \sum_{k^{\prime}}\left\langle\Phi\left|\hat{a}_{k^{\prime}, \uparrow}^{+} \hat{d}_{\uparrow}\right| \Phi\right\rangle\right. \\
& \left.+\sum_{k}\left\langle\Phi\left|\hat{d}_{\uparrow}^{+} \hat{a}_{k, \uparrow}\right| \Phi\right\rangle \sum_{k^{\prime}}\left\langle\Phi\left|\hat{a}_{k^{\prime}, \downarrow}^{+} \hat{d}_{\downarrow}\right| \Phi\right\rangle\right) .
\end{aligned}
$$

\section{c. Ising terms in the Kondo coupling}

Using Eq. (83) we have

$$
\begin{aligned}
\left\langle\hat{V}_{\mathrm{sd}}^{\mathrm{Is}}\right\rangle_{\mathrm{G}}= & \frac{J_{\mathrm{K}}}{4 L} \sum_{k, k^{\prime}}\langle\Phi|\left(\hat{a}_{k^{\prime}, \uparrow}^{+} \hat{a}_{k, \uparrow}-\hat{a}_{k^{\prime}, \downarrow}^{+} \hat{a}_{k, \downarrow}\right) \\
& \times\left(\frac{\hat{d}_{\uparrow}^{+} \hat{d}_{\uparrow}}{n_{\uparrow}^{d, 0}}-\frac{\hat{d}_{\downarrow}^{+} \hat{d}_{\downarrow}}{n_{\downarrow}^{d, 0}}+2 m \frac{\hat{n}_{\uparrow}^{d} \hat{n}_{\downarrow}^{d}}{n_{\uparrow}^{d, 0} n_{\downarrow}^{d, 0}}\right)|\Phi\rangle \\
= & -\frac{J_{\mathrm{K}}}{2 L}\left(\sum_{k}\left\langle\Phi\left|\hat{d}_{\uparrow}^{+} \hat{a}_{k, \uparrow}\right| \Phi\right\rangle \sum_{k^{\prime}}\left\langle\Phi\left|\hat{a}_{k^{\prime}, \uparrow}^{+} \hat{d}_{\uparrow}\right| \Phi\right\rangle\right. \\
& \left.+\sum_{k}\left\langle\Phi\left|\hat{d}_{\downarrow}^{+} \hat{a}_{k, \downarrow}\right| \Phi\right\rangle \sum_{k^{\prime}}\left\langle\Phi\left|\hat{a}_{k^{\prime}, \downarrow}^{+} \hat{d}_{\downarrow}\right| \Phi\right\rangle\right) \\
& +J_{\mathrm{K}} m M_{0},
\end{aligned}
$$

where we again used Wick's theorem for the single-particle product state $|\Phi\rangle$, and defined the host-electron spin polarization on the impurity

$$
M_{0}=\frac{1}{2 L} \sum_{k, k^{\prime}}\left\langle\Phi\left|\hat{a}_{k^{\prime}, \uparrow}^{+} \hat{a}_{k, \uparrow}-\hat{a}_{k^{\prime}, \downarrow}^{+} \hat{a}_{k, \downarrow}\right| \Phi\right\rangle .
$$

\section{Lagrange functional and effective noninteracting SIAM for the Gutzwiller wave function}

\section{a. Optimization of the Lagrange functional}

We must optimize $\langle\hat{H}\rangle_{\mathrm{G}}$ with respect to the single-particle product states $|\Phi\rangle$ that are normalized to unity, $\langle\Phi \mid \Phi\rangle=1$. Moreover, we must respect the conditions (78) and (A69).

We apply the Ritz variational principle to the Lagrange functional $\mathcal{L} \equiv \mathcal{L}\left(\{|\Phi\rangle\}, E_{\text {sp }}, E_{d}, K\right)$ (see also Ref. [19]):

$$
\begin{aligned}
\mathcal{L}= & \langle\hat{H}\rangle_{\mathrm{G}}+E_{\mathrm{sp}}(1-\langle\Phi \mid \Phi\rangle) \\
& +E_{d}\left(2 m-\left\langle\Phi\left|\hat{n}_{\uparrow}^{d}-\hat{n}_{\downarrow}^{d}\right| \Phi\right\rangle\right) \\
& -K\left(2 M_{0}-\frac{1}{L} \sum_{k, k^{\prime}}\left\langle\Phi\left|\hat{a}_{k^{\prime}, \uparrow}^{+} \hat{a}_{k, \uparrow}-\hat{a}_{k^{\prime}, \downarrow}^{+} \hat{a}_{k, \downarrow}\right| \Phi\right\rangle\right), \\
\langle\hat{H}\rangle_{\mathrm{G}}= & \langle\hat{T}\rangle_{\mathrm{G}}-2 m B+\left\langle\hat{V}_{\mathrm{sd}}^{\mathrm{sf}}\right\rangle_{\mathrm{G}}+\left\langle\hat{V}_{\mathrm{sd}}^{\mathrm{Is}}\right\rangle_{\mathrm{G}},
\end{aligned}
$$

and find that $|\Phi\rangle$ must obey the Schrödinger equation

$$
\hat{H}_{0}^{\mathrm{SIAM}}|\Phi\rangle=E_{\mathrm{sp}}|\Phi\rangle
$$


with the effective noninteracting single-impurity Anderson model (SIAM)

$$
\begin{aligned}
\hat{H}_{0}^{\text {SIAM }}= & \widetilde{T}+\widetilde{V}-E_{d}\left(\hat{n}_{\uparrow}^{d}-\hat{n}_{\downarrow}^{d}\right) \\
& +\frac{K}{L} \sum_{k, k^{\prime}}\left(\hat{a}_{k^{\prime}, \uparrow}^{+} \hat{a}_{k, \uparrow}-\hat{a}_{k^{\prime}, \downarrow}^{+} \hat{a}_{k, \downarrow}\right),
\end{aligned}
$$

with the operators for the kinetic energy and the local hybridization

$$
\begin{aligned}
& \widetilde{T}=\sum_{k, \sigma}\left[\epsilon(k)-\sigma_{n} B\right] \hat{a}_{k, \sigma}^{+} \hat{a}_{k, \sigma}, \\
& \widetilde{V}=\frac{1}{\sqrt{L}} \sum_{k, \sigma} V_{\sigma}\left(\hat{a}_{k, \sigma}^{+} \hat{d}_{\sigma}+\hat{d}_{\sigma}^{+} \hat{a}_{k, \sigma}\right),
\end{aligned}
$$

where

$$
\begin{aligned}
& V_{\sigma}=-\frac{J_{\mathrm{K}}}{2}\left(\gamma_{\sigma}+\frac{2}{\sqrt{1-4 m^{2}}} \gamma_{\bar{\sigma}}\right), \\
& \gamma_{\sigma}=\sqrt{\frac{1}{L}} \sum_{k}\left\langle\Phi\left|\hat{a}_{k, \sigma}^{+} \hat{d}_{\sigma}\right| \Phi\right\rangle
\end{aligned}
$$

have to be determined self-consistently. Hereby, we assumed that $\gamma_{\sigma}$ and $V_{\sigma}$ are real; recall $\uparrow=\downarrow, \bar{\downarrow}=\uparrow$.

In the following we choose $|\Phi\rangle$ as the ground-state of $\hat{H}_{0}^{\text {SIAM }}$ in Eq. (A71) and denote the optimal single-particle product state by $\left|\Phi_{0}\right\rangle$. Recall that we have to also fulfill the conditions (78) and (A69),

$$
\begin{aligned}
m & =\frac{1}{2}\left\langle\Phi_{0}\left|\hat{n}_{\uparrow}^{d}-\hat{n}_{\downarrow}^{d}\right| \Phi_{0}\right\rangle, \\
M_{0} & =\frac{1}{2 L} \sum_{k, k^{\prime}}\left\langle\Phi_{0}\left|\hat{a}_{k^{\prime}, \uparrow}^{+} \hat{a}_{k, \uparrow}-\hat{a}_{k^{\prime}, \downarrow}^{+} \hat{a}_{k, \downarrow}\right| \Phi_{0}\right\rangle,
\end{aligned}
$$

which we regain from the minimization of $\mathcal{L}$ with respect to $E_{d}$ and $K$. The minimization of $\mathcal{L}$ with respect to $m$ and $M_{0}$ gives

$$
\begin{aligned}
K & =\frac{1}{2} J_{\mathrm{K}} m, \\
E_{d} & =B-\frac{1}{2} J_{\mathrm{K}} M_{0}+\frac{4 m}{\left(1-4 m^{2}\right)^{3 / 2}} J_{\mathrm{K}} \gamma_{\uparrow} \gamma_{\downarrow} .
\end{aligned}
$$

Note that a finite impurity magnetization $m$ generates a potential scattering in the effective single-impurity Anderson model $K \neq 0$.

\section{b. Self-consistency procedure}

The remaining task is the calculation of the single-particle density of states for the effective noninteracting singleimpurity Anderson Hamiltonian (A71). Using the singleparticle density of states, we can calculate the single-particle energy $E_{\mathrm{sp}}\left(B, E_{d}, K, V_{\sigma}\right)$ from which we obtain $m, M_{0}$, and $\gamma_{\sigma}$ :

$$
\begin{aligned}
2 m & =-\frac{\partial E_{\mathrm{sp}}\left(B, E_{d}, K, V_{\sigma}\right)}{\partial E_{d}}, \\
2 M_{0} & =\frac{\partial E_{\mathrm{sp}}\left(B, E_{d}, K, V_{\sigma}\right)}{\partial K} \\
2 \gamma_{\sigma} & =\frac{\partial E_{\mathrm{sp}}\left(B, E_{d}, K, V_{\sigma}\right)}{\partial V_{\sigma}}
\end{aligned}
$$

when we use the Hellmann-Feynman theorem that also holds for variational approaches. The simple proof relies on the fact that the optimized variational state is stationary with respect to small wave-function variations (see Appendix B 1).

Therefore, the parameters of the single-impurity Anderson model are determined self-consistently using the following procedure.

S 1 The self-consistency procedure is initialized by choosing the values of the paramagnetic solution $m=0, M_{0}=0$, $K=0$, and $V_{\sigma}=V$ (see Sec. VIB). This guarantees that the algorithm works for $B=0$. To lift the degeneracy, we start at $E_{d}=B$ for given $B>0, J_{\mathrm{K}}>0$.

$\mathrm{S} 2$ The analytic expressions for $E_{\mathrm{sp}}\left(B, E_{d}, K, V_{\sigma}\right)$ give new values for $m, M_{0}, \gamma_{\sigma}$ from Eq. (A77), and thus new values for $K$ and $E_{d}$ from Eq. (A76), and new values for $V_{\sigma}$ from Eq. (A74).

S 3 Check whether or not $K, E_{d}, V_{\sigma}$ deviate from their previous values by more than some small value $\eta=10^{-12}$. If so, return to $\mathrm{S} 2$, otherwise, the algorithm terminates and gives the self-consistent values for $K, E_{d}, V_{\sigma}$ and $m, M_{0}, \gamma_{\sigma}$.

\section{c. Ground-state energy}

Note that $E_{\mathrm{sp}}$ is not identical to $E_{0}^{\mathrm{G}}=\left\langle\hat{H}_{\mathrm{G}}\right\rangle$. Instead, we have from Eq. (A73)

$$
\begin{aligned}
E_{\mathrm{sp}}= & \langle\widetilde{T}\rangle_{0}+\langle\widetilde{V}\rangle_{0}-2 m E_{d}+2 K M_{0} \\
= & \sum_{k, \sigma}\left[\epsilon(k)-\sigma_{n} B\right]\left\langle\Phi\left|\hat{a}_{k, \sigma}^{+} \hat{a}_{k, \sigma}\right| \Phi\right\rangle \\
& +\sum_{\sigma} 2 V_{\sigma} \gamma_{\sigma}-2 m E_{d}+2 K M_{0} \\
= & \sum_{k, \sigma}\left[\epsilon(k)-\sigma_{n} B\right]\left\langle\Phi\left|\hat{a}_{k, \sigma}^{+} \hat{a}_{k, \sigma}\right| \Phi\right\rangle-2 m E_{d}+2 K M_{0} \\
& -J_{\mathrm{K}}\left(\gamma_{\uparrow}^{2}+\gamma_{\downarrow}^{2}+\frac{4}{\sqrt{1-4 m^{2}}} \gamma_{\uparrow} \gamma_{\downarrow}\right),
\end{aligned}
$$

where we used Eq. (A74) to replace $V_{\sigma}$ by $\gamma_{\sigma}$. In contrast, from Eq. (A70) we have

$$
\begin{aligned}
E_{0}^{\mathrm{G}}= & \sum_{k, \sigma}\left[\epsilon(k)-\sigma_{n} B\right]\left\langle\Phi\left|\hat{a}_{k, \sigma}^{+} \hat{a}_{k, \sigma}\right| \Phi\right\rangle-m\left(2 B-J_{\mathrm{K}} M_{0}\right) \\
& -\frac{J_{\mathrm{K}}}{2}\left(\frac{4}{\sqrt{1-4 m^{2}}} \gamma_{\downarrow} \gamma_{\uparrow}+\gamma_{\uparrow}^{2}+\gamma_{\downarrow}^{2}\right) .
\end{aligned}
$$

Comparing both equations results in

$$
\begin{aligned}
E_{0}^{\mathrm{G}}= & E_{\mathrm{sp}}+\frac{J_{\mathrm{K}}}{2}\left(\frac{4}{\sqrt{1-4 m^{2}}} \gamma_{\downarrow} \gamma_{\uparrow}+\gamma_{\uparrow}^{2}+\gamma_{\downarrow}^{2}\right) \\
& -m\left(2 B-J_{\mathrm{K}} M_{0}\right)+2 m E_{d}-2 K M_{0} \\
= & E_{\mathrm{sp}}+\frac{J_{\mathrm{K}}}{2}\left(\frac{4}{\sqrt{1-4 m^{2}}} \gamma_{\downarrow} \gamma_{\uparrow}+\gamma_{\uparrow}^{2}+\gamma_{\downarrow}^{2}\right) \\
& +\frac{8 m^{2}}{\left(1-4 m^{2}\right)^{3 / 2}} J_{\mathrm{K}} \gamma_{\uparrow} \gamma_{\downarrow}-J_{\mathrm{K}} m M_{0}
\end{aligned}
$$

for the Gutzwiller variational ground-state energy of the Kondo model. The excess ground-state energy for the 
Gutzwiller variational state is given by

$$
\begin{aligned}
e_{0}^{\mathrm{G}}\left(J_{\mathrm{K}}, B\right)= & E_{0}^{\mathrm{G}}\left(J_{\mathrm{K}}, B\right) \\
& -\sum_{k, \sigma}\left(\epsilon(k)-\sigma_{n} B\right)\left\langle\mathrm{FS}\left|\hat{a}_{k, \sigma}^{+} \hat{a}_{k, \sigma}\right| \mathrm{FS}\right\rangle,
\end{aligned}
$$

where $|\mathrm{FS}\rangle$ is the Fermi sea of noninteracting electrons.

The variational optimization for $m>0$ is outlined in Appendix B 4. Here, we summarize the main results.

(i) The Gutzwiller ground state displays a finite local magnetization $m>0$ at $B=0^{+}$for all $0<J_{\mathrm{K}}<J_{\mathrm{K}, \mathrm{c}}^{\mathrm{G}} \approx 0.839$. The precise value is determined in Appendix A $8 \mathrm{~g}$.

(ii) For small interactions $J_{\mathrm{K}} \rightarrow 0$, the values for $V_{\sigma}$, $\gamma_{\sigma}, E_{d}, K, m, M_{0}$, and $\omega_{p, \downarrow}$ can be determined analytically.

(iii) The ground-state energy for small interactions in one dimension can be approximated by

$$
e_{0}^{\mathrm{G}}\left(J_{\mathrm{K}}\right) \approx-0.0905 J_{\mathrm{K}}^{2}-0.051 J_{\mathrm{K}}^{3}-0.05 J_{\mathrm{K}}^{4}
$$

for the Gutzwiller variational energy for $J_{\mathrm{K}} \lesssim 0.4$. The quadratic coefficient can be compared with the exact result from perturbation theory $e_{0}\left(J_{\mathrm{K}}\right) \approx-3 J_{\mathrm{K}}^{2} / 32=-0.09375 J_{\mathrm{K}}^{2}$ [see Eq. (34)]. The magnetic Gutzwiller states account for $96.5 \%$ of the correlation energy.

\section{Zero-field impurity spin susceptibility for the paramagnetic Gutzwiller state}

From the numerical solution of the self-consistency equations, we see that $\gamma_{\uparrow}=\gamma_{\downarrow}$ and $V_{\uparrow}=V_{\downarrow}$ at self-consistency. In the following, we use this assumption.

\section{a. Impurity spin polarization}

The optimization procedure of Appendix A 7 directly gives the impurity spin polarization

$$
\frac{m^{S, \mathrm{G}}\left(J_{\mathrm{K}}, B\right)}{g_{e} \mu_{\mathrm{B}}}=m\left(J_{\mathrm{K}}, B\right) .
$$

When the external field is applied only at the impurity, we simply replace the expression $\left[\epsilon(k)-\sigma_{n} B\right]$ by $\epsilon(k)$ in Eqs. (A64), (A73), (A78), (A79), and (A81) to arrive at the corresponding "local" expressions for the impurity spin polarization and impurity-induced magnetization. Invoking the variational Hellmann-Feynman theorem [26,27] (see Appendix B 1), we may alternatively use

$$
\frac{m_{\mathrm{loc}}^{\mathrm{S}, \mathrm{G}}\left(J_{\mathrm{K}}, B\right)}{g_{e} \mu_{\mathrm{B}}}=-\frac{1}{2} \frac{\partial e_{0, \mathrm{loc}}^{\mathrm{G}}\left(J_{\mathrm{K}}, B\right)}{\partial B}
$$

[see also Eq. (22)].

\section{b. Impurity-induced magnetization}

Following the steps in Appendix A 6 it is readily shown that

$$
\frac{m^{\mathrm{ii}, \mathrm{G}}\left(J_{\mathrm{K}}, B\right)}{g_{e} \mu_{\mathrm{B}}}=\left\langle\Phi_{0}\left|\hat{S}^{z}+\hat{s}^{z}\right| \Phi_{0}\right\rangle-\left\langle\mathrm{FS}\left|\hat{s}^{z}\right| \mathrm{FS}\right\rangle .
$$

Here, $\left|\Phi_{0}\right\rangle$ is the optimized ground state of the effective noninteracting single-impurity Anderson model Hamiltonian defined in Eq. (A72) and $|\mathrm{FS}\rangle$ is the Fermi-sea ground state of noninteracting electrons in the presence of a magnetic field. When we use the single-particle density of states of the noninteracting SIAM (see Appendix B 4), we find

$$
\begin{aligned}
\frac{m^{\mathrm{ii}, \mathrm{G}}\left(J_{\mathrm{K}}, B\right)}{g_{e} \mu_{\mathrm{B}}}= & \frac{1}{2} \int_{-\infty}^{0} d \omega\left[D_{\uparrow}(\omega)-D_{\downarrow}(\omega)\right] \\
& -\frac{1}{2} \int_{-\infty}^{0} d \omega\left[D_{\uparrow}^{\mathrm{FS}}(\omega)-D_{\downarrow}^{\mathrm{FS}}(\omega)\right] \\
= & \frac{1}{2} \int_{-\infty} d \omega\left[D_{\mathrm{imp}, \uparrow}(\omega)-D_{\mathrm{imp}, \downarrow}(\omega)\right],
\end{aligned}
$$

where the impurity density of states is given by the phase-shift function

$$
\begin{aligned}
D_{\text {imp }, \sigma}(\omega) & =-\frac{1}{\pi} \frac{\partial \varphi_{\sigma}\left(\omega, B, E_{d}, K, V\right)}{\partial \omega}, \\
\cot \left[\varphi_{\sigma}\left(\omega, B, E_{d}, K, V\right)\right] & =\frac{\mathrm{R}_{\sigma}(\omega)}{\mathrm{I}_{\sigma}(\omega)},
\end{aligned}
$$

with the real and imaginary parts of the hybridization function

$$
\begin{aligned}
\mathrm{R}_{\sigma}(\omega)= & \left(\omega+\sigma_{n} E_{d}\right)\left[1-\sigma_{n} K \Lambda_{0}\left(\omega+\sigma_{n} B\right)\right] \\
& -V^{2} \Lambda_{0}\left(\omega+\sigma_{n} B\right), \\
\mathrm{I}_{\sigma}(\omega)= & \eta\left[1-\sigma_{n} K \Lambda_{0}\left(\omega+\sigma_{n} B\right)\right] \\
& +\left[\left(\omega+\sigma_{n} E_{d}\right) \sigma_{n} K+V^{2}\right] \pi \rho_{0}\left(\omega+\sigma_{n} B\right)
\end{aligned}
$$

[see Eqs. (IV-43) and (IV-44) of the Supplemental Material [23] and $\eta=0^{+}$.

Since the impurity contribution to the density of states is given by a frequency derivative, the frequency integration in Eq. (A86) is readily carried out. The density of states vanishes for $\omega \rightarrow-\infty$ so that the density of states at the Fermi energy alone determines the impurity-induced magnetization. We focus on the paramagnetic region for the Gutzwiller wave function $J_{\mathrm{K}}>J_{\mathrm{K}, \mathrm{c}}^{\mathrm{G}}$, so that the band part of the impurity density of states at the Fermi energy gives [see Eq. (IV-50) of the Supplemental Material [23]]

$$
\begin{aligned}
\frac{m^{\mathrm{ii}, \mathrm{G}}\left(J_{\mathrm{K}}, B\right)}{g_{e} \mu_{\mathrm{B}}}= & -\frac{1}{2 \pi}\left[X_{\uparrow}(0)-X_{\downarrow}(0)\right], \\
X_{\sigma}(\omega) \equiv & X_{\sigma}\left(\omega, B, E_{d}, K, V\right) \\
= & \pi \theta_{\mathrm{H}}\left(-\omega-\sigma_{n} E_{d}\right) \\
& +\operatorname{arccot}\left[\frac{\left(\omega+\sigma_{n} E_{d}\right) \sqrt{1-\left(\omega+\sigma_{n} B\right)^{2}}}{\left(\omega+\sigma_{n} E_{d}\right) \sigma_{n} K+V^{2}}\right]
\end{aligned}
$$

in one dimension. Thus, we obtain the final result

$$
\begin{aligned}
\frac{m^{\mathrm{ii}, \mathrm{G}}\left(J_{\mathrm{K}}, B\right)}{g_{e} \mu_{\mathrm{B}}} & =\frac{1}{2}-\frac{1}{\pi} \operatorname{arccot}\left[\frac{E_{d} \sqrt{1-B^{2}}}{E_{d} K+V^{2}}\right] \\
& =\frac{1}{\pi} \arctan \left[\frac{E_{d} \sqrt{1-B^{2}}}{E_{d} K+V^{2}}\right],
\end{aligned}
$$

where $E_{d}(B), K(B)$, and $V(B)$ are determined from the solution of the self-consistency cycle in Appendix A 7 .

When the field is only applied locally, the same considerations lead to

$$
\frac{m_{\mathrm{loc}}^{\mathrm{ii}, \mathrm{G}}\left(J_{\mathrm{K}}, B\right)}{g_{e} \mu_{\mathrm{B}}}=\frac{1}{\pi} \arctan \left[\frac{E_{d, \mathrm{loc}}}{E_{d, \mathrm{loc}} K_{\mathrm{loc}}+V_{\mathrm{loc}}^{2}}\right],
$$




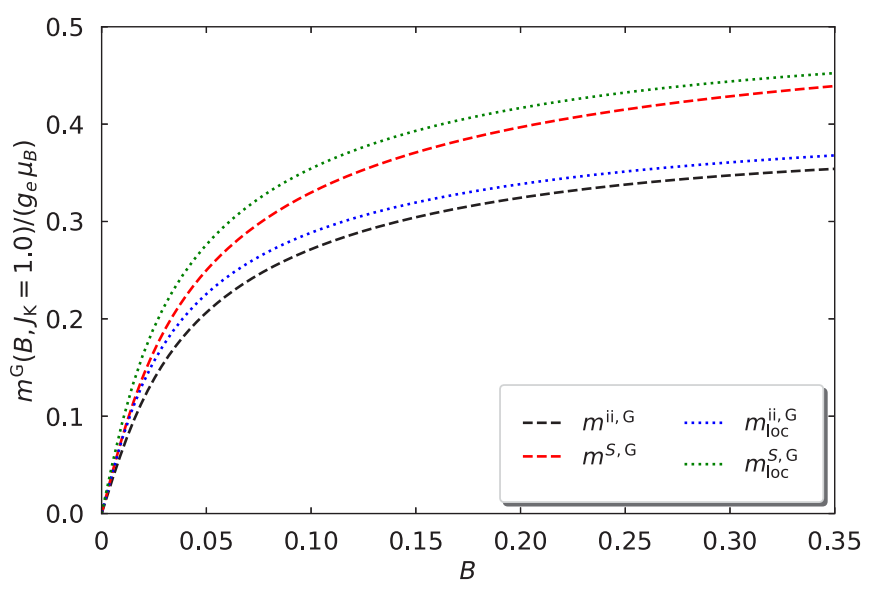

FIG. 22. Impurity spin polarization $S^{z, \mathrm{G}}=m^{S, \mathrm{G}} /\left(g_{e} \mu_{\mathrm{B}}\right)$ [Eqs. (A83) and (A84)] and impurity-induced magnetization $m^{\mathrm{ii}, \mathrm{G}} /\left(g_{e} \mu_{\mathrm{B}}\right)$ [Eqs. (A90) and (A91)] of the one-dimensional symmetric Kondo model as a function of global/local fields for $J_{\mathrm{K}}=1$ from the Gutzwiller wave function.

where the self-consistency problem has to be solved for a local field only. We show the impurity spin polarization and the impurity-induced magnetization as a function of an applied global/local magnetic field in Fig. 22 for $J_{\mathrm{K}}=1$, where the Gutzwiller wave function describes a local spin singlet.

In contrast to the Yosida wave function, the Gutzwiller wave function correctly shows that the impurity spin polarization is larger than the impurity-induced magnetization because the impurity spin is surrounded by a cloud of conduction electrons that screens the impurity spin. As in the Yosida wave function, the impurity spin polarization does not depend much on whether the magnetic field is applied globally or locally.

\section{c. Small fields}

In the paramagnetic phase $J_{\mathrm{K}}>J_{\mathrm{K}, \mathrm{c}}^{\mathrm{G}}$, and for small fields $B \rightarrow 0$, we can derive explicit results for the zero-field impurity spin susceptibility because it is sufficient to solve the self-consistency equations to linear order in the external field.

Keeping all terms up to linear order in $B$, we make the ansatz

$$
\begin{aligned}
\omega_{p, \uparrow} & =\omega_{p}+\bar{\omega}_{p} B, \quad \omega_{p, \downarrow}=\omega_{p}-\bar{\omega}_{p} B, \\
K & =\bar{K} B, \quad E_{d}=\bar{E}_{d} B, \\
M_{0} & =\bar{M}_{0} B, \quad m=2 \chi B,
\end{aligned}
$$

where $\chi$ is the desired zero-field impurity-spin susceptibility in units of $\left(g_{e} \mu_{\mathrm{B}}\right)^{2}$ :

$$
\frac{\chi_{0}^{S, \mathrm{G}}\left(J_{\mathrm{K}}, B\right)}{\left(g_{e} \mu_{\mathrm{B}}\right)^{2}}=\chi .
$$

In one dimension at $B=0$, the pole is at $\omega_{p}=-v_{+}$[see Eq. (85)]. Moreover, from Eqs. (21) and (A90) we find

$$
\frac{\chi_{0}^{\mathrm{ii}, \mathrm{G}}\left(J_{\mathrm{K}}\right)}{\left(g_{e} \mu_{\mathrm{B}}\right)^{2}}=\frac{\bar{E}_{d}}{2 \pi V^{2}}
$$

where $V_{\uparrow}=V_{\downarrow}=V$ and $\gamma_{\uparrow}=\gamma_{\downarrow}=\gamma=-2 V /\left(3 J_{\mathrm{K}}\right)$, with corrections of the order $B^{2}$, and with

$$
J_{\mathrm{K}}(V)=-\frac{8 V}{3}\left(\frac{\partial e_{0}(V)}{\partial V}\right)^{-1}
$$

where $V$ instead of $J_{\mathrm{K}}$ parametrizes the strength of the Kondo interaction. For $e_{0}(V)$, see Eq. (84).

Apparently, we have five unknowns, namely,

$$
\underline{v}^{\mathrm{T}}=\left(\bar{\omega}_{p}, \bar{E}_{d}, \bar{K}, \bar{M}_{0}, \chi\right),
$$

and we need five independent linear equations that connect these quantities.

\section{d. Useful integrals}

For later use we define the following set of integrals:

$$
J_{n}(V)=\int_{-1}^{0} \frac{d \omega}{\pi} \frac{\omega^{n} \sqrt{1-\omega^{2}}}{\left(\omega^{2}-\omega^{4}+V^{4}\right)^{2}} .
$$

Using Mathematica [29], the required integrals read as

$$
\begin{aligned}
J_{1}(V)= & -\frac{1}{2 \pi V^{4}\left(1+4 V^{4}\right)} \\
& +\frac{\left(-2+\sqrt{1+4 V^{4}}\right) \arctan \left(1 / v_{-}\right)}{2 \pi v_{-}\left(1+4 V^{4}\right)^{3 / 2}} \\
& +\frac{\left(2+\sqrt{1+4 V^{4}}\right)}{4 \pi v_{+}\left(1+4 V^{4}\right)^{3 / 2}} \ln \left(\frac{v_{+}-1}{v_{+}+1}\right)
\end{aligned}
$$

and

$$
\begin{aligned}
J_{3}(V)= & \frac{1}{\pi\left(1+4 V^{4}\right)} \\
& +\frac{\left(-3-4 V^{4}+\sqrt{1+4 V^{4}}\right) \arctan \left(1 / v_{-}\right)}{4 \pi v_{-}\left(1+4 V^{4}\right)^{3 / 2}} \\
& +\frac{\left(3+4 V^{4}+\sqrt{1+4 V^{4}}\right)}{8 \pi v_{+}\left(1+4 V^{4}\right)^{3 / 2}} \ln \left(\frac{v_{+}-1}{v_{+}+1}\right) .
\end{aligned}
$$

For $v_{ \pm}$, see Eq. (85).

\section{e. Five equations}

As shown in Appendix B 4,

$$
\bar{E}_{d}=1-\frac{J_{\mathrm{K}}}{2} \bar{M}_{0}+8 J_{\mathrm{K}} \chi \gamma^{2}
$$

with $\gamma=-2 V /\left(3 J_{\mathrm{K}}\right)$,

$$
\begin{gathered}
\bar{K}=J_{\mathrm{K}} \chi \\
\bar{E}_{d}\left(\omega_{p}^{2}-1\right)+\left(2 \omega_{p}^{2}-1\right) \bar{\omega}_{p}+\omega_{p}^{2}-\bar{K} V^{2}=0, \\
\bar{M}_{0}=\bar{M}_{0}^{\mathrm{b}}+\bar{M}_{0}^{\mathrm{band}}, \\
\bar{M}_{0}^{\mathrm{b}}=\frac{\bar{K}\left(-\omega_{p}^{2}+2 \omega_{p}^{4}+V^{4}\right)-\omega_{p}^{2}\left(3+\bar{E}_{d}+4 \bar{\omega}_{p}\right) V^{2}}{\omega_{p}\left(1-2 \omega_{p}^{2}\right)^{2}}, \\
\bar{M}_{0}^{\text {band }}=-2 \bar{K} V^{2} J_{3}(V)+2\left(\bar{E}_{d}-1\right) V^{4} J_{1}(V), \\
\chi=\chi^{\mathrm{b}}+\chi^{\mathrm{band}}, \\
\chi^{\mathrm{b}}=\frac{\omega_{p}\left(1+\bar{E}_{d}+\omega_{p}^{2}-\bar{E}_{d} \omega_{p}^{2}+2 \bar{\omega}_{p}-\bar{K} V^{2}\right)}{2\left(1-2 \omega_{p}^{2}\right)^{2}} \\
\chi^{\text {band }}=\frac{1}{2 \pi V^{2}}-V^{2}\left\{\left(\bar{E}_{d}-1\right)\left[J_{1}(V)-J_{3}(V)\right]\right. \\
\left.+\bar{K} V^{2} J_{1}(V)\right\} .
\end{gathered}
$$


Equations (A100)-(A104) are the required five equations for the five unknowns in Eq. (A96).

\section{f. Matrix problem}

The resulting matrix problem reads as, with $\omega_{p}=-v_{+}$[see Eq. (85)] and with $\underline{v}$ [from Eq. (A96)]

$$
\underline{\underline{M}} \cdot \underline{v}=\underline{g} .
$$

Here, the matrix has the form

$$
\underline{\underline{M}}=\left(\begin{array}{ccccc}
0 & 1 & 0 & J_{\mathrm{K}} / 2 & -8 J_{\mathrm{K}} \gamma^{2} \\
0 & 0 & 1 & 0 & -J_{\mathrm{K}} \\
2 \omega_{p}^{2}-1 & \omega_{p}^{2}-1 & -V^{2} & 0 & 0 \\
M_{41} & M_{42} & M_{43} & -1 & 0 \\
M_{51} & M_{52} & M_{53} & 0 & -1
\end{array}\right)
$$

with the nonzero matrix elements

$$
\begin{aligned}
M_{41} & =-\frac{4 \omega_{p} V^{2}}{\left(1-2 \omega_{p}^{2}\right)^{2}} \\
M_{42} & =-\frac{\omega_{p} V^{2}}{\left(1-2 \omega_{p}^{2}\right)^{2}}+2 V^{4} J_{1}(V) \\
M_{43} & =\frac{\left(-\omega_{p}^{2}+2 \omega_{p}^{4}+V^{4}\right)}{\omega_{p}\left(1-2 \omega_{p}^{2}\right)^{2}}-2 V^{2} J_{3}(V), \\
M_{51} & =\frac{\omega_{p}}{\left(1-2 \omega_{p}^{2}\right)^{2}}, \\
M_{52} & =\frac{\omega_{p}\left(1-\omega_{p}^{2}\right)}{2\left(1-2 \omega_{p}^{2}\right)^{2}}-V^{2}\left[J_{1}(V)-J_{3}(V)\right] \\
M_{53} & =-\frac{V^{2} \omega_{p}}{2\left(1-2 \omega_{p}^{2}\right)^{2}}-V^{4} J_{1}(V) .
\end{aligned}
$$

For a global external field, the inhomogeneity reads as

$$
\underline{g}^{\mathrm{T}}=\left(1,0,-\omega_{p}^{2}, g_{4}, g_{5}\right)
$$

where

$$
\begin{aligned}
g_{4}= & \frac{3 \omega_{p} V^{2}}{\left(1-2 \omega_{p}^{2}\right)^{2}}+2 V^{4} J_{1}(V), \\
g_{5}= & -\frac{\omega_{p}\left(1+\omega_{p}^{2}\right)}{2\left(1-2 \omega_{p}^{2}\right)^{2}} \\
& -V^{2}\left[J_{1}(V)-J_{3}(V)\right]-\frac{1}{2 \pi V^{2}} .
\end{aligned}
$$

When the external field is applied only locally, the matrix $\underline{\underline{M}}$ and the vector $\underline{v}$ in Eq. (A105) remain unchanged but we have for the inhomogeneity

$$
\underline{g}_{\mathrm{loc}}^{\mathrm{T}}=(1,0,0,0,0) .
$$

The matrix problem (A105) can be solved analytically, generating large expressions. Eventually, we solve it numerically.

\section{g. Strong-coupling limit}

For the nontrivial entries in the matrix $\underline{\underline{M}}$ we have

$$
\begin{aligned}
& M_{14}=\frac{2 V}{3}+\frac{1}{6 V}-\frac{4}{9 \pi V^{2}}+\frac{5}{48 V^{3}} \\
& M_{15}=-\frac{8 V}{3}+\frac{2}{3 V}-\frac{16}{9 \pi V^{2}}+\frac{1}{4 V^{3}} \\
& M_{25}=-\frac{4 V}{3}-\frac{1}{3 V}+\frac{8}{9 \pi V^{2}}-\frac{5}{24 V^{3}}, \\
& M_{31}=2 V^{2}+\frac{1}{4 V^{2}}, \\
& M_{32}=V^{2}-\frac{1}{2}+\frac{1}{8 V^{2}} \\
& M_{33}=-V^{2}, \\
& M_{41}=\frac{1}{V}+\frac{1}{4 V^{3}}, \\
& M_{42}=\frac{1}{4 V}+\frac{1}{16 V^{3}},
\end{aligned}
$$

and

$$
\begin{aligned}
& M_{43}=-\frac{3}{4 V}-\frac{1}{16 V^{3}} \\
& M_{51}=-\frac{1}{4 V^{3}} \\
& M_{52}=\frac{1}{8 V}-\frac{1}{32 V^{3}}, \\
& M_{53}=\frac{1}{8 V}+\frac{1}{32 V^{3}},
\end{aligned}
$$

up to and including order $1 / V^{3}$. To the same order,

$$
\begin{gathered}
\underline{g}^{\mathrm{T}}=\left(1,0,-V^{2}-1 / 2-1 /\left(8 V^{2}\right),-3 /(4 V)-3 /\left(16 V^{3}\right),\right. \\
\left.1 /(8 V)-1 /\left(2 \pi V^{2}\right)+7 /\left(32 V^{3}\right)\right)^{\mathrm{T}} .
\end{gathered}
$$

Lastly, for $J_{\mathrm{K}} \gg 1$ we have from Eq. (93)

$$
V\left(J_{\mathrm{K}}\right)=\frac{3}{4} J_{\mathrm{K}}-\frac{1}{3 J_{\mathrm{K}}}+\frac{32}{27 \pi J_{\mathrm{K}}^{2}}-\frac{14}{27 J_{\mathrm{K}}^{3}}+\mathcal{O}\left(1 / J_{\mathrm{K}}^{4}\right)
$$

Then, Mathematica [29] gives the vector $\underline{v}$,

$$
\begin{aligned}
\underline{v}^{\mathrm{T}}= & \left(\bar{\omega}_{p}, \bar{E}_{d}, \bar{K}, \bar{M}_{0}, \chi\right) \\
= & \left(-1-\frac{4}{3 \pi V}+\frac{29}{18 \pi V^{3}}, 1+\frac{13}{3 \pi V}-\frac{7}{36 \pi V^{3}},\right. \\
& \left.\frac{5}{3 \pi V}+\frac{31}{36 \pi V^{3}},-\frac{3}{2 \pi V^{2}}, \frac{5}{4 \pi V^{2}}\right)^{\mathrm{T}},
\end{aligned}
$$

up to and including order $1 / V^{3}$. Thus, in the strong-coupling limit, the impurity spin susceptibility in the Gutzwiller wave function is given by

$$
\frac{\chi_{0}^{S, \mathrm{G}}\left(J_{\mathrm{K}} \gg 1\right)}{\left(g_{e} \mu_{\mathrm{B}}\right)^{2}}=\frac{5}{4 \pi V^{2}}+\mathcal{O}\left(1 / V^{4}\right)=\frac{20}{9 \pi J_{\mathrm{K}}^{2}}+\mathcal{O}\left(1 / J_{\mathrm{K}}^{4}\right) .
$$


For a local external field, we obtain in the strong-coupling limit

$$
\begin{aligned}
\underline{v}_{\mathrm{loc}}^{\mathrm{T}}= & \left(-1+\frac{7}{12 V^{2}}-\frac{1}{3 \pi V^{3}}, \frac{5}{2}+\frac{2}{3 V^{2}}+\frac{1}{3 \pi V^{3}},\right. \\
& \frac{1}{2}+\frac{7}{12 V^{2}}-\frac{1}{3 \pi V^{3}},-\frac{3}{4 V}+\frac{3}{16 V^{3}}, \\
& \left.\frac{3}{8 V}+\frac{11}{32 V^{3}}\right)^{\mathrm{T}},
\end{aligned}
$$

up to and including order $1 / V^{3}$. Thus, for a local magnetic field, the strong-coupling limit of the impurity spin susceptibility in the Gutzwiller wave function is given by

$$
\frac{\chi_{0, \mathrm{loc}}^{S, \mathrm{G}}\left(J_{\mathrm{K}} \gg 1\right)}{\left(g_{e} \mu_{\mathrm{B}}\right)^{2}}=\frac{3}{8 V}+\frac{11}{32 V^{3}}=\frac{1}{2 J_{\mathrm{K}}}+\frac{28}{27 J_{\mathrm{K}}^{3}}+\mathcal{O}\left(1 / J_{\mathrm{K}}^{4}\right)
$$

For the zero-field impurity-induced susceptibilities in Eq. (A94), we find in the strong-coupling limit

$$
\begin{aligned}
\frac{\chi_{0}^{\mathrm{ii}, \mathrm{G}}\left(J_{\mathrm{K}} \gg 1\right)}{\left(g_{e} \mu_{\mathrm{B}}\right)^{2}} & =\frac{1}{2 \pi V^{2}}\left[1+\frac{13}{3 \pi V}-\frac{7}{36 \pi V^{3}}+\mathcal{O}\left(V^{-4}\right)\right] \\
& =\frac{8}{9 \pi J_{\mathrm{K}}^{2}}+\frac{416}{81 \pi^{2} J_{\mathrm{K}}^{3}}+\mathcal{O}\left(1 / J_{\mathrm{K}}^{4}\right)
\end{aligned}
$$

and

$$
\begin{aligned}
\frac{\chi_{0, \mathrm{loc}}^{\mathrm{ii}, \mathrm{G}}\left(J_{\mathrm{K}} \gg 1\right)}{\left(g_{e} \mu_{\mathrm{B}}\right)^{2}} & =\frac{1}{2 \pi V^{2}}\left[\frac{5}{2}+\frac{2}{3 V^{2}}+\frac{1}{3 \pi V^{3}}+\mathcal{O}\left(V^{-4}\right)\right] \\
& =\frac{20}{9 \pi J_{\mathrm{K}}^{2}}+\mathcal{O}\left(1 / J_{\mathrm{K}}^{4}\right) .
\end{aligned}
$$

The impurity spin susceptibility for a local field goes to zero proportional to $1 / J_{\mathrm{K}}$; all other susceptibilities vanish proportional to $J_{\mathrm{K}}^{-2}$. From Eqs. (A116) and (A120) we see that $\chi_{0}^{S, \mathrm{G}}\left(J_{\mathrm{K}}\right)$ and $\chi_{0, \mathrm{lic}}^{\mathrm{ii}, \mathrm{G}}\left(J_{\mathrm{K}}\right)$ agree to order $J_{\mathrm{K}}^{-2}$. A closed inspection shows that the expressions indeed agree to order $J_{\mathrm{K}}^{-4}$.

\section{Hamiltonian of the Wilson chain}

The matrix elements are calculated recursively from the starting values

$$
\begin{aligned}
\varepsilon_{0, \sigma} & =\int_{-1-\sigma_{n} B}^{1-\sigma_{n} B} d \epsilon \epsilon \rho_{0}\left(\epsilon+\sigma_{n} B\right), \\
t_{0, \sigma}^{2} & =\sum_{m}\left[\left(\zeta_{m, \sigma}^{+}-\varepsilon_{0, \sigma}\right)^{2}\left(\gamma_{m, \sigma}^{+}\right)^{2}+\left(\zeta_{m, \sigma}^{-}-\varepsilon_{0, \sigma}\right)^{2}\left(\gamma_{m, \sigma}^{-}\right)^{2}\right], \\
u_{0, m, \sigma} & =\gamma_{m, \sigma}^{+}, \\
v_{0, m, \sigma} & =\gamma_{m, \sigma}^{-}, \\
u_{1, m, \sigma} & =\frac{\left(\zeta_{m, \sigma}^{+}-\varepsilon_{0, \sigma}\right) u_{0, m, \sigma}}{t_{0, \sigma}}, \\
v_{1, m, \sigma} & =\frac{\left(\zeta_{m, \sigma}^{-}-\varepsilon_{0, \sigma}\right) v_{0, m, \sigma}}{t_{0, \sigma}} .
\end{aligned}
$$

For $n \geqslant 1$ one has to calculate iteratively

$$
\begin{aligned}
\varepsilon_{n, \sigma} & =\sum_{m} \zeta_{m, \sigma}^{+} u_{n, m, \sigma}^{2}+\zeta_{m, \sigma}^{-} u_{n, m, \sigma}^{2} \\
t_{n, \sigma}^{2} & =\sum_{m}\left[\left(\zeta_{m, \sigma}^{+}\right)^{2} u_{n, m, \sigma}^{2}+\left(\zeta_{m, \sigma}^{-}\right)^{2} v_{n, m, \sigma}^{2}\right]-t_{n-1, \sigma}^{2}-\varepsilon_{n, \sigma}^{2}
\end{aligned}
$$

and

$$
\begin{aligned}
u_{n+1, m, \sigma} & =\frac{\left(\zeta_{m, \sigma}^{+}-\varepsilon_{n, \sigma}\right) u_{n, m, \sigma}-t_{n-1, \sigma} u_{n-1, m, \sigma}}{t_{n, \sigma}} \\
v_{n+1, m, \sigma} & =\frac{\left(\zeta_{m, \sigma}^{-}-\varepsilon_{n, \sigma}\right) v_{n, m, \sigma}-t_{n-1, \sigma} v_{n-1, m, \sigma}}{t_{n, \sigma}}
\end{aligned}
$$

This concludes the derivation of the Wilson chain Hamiltonian.

\section{APPENDIX B: OUTLINE OF THE SUPPLEMENTAL MATERIAL}

In this Appendix, we summarize the content of the Supplemental Material [23].

\section{Hellmann-Feynman theorem}

We formulate and prove the Hellmann-Feynman theorem and its variational counterpart.

\section{Lanczos approach}

We recapitulate the Lanczos approach and show its variational property. Moreover, we calculate the first-order Lanczos coefficients for a constant and a one-dimensional density of states.

\section{Scattering off a local impurity}

We calculate the single-particle Green function for electrons that scatter off a local potential. The results permit the calculation of the ground-state energy of the Kondo model for large Kondo couplings.

\section{Noninteracting SIAM in the presence of potential scattering and a magnetic field}

We calculate the single-particle Green function for electrons in the noninteracting single-impurity Anderson model in the presence of a magnetic field and a local scattering potential. We use it to calculate the ground-state energy, the magnetization, and the zero-field susceptibility in the SIKM for the Gutzwiller wave function. We also discuss the magnetic transition in the Gutzwiller wave function.

\section{Ground-state energy from Bethe ansatz}

We collect the Bethe-ansatz equations and use them to derive the ground-state energy. Unfortunately, the Bethe ansatz does not provide tangible results for this quantity because the Bethe-ansatz energy is of third order in the Kondo coupling. 


\section{Free energy in second-order weak-coupling perturbation theory}

We rederive the expressions for the free energy to second order in the Kondo coupling at finite temperature and external magnetic field for a regular density of states of the host electrons. These results are used to calculate the Wilson number and the dominant term in the zero-field susceptibilities whereby we generalize previous results in the literature.
[1] W. J. de Haas, J. de Boer, and G. J. van dën Berg, Physica (Amsterdam) 1, 1115 (1934).

[2] J. Kondo, Prog. Theor. Phys. 32, 37 (1964).

[3] D. R. Hamann, Phys. Rev. 158, 570 (1967).

[4] A. Hewson, The Kondo Problem to Heavy Fermions (Cambridge University Press, Cambridge, 1993).

[5] K. G. Wilson, Rev. Mod. Phys. 47, 773 (1975).

[6] H. R. Krishna-murthy, J. W. Wilkins, and K. G. Wilson, Phys. Rev. B 21, 1003 (1980).

[7] H. R. Krishna-murthy, J. W. Wilkins, and K. G. Wilson, Phys. Rev. B 21, 1044 (1980).

[8] R. Bulla, T. A. Costi, and T. Pruschke, Rev. Mod. Phys. 80, 395 (2008).

[9] P. Nozières, J. Low Temp. Phys. 17, 31 (1974).

[10] A. Tsvelick and P. Wiegmann, Adv. Phys. 32, 453 (1983).

[11] N. Andrei, K. Furuya, and J. H. Lowenstein, Rev. Mod. Phys. 55, 331 (1983).

[12] J. D. Mancini and D. C. Mattis, Phys. Rev. B 31, 7440 (1985).

[13] M. Höck and J. Schnack, Phys. Rev. B 87, 184408 (2013).

[14] C. Lanczos, J. Res. Natl. Bur. Stand. 45, 255 (1950).

[15] E. Koch, in The LDA + DMFT Approach to Strongly Correlated Materials, Schriften des Forschungszentrums Jülich: Modeling and Simulation, Vol. 1, edited by E. Pavarini, E. Koch, A. Lichtenstein, and D. Vollhardt (Forschungszentrum Jülich, 2011), Chap. 8, p. 235.

[16] K. Yosida, Phys. Rev. 147, 223 (1966).

[17] K. Yosida, Theory of Magnetism, Solid-State Sciences Series, Vol. 122 (Springer, Berlin, Heidelberg, 1996).

[18] M. C. Gutzwiller, Phys. Rev. 134, A923 (1964).

[19] T. Linneweber, J. Bünemann, Z. Mahmoud, and F. Gebhard, J. Phys. Condens. Matter 29, 445603 (2017).
[20] S. R. White and R. M. Noack, Phys. Rev. Lett. 68, 3487 (1992).

[21] S. R. White, Phys. Rev. Lett. 69, 2863 (1992).

[22] S. R. White, Phys. Rev. B 48, 10345 (1993).

[23] See Supplemental Material at http://link.aps.org/supplemental/ 10.1103/PhysRevB.101.075132 for details of the calculations.

[24] J. R. Schrieffer and P. A. Wolff, Phys. Rev. 149, 491 (1966).

[25] J. Sólyom, Fundamentals of the Physics of Solids (Springer, Berlin, 2009), Vol. 3.

[26] H. Hellmann, Einführung in die Quantenchemie (Springer, Berlin, 2015).

[27] R. Feynman, Phys. Rev. 56, 340 (1939).

[28] G. Barcza, F. Gebhard, T. Linneweber, and Ö. Legeza, Phys. Rev. B 99, 165130 (2019).

[29] Wolfram Research, Inc., Mathematica, Version 11 (Champaign, IL, 2016).

[30] J. Bünemann, S. Wasner, E. von Oelsen, and G. Seibold, Philos. Mag. 95, 550 (2015).

[31] K. Yosida and A. Yoshimori, in Magnetic Properties of Metallic Alloys, Magnetism, Vol. V, edited by H. Suhl (Academic, New York, 1973), p. 253.

[32] M. Bortz and A. Klümper, J. Phys. A: Math. Gen. 37, 6413 (2004).

[33] Ö. Legeza, J. Röder, and B. A. Hess, Phys. Rev. B 67, 125114 (2003).

[34] Ö. Legeza and J. Sólyom, Phys. Rev. B 70, 205118 (2004).

[35] R. Hager, Diploma thesis, Universität Augsburg, 2007 (in German).

[36] M. Abramowitz and I. Stegun, Handbook of Mathematical Functions (Dover, New York, 1964).

[37] I. S. Gradshteyn and I. M. Ryzhik, Table of Integrals, Series, and Products, 7th ed. (Elsevier/Academic, Amsterdam, 2007) 